\title{
WELL-POSEDNESS THEORY FOR STOCHASTICALLY FORCED CONSERVATION LAWS ON RIEMANNIAN MANIFOLDS
}

\author{
LUCA GALIMBERTI AND KENNETH H. KARLSEN
}

\begin{abstract}
We investigate a class of scalar conservation laws on manifolds driven by multiplicative Gaussian (Itô) noise. The Cauchy problem defined on a Riemanian manifold is shown to be well-posed. We prove existence of generalized kinetic solutions using the vanishing viscosity method. A rigidity result àla Perthame is derived, which implies that generalized solutions are kinetic solutions and that kinetic solutions are uniquely determined by their initial data ( $L^{1}$ contraction principle). Deprived of noise, the equations we consider coincide with those analyzed by Ben-Artzi and LeFloch [7, who worked with Kružkov-DiPerna solutions. In the Euclidian case, the stochastic equations agree with those examined by Debussche and Vovelle [15].
\end{abstract}

\section{Contents}

1. Introduction

2. Background and hypotheses 5

2.1. Geometric framework 5

$\begin{array}{lll}2.2 . \quad \text { Stochastic framework } & 6\end{array}$

$3 . \quad$ Kinetic solutions and main result 8

4. Rigidity and uniqueness results 11

4.1. Localized equations 11

\begin{tabular}{lll}
\hline 4.2. & Regularization of localized equations & 12
\end{tabular}

4.3. Global equation and renormalization 24

\begin{tabular}{|ll|l|}
\hline 4.4. & Proof of Proposition & 4.1 \\
\hline
\end{tabular}

$\begin{array}{lll}4.5 . & \text { Uniqueness part of Theorem } 3.2 & 30\end{array}$

\begin{tabular}{lll}
\hline 4.6. & Proofs of technical lemmas & 31
\end{tabular}

\begin{tabular}{lll}
\hline 5. & Stochastic parabolic problem & 37
\end{tabular}

6. Generalized Itô formula 40

\begin{tabular}{ll}
\hline 7. Existence result & 49
\end{tabular}

7.1. Kinetic formulation of parabolic SPDE 49

7.2. A priori $L^{p}$ estimates 51

\begin{tabular}{lll}
\hline 7.3. & Bounds on kinetic measure & 57
\end{tabular}

\begin{tabular}{lll}
\hline 7.4. & Existence part of Theorem 3.2 & 58
\end{tabular}

$\begin{array}{lll}7 . \text { Appendix } & 58\end{array}$

\begin{tabular}{ll}
\hline References & 59
\end{tabular}

Date: June 27, 2019.

2010 Mathematics Subject Classification. Primary: 60H15, 35L65; Secondary: 58J, 35D30.

Key words and phrases. Stochastic conservation law, Riemannian manifold, kinetic solution.

This work was partially supported by the Research Council of Norway through the project Stochastic Conservation Laws (250674/F20). 


\section{INTRODUCTION}

Hyperbolic conservation laws constitute a significant class of nonlinear partial differential equations (PDEs) that arises in numerous applications. Indeed, the starting point of many mathematical models are balance equations for physical quantities such as mass, momentum, and energy. Prominent examples include the Euler and Saint-Venant (shallow water) equations. Many advances in fluid dynamics are built upon the mathematical theory of hyperbolic conservation laws, which was developed to answer questions regarding existence, uniqueness, and structure of weak solutions (shock waves). Most aspects of the theory of conservation laws are nicely summarized in the monumental book [11.

In recent years many researchers added new effects and features to conservation laws in order to account for additional (or more realistic) physical phenomena. One interesting situation arises when the domain of the solution to a hyperbolic PDE is a curved manifold, in which case the curvature of the domain alters the underlying dynamics. Significant applications include geophysical fluid dynamics, e.g. shallow water waves on the surface of a planet (caricature model of the atmosphere), and general relativity in which the Einstein-Euler equations are posed on a manifold with the metric being one of the unknowns. For scalar conservation laws defined on manifolds, the development of a theory of well-posedness and numerical approximations (of Kružkov-DiPerna solutions) was initiated by LeFloch and co-authors [1, 2, 6, 7, 8, 44, 45, 46] (see also Panov [52, 53]). The subject has been extended in several directions by different authors, including Giesselmann [30, Dziuk, Kröner, and Müller [24], Lengeler and Müller [47, Giesselmann and Müller [31, and Kröner, Müller, and Strehlau [40, and Graf, Kunzinger, and Mitrovic [32.

In a different direction, many researchers have made attempts to extend the scope of hyperbolic conservation laws (on Euclidean domains) by adding "random" effects. Randomness can enter these nonlinear PDEs in different ways, such as through stochastic forcing (source term) or in uncertain system parameters (flux function). Recently the mathematical study of stochastic conservation laws has emerged as an active field of study, linking several areas of mathematics, including nonlinear analysis and probability theory. Several works have studied the effect of Itô-type stochastic forcing on scalar conservation laws. With emphasis on questions related to existence and uniqueness of generalized solutions, we mention Kim 38 (see also Vallet and Wittbold [58]), who established the well-posedness of Kružkov solutions in the additive noise case. Feng and Nualart [27] presented a non-trivial modification of the Kružkov framework that ensured the well-posedness for nonlinear noise functions (multiplicative noise). Debussche and Vovelle 15 advanced a general existence and uniqueness theory based on kinetic solutions. Additional results can be found in Bauzet, Vallet, and Wittbold [5], Chen, Ding, and Karlsen [10], Hofmanová [35], Biswas, Karlsen, and Majee [9], Karlsen and Storrøsten [36], Debussche and Vovelle [16, Debussche, Hofmanová, and Vovelle [17, Lv and Wu 48, Kobayasi and Noboriguchi [39, and Dotti and Vovelle [22, 23]. A class of scalar conservation laws with "rough path" flux was introduced and analyzed by Lions, Perthame, and Souganidis in a series of works [49, 50, 51]. They developed a pathwise well-posedness theory based on kinetic solutions. This theory was further extended by Gess and Souganidis [28, 29].

No previous work has investigated the combined effect of nonlinear domains and Gaussian noise on the dynamics of shock waves. In this paper we are interested in the well-posedness of generalized solutions for a class of scalar conservation laws that are posed on a curved manifold and perturbed by a Gaussian Itô-type noise term. More precisely, let $(M, h)$ be an $n$-dimensional $(n \geq 1)$ smooth Riemannian manifold, which we assume is compact, connected, oriented, and with no boundary 
$(\partial M=\emptyset)$. We study the Cauchy problem for stochastically forced conservation laws of the form

$$
\begin{aligned}
& d u+\operatorname{div}_{h} f_{x}(u) d t=B(u) d W(t), \quad x \in M, 0<t<T, \\
& u(0, x)=u_{0}(x), \quad x \in M,
\end{aligned}
$$

where $W$ is a cylindrical Wiener process with nonlinear noise coefficient (operator) $B(u)$, the flux $f=f_{x}(\xi)$ is a vector field on $M$ depending (nonlinearly) on a real parameter $\xi$ and assumed to be geometry-compatible in the sense of Ben-Artzi and LeFloch [7], $\operatorname{div}_{h}$ is the divergence operator linked to $(M, h)$, the initial datum $u_{0}$ is a bounded (random) function, and $u=u(\omega, t, x)$ is the unknown that is sought up to a fixed final time $T>0$.

Our investigation of (1.1) utilizes firmly established tools for the analysis of (deterministic) conservation laws, specifically the kinetic formulation 54. As in Debussche and Vovelle [15], we make use of kinetic (and also generalized kinetic) solutions. Suppose for the moment that $W(t)$ is a one-dimensional Wiener process, and replace the operator $B(u)$ by a scalar function $g(x, u)$ that is (say) Lipschitz in both variables. In broad strokes, a process $u=u(\omega, t, x)$ is called a kinetic solution of (1.1) if the associated process

$$
\varrho(\omega, t, x, \xi)=\mathbb{I}_{u(\omega, t, x)>\xi}:= \begin{cases}1, & \text { if } \xi<u(\omega, t, x) \\ 0, & \text { if } \xi \geq u(\omega, t, x)\end{cases}
$$

satisfies (in the distributional sense) the kinetic equation

$$
\partial_{t} \varrho+\left(f_{x}^{\prime}(\xi), \nabla \varrho\right)_{h}+g(x, \xi) \partial_{\xi} \varrho \frac{d W}{d t}=\partial_{\xi}\left(\frac{(g(x, \xi))^{2}}{2} \partial_{\xi} \varrho\right)+\partial_{\xi} m,
$$

for some nonnegative (random) measure $m$, where $(\cdot, \cdot)_{h}$ is the inner product induced by the metric $h$. Note the property $\partial_{\xi} \varrho=-\delta(\xi-u)$ (and thus $\varrho(t, x, \cdot) \in$ $\left.B V_{\xi}\right)$. Roughly speaking, the difference between a kinetic solution $\varrho$ and a generalized kinetic solution $\rho$ is that this structural property is replaced by the requirement $\partial_{\xi} \rho=-\nu$ for some Young measure $\nu$ on $\mathbb{R}_{\xi}$ (that is parameterized over $\omega, t, x$ ). We refer to Section 3 for details.

Following an approach developed by Perthame 54 (instead of the "doubling of variables" method [15]), we establish a rigidity result implying that generalized kinetic solutions are in fact kinetic solutions, and that they are uniquely determined by their initial data ( $L^{1}$ contraction principle). To achieve this, we will employ a regularization procedure, commutator arguments àla DiPerna-Lions, and the Itô formula (for semimartingales) to show that a generalized kinetic solution $\rho$ and its square $\rho^{2}$ coincide (the "rigidity result"), provided $\left.\rho\right|_{t=0}=\mathbb{I}_{u_{0}>\xi}$. In our setting, added difficulties arise due to the stochastic forcing term and the nonlinear nature of the underlying domain $M$. In a nutshell, our strategy regarding the latter is the following: with the help of a partition of the unity, we will first localize the equation (1.1) and then "pull it back" to the Euclidean space, where the regularization procedure (convolution in $x, \xi$ ) will be carried out. This leads to several equations that are subsequently aggregated into a single (global) equation, living on the manifold $M$. Eventually this global equation is used to derive the rigidity result. We note that the solution to this equation is smooth in $x, \xi$ (but not $t$ ). The equation contains a commutator term (regularization error), arising as a result of the convective flux $f$ and the nonlinear nature of the domain $M$, which converges to zero thanks to a proper adaption of the DiPerna-Lions commutator lemma [21].

There are additional issues linked to the noise term in $(1.1)$ and its local time (quadratic covariation), including the handling of regularization errors tied to the $x$ and $\xi$ variables. For the moment, let us focus on the $\xi$ variable. For simplicity of 
presentation, we consider the Euclidean case and set $f \equiv 0$ (see Section 4 for the general case). To illustrate some of the difficulties, consider the kinetic equation

$$
\partial_{t} \varrho+a(\xi) \partial_{\xi} \varrho \frac{d W}{d t}=\partial_{\xi}\left(\frac{(b(\xi))^{2}}{2} \partial_{\xi} \varrho\right)+\partial_{\xi} m, \quad \varrho \text { of the form } 1.2 \text {, }
$$

where $a(\xi)$ and $b(\xi)$ are two, say, Lipschitz functions, noting that $b \equiv a$ corresponds to 1.3 . In order to compare $\varrho$ and $\varrho^{2}$, we need to determine the equation satisfied by $\varrho^{2}$. Let us attempt to do that for 1.4). Fix $S \in C^{2}$ (say, $S(f)=f^{2}$ ). A formal application of the Itô formula suggests the following equation for $S(\varrho)$ :

$$
\partial_{t} S(\varrho)+a(\xi) \partial_{\xi} S(\varrho) \frac{d W}{d t}=\partial_{\xi}\left(\frac{(b(\xi))^{2}}{2} \partial_{\xi} S(\varrho)\right)+S^{\prime}(\varrho) \partial_{\xi} m+\mathcal{Q}
$$

where $\mathcal{Q}$ contains the quadratic terms coming from the second order differential operator and the covariation of the martingale part of the equation $(1.4)$ :

$$
\mathcal{Q}=\left(a^{2}(\xi)-b^{2}(\xi)\right) \frac{S^{\prime \prime}(f)}{2}\left(\partial_{\xi} \varrho\right)^{2} .
$$

At first glance, it may seem that noise induces extra regularity in the $\xi$ variable, as a result of the second order differential operator in (1.4). This, however, is not the case. Only under the "super-parabolicity" condition $a^{2}(\cdot)<b^{2}(\cdot)$ do we have $Q \leq 0$, in which case $Q$ represents "dissipation" (from noise). The specific case $b \equiv a$ corresponds to the kinetic equation for the stochastic conservation law. The perfect cancellation (i.e., $Q=0$ ) in this case is the basic reason why the $L^{1}$ contraction principle (uniqueness) holds for these nonlinear SPDEs. Unfortunately the equation (1.5) is only suggestive (the calculations leading up to it are only formal). To make the calculations rigorous we regularize the linear equation (1.4) using a mollifier $\phi_{\delta}(x, \xi)$, thereby bringing in an additional type of regularization error $\mathcal{R}(\delta)$. If $a=b=g$ for some function $g(\cdot)$, then $\mathcal{R}(\delta)$ takes the form

$$
\left|\int \partial_{\xi} \varrho_{\delta}\left(\left(g^{2} \partial_{\xi} \varrho\right) \underset{(x, \xi)}{\star} \phi_{\delta}\right)-\left(\left(g \partial_{\xi} \varrho\right) \underset{(x, \xi)}{\star} \phi_{\delta}\right)^{2} d \xi d x\right|, \quad \varrho_{\delta}:=\varrho_{(x, \xi)}^{\star} \phi_{\delta} .
$$

Under a suitable regularity assumption on $g$, one can show that $\mathcal{R}(\delta) \rightarrow 0$ as $\delta \rightarrow 0$. The relevant assumption is dictated by the following derivable expression for $\mathcal{R}(\delta)$ :

$$
\begin{aligned}
\mathcal{R}(\delta)=\frac{1}{2} \int \mid & g(\zeta)-\left.g(\bar{\zeta})\right|^{2} \partial_{\xi} \varrho(t, y, \zeta) \partial_{\xi} \varrho(t, \bar{y}, \bar{\zeta}) \\
& \times \phi_{\delta}(x-y, \xi-\zeta) \phi_{\delta}(x-\bar{y}, \xi-\bar{\zeta}) d \zeta d \bar{\zeta} d y d \bar{y} d x d \xi
\end{aligned}
$$

For a standard mollifier $\phi_{\delta}$, it turns out that this expression tends to zero as $\delta \rightarrow 0$ if $g$ is Lipschitz, or more generally if $\left|g\left(\xi_{1}\right)-g\left(\xi_{2}\right)\right|^{2} \leq C\left|\xi_{1}-\xi_{2}\right| \delta\left(\left|\xi_{1}-\xi_{2}\right|\right)$ for some continuous function $\delta$ on $\mathbb{R}_{+}$with $\delta(0)=0$, which is consistent with [15]. Up to this point we have tried to extract some of the main ideas behind the uniqueness proof (in a simplified situation). Unfortunately, the complete proof in the general case is painfully long and technical. We refer to Section 4 for details.

As part of showing existence of kinetic solutions, we will establish the wellposedness of variational solutions [42] for a stochastic parabolic problem, obtained by adding to (1.1) a small diffusion term $\varepsilon \Delta_{h}(\varepsilon>0)$ involving the LaplaceBeltrami operator $\Delta_{h}$ on $(M, h)$ (cf. Section 5 for details). Making use of a priori $\left(L^{p}\right)$ estimates, we prove that there exists a kinetic solution to (1.1) by arguing that the kinetic function linked to the variational solution (of the stochastic parabolic equation) converges weakly as $\varepsilon \rightarrow 0$ to a generalized kinetic solution of (1.1) (cf. Section 7 for details). A crucial ingredient in the overall existence proof is a generalized Itô formula for weak solutions to a wide class of SPDEs on Riemannian manifolds. Indeed, since variational solutions of the stochastic parabolic equation 
are merely $H^{1}$ regular in the $x$ variable, our general setting forces us to derive this Itô formula. This is the topic of Section 6 .

\section{BACKGROUND AND HYPOTHESES}

We now provide the precise assumptions on each of the terms appearing in the stochastic conservation law (1.1). Basic background material on hyperbolic conservation laws can be found in the books [11, 54].

2.1. Geometric framework. The underlying space is an $n$-dimensional $(n \geq 1)$ smooth manifold $M$, which we assume to be compact, connected, oriented and with no boundary. Moreover, $M$ is endowed with a smooth Riemannian metric $h$. By this, we mean that $h$ is a positive-definite, 2-covariant tensor field, which thus determines for every $x \in M$ an inner product $h_{x}$ on $T_{x} M$ (the tangent space at $x$ ). For any two vectors $V_{1}, V_{2} \in T_{x} M$, we will henceforth write $h_{x}\left(V_{1}, V_{2}\right)=:\left(V_{1}, V_{2}\right)_{h_{x}}$ or even $\left(V_{1}, V_{2}\right)_{h}$ if the context is clear. We set $|V|_{h}:=(V, V)_{h}^{1 / 2}$. Recall that in local coordinates $x=\left(x^{i}\right)$, the derivations $\partial_{i}:=\frac{\partial}{\partial x^{i}}$ form a basis for $T_{x} M$, while the differential forms $d x^{i}$ determine a basis for the cotangent space $T_{x}^{*} M$. Therefore, in local coordinates, $h$ reads

$$
h=h_{i j} d x^{i} d x^{j}, \quad h_{i j}=\left(\partial_{i}, \partial_{j}\right)_{h},
$$

Here and elsewhere we employ the Einstein summation convention over repeated indices. We will denote by $\left(h^{i j}\right)$ the inverse of the matrix $\left(h_{i j}\right)$.

We denote by $d V_{h}$ the Riemannian density associated to $h$, which in local coordinates reads

$$
d V_{h}=|h|^{1 / 2} d x^{1} \cdots d x^{n},
$$

where $|h|$ is the determinant of $h$. We recall that integration with respect to $d V_{h}$ is done in the following way: if $u \in C^{0}(M)$ has support contained in the domain of a single chart $\Phi: U \subset M \rightarrow \Phi(U) \subset \mathbb{R}^{n}$, then

$$
\int_{M} u(x) d V_{h}(x)=\int_{\Phi(U)}\left(|h|^{1 / 2} u\right) \circ \Phi^{-1} d x^{1} \cdots d x^{n},
$$

where $\left(x^{i}\right)$ are the coordinates associated to $\Phi$. If supp $u$ is not contained in a single chart domain, then the integral is defined as

$$
\int_{M} u(x) d V_{h}(x)=\sum_{i \in \mathcal{I}} \int_{M} \alpha_{i} u d V_{h}(x),
$$

where $\left(\alpha_{i}\right)_{i \in \mathcal{I}}$ is a partition of unity subordinate to some atlas $\mathcal{A}$. Throughout the paper, we will assume for convenience that

$$
\operatorname{Vol}(M, h):=\int_{M} d V_{h}=1
$$

Always in local coordinates, the gradient of a function $u: M \rightarrow \mathbb{R}$ is the vector field given by the following expression

$$
\operatorname{grad}_{h} u:=h^{i j} \partial_{i} u \partial_{j} .
$$

The symbol $\nabla$ will indicate the Levi-Civita connection of $h$, namely the unique linear connection on $M$ that is compatible with $h$ and is symmetric. In particular, the covariant derivative of a vector field $X=X^{\alpha} \partial_{\alpha}$ is the $(1,1)$-tensor field which in local coordinates takes the following form

$$
(\nabla X)_{j}^{\alpha}=X_{; j}^{\alpha}:=\partial_{j} X^{\alpha}+\Gamma_{k j}^{\alpha} X^{k},
$$

where $\Gamma_{i j}^{k}$ are the Christoffel symbols associated to $\nabla$ :

$$
\Gamma_{i j}^{k}=\frac{1}{2} h^{k l}\left(\partial_{i} h_{j l}+\partial_{j} h_{i l}-\partial_{l} h_{i j}\right) .
$$


The divergence of a vector field $X$ is the function defined by

$$
\operatorname{div}_{h} X=\partial_{j} X^{j}+\Gamma_{k j}^{j} X^{k}
$$

We recall that for a function $u \in C^{1}(M)$ and a smooth vector field $X$, the following integration by parts formula holds:

$$
\int_{M}\left(\operatorname{grad}_{h} u, X\right)_{h} d V_{h}=-\int_{M} u \operatorname{div}_{h} X d V_{h} .
$$

We assume that $f=f_{x}(\xi)$ is a vector field on $M$ depending on the real parameter $\xi$. More precisely, $f: M \times \mathbb{R} \rightarrow T M$, where $T M$ is the tangent bundle of $M$, and $f$ is smooth in both $x$ and $\xi$. We will call $f$ the flux on $M$. Following [7, we assume that $f$ is geometry-compatible, in the sense that

$$
\operatorname{div}_{h} f_{x}(\xi)=0, \quad \xi \in \mathbb{R}, \quad x \in M .
$$

Moreover, we impose the following polynomial growth conditions on $f$ and the derivative $f^{\prime}:=\partial_{\xi} f:$

$$
\begin{cases}\left|f_{x}(\xi)\right|_{h} \leq C_{0}\left(1+|\xi|^{r}\right), & \xi \in \mathbb{R}, x \in M \\ \left|f_{x}^{\prime}(\xi)\right|_{h} \leq C_{0}\left(1+|\xi|^{r-1}\right), & \xi \in \mathbb{R}, x \in M,\end{cases}
$$

for some constants $C_{0}>0, r \geq 1$.

We denote by $L^{p}(M, h), p \geq 1$ the usual Lebesgue spaces on $(M, h)$. The Sobolev spaces $H^{k}(M, h), k \geq 1$, are defined as the completion of $C^{\infty}(M)$ with respect to the norm

$$
\|u\|_{H^{k}(M, h)}=\left(\sum_{j=0}^{k} \int_{M}\left|\nabla^{j} u\right|_{h}^{2} d V_{h}\right)^{\frac{1}{2}},
$$

where

$$
\left|\nabla^{j} u\right|_{h}^{2}=h^{a_{1} b_{1}} \ldots h^{a_{j} b_{j}}\left(\nabla^{j} u\right)_{a_{1} \cdots a_{j}}\left(\nabla^{j} u\right)_{b_{1} \cdots b_{j}}
$$

(in a local chart) and $\nabla^{j}$ designates the $j^{\text {th }}$ covariant derivative of $u$. Note that the spaces $H^{k}(M, h)$ are Hilbert spaces. For further details, we refer to [3] and [34.

Note that, for $x \in M$ and $V \in T_{x} M$, it holds $(\nabla u)(V)=\left(\operatorname{grad}_{h} u, V\right)_{h}$. For this reason, we will in this paper slightly abuse the notation by always writing $(\nabla u, V)_{h}$ instead of $\left(\operatorname{grad}_{h} u, V\right)_{h}$; that is, we identify $\operatorname{grad}_{h} u$ and $\nabla u$.

2.2. Stochastic framework. For background material on stochastic analysis and SPDEs, we refer to the books [13, 55]. For a topological space $(X, \tau)$, the symbol $\mathcal{B}(X)$ will indicate its Borel $\sigma$-algebra. Given two measurable spaces $\left(X_{i}, \mathcal{M}_{i}\right), i=$ 1,2 , and a map $f: X_{1} \rightarrow X_{2}$, the expression " $f$ is $\mathcal{M}_{1} / \mathcal{M}_{2}$ measurable" (or simply " $f$ is $\mathcal{M}_{1} / \mathcal{M}_{2}$ ") means that $f^{-1}(B) \in \mathcal{M}_{1}$ for all $B \in \mathcal{M}_{2}$.

Regarding the stochastic term, we are given a complete probability space $(\Omega, \mathcal{F}, \mathbb{P})$, along with a complete right-continuous filtration $\left(\mathcal{F}_{t}\right)_{t \geq 0}$. We denote by $\mathcal{P}$ the predictable $\sigma$-algebra on $\Omega_{T}:=\Omega \times[0, T]$ (associated to $\left(\mathcal{F}_{t}\right)_{t \geq 0}$ ). By this, we mean

$$
\begin{aligned}
\mathcal{P} & =\sigma\left(\left\{(s, t] \times F_{s}: 0 \leq s<t \leq T, F_{s} \in \mathcal{F}_{s}\right\} \cup\left\{\{0\} \times F_{0}: F_{0} \in \mathcal{F}_{0}\right\}\right) \\
& =\sigma\left(Y: \Omega_{T} \rightarrow \mathbb{R}: Y \text { is left-continuous and adapted to } \mathcal{F}_{t}, t \in[0, T]\right) .
\end{aligned}
$$

Given an arbitrary separable Hilbert space $\tilde{H}$, a map $Y: \Omega_{T} \rightarrow \tilde{H}$ that is $\mathcal{P} / \mathcal{B}(\tilde{H})$ measurable will be called $\tilde{H}$-predictable. If $(X, \mathcal{M})$ is a measure space, a map $Y: \Omega_{T} \times X \rightarrow \tilde{H}$ will be called progressively measurable (with respect to $\left(\mathcal{F}_{t}\right)_{t \geq 0}$ ) if for all $t \in[0, T]$ the map $\left.Y\right|_{\Omega \times[0, t] \times X}$ is $\mathcal{F}_{t} \otimes \mathcal{B}([0, t]) \otimes \mathcal{M} / \mathcal{B}(\tilde{H})$ measurable.

Whenever we write that a statement holds true "for a.e. $(\omega, t)$ ", we will be referring to the product measure between $\mathbb{P}$ and the Lebesgue measure on $[0, T]$. 
The initial datum $u_{0}$ is in general a random variable, namely $u_{0}$ is $\mathcal{F}_{0}$-measurable and in $L^{p}\left(\Omega ; L^{p}(M, h)\right)$ for some $p \in[1, \infty)$. The driving process $W$ is a cylindrical Wiener process, i.e., $W(t)=\sum_{k \geq 1} \beta_{k}(t) e_{k}$, where

(1) $\left(e_{k}\right)_{k \geq 1}$ is an orthonormal basis for a separable Hilbert space $\mathfrak{U}$;

(2) $\left(\beta_{k}(t)\right)_{k \geq 1}$ are mutually independent real-valued standard Wiener processes relative to $\left(\mathcal{F}_{t}\right)_{t \geq 0}$

(3) the sum converges in $\mathcal{M}_{T}^{2}\left(\mathfrak{U}_{0}\right)$, the space of $\mathfrak{U}_{0}$-valued continuous, square integrable martingales, where $\mathfrak{U}_{0}$ is the auxiliary Hilbert space defined as

$$
\mathfrak{U}_{0}:=\left\{v=\sum_{k \geq 1} a_{k} e_{k}: \sum_{k \geq 1} \frac{a_{k}^{2}}{k^{2}}<\infty\right\},
$$

endowed with the norm

$$
\|v\|_{\mathfrak{U}_{0}}^{2}=\sum_{k \geq 1} \frac{a_{k}^{2}}{k^{2}}
$$

such that the embedding $\mathfrak{U} \hookrightarrow \mathfrak{U}_{0}$ is Hilbert-Schmidt (cf. 55] for details).

In our setting, we can assume without loss of generality that the $\sigma$-algebra $\mathcal{F}$ is countably generated and $\left(\mathcal{F}_{t}\right)_{t \in[0, T]}$ is the filtration generated by $u_{0}$ and $W$.

For each $z \in L^{2}(M, h)$, we consider a mapping $B(z): \mathfrak{U} \rightarrow L^{2}(M, h)$ defined by $B(z) e_{k}:=g_{k}(\cdot, z(\cdot)), k \in \mathbb{N}$, with $g_{k} \in C^{0}(M \times \mathbb{R})$. We assume the following conditions on $\left\{g_{k}\right\}_{k \in \mathbb{N}}$ : there exist positive constants $D_{1}, D_{2}$ such that

$$
\begin{aligned}
& G^{2}(x, \xi):=\sum_{k \geq 1}\left|g_{k}(x, \xi)\right|^{2} \leq D_{1}\left(1+|\xi|^{2}\right), x \in M, \xi \in \mathbb{R} \\
& \sum_{k \geq 1}\left|g_{k}(x, \xi)-g_{k}(y, \zeta)\right|^{2} \leq D_{2}\left(d_{h}^{2}(x, y)+|\xi-\zeta|^{2}\right), \quad x, y \in M, \xi, \zeta \in \mathbb{R}
\end{aligned}
$$

where $d_{h}$ is the distance function on $(M, h)$. From $(2.3)$, it easily follows that

$$
B: L^{2}(M, h) \rightarrow L_{2}\left(\mathfrak{U} ; L^{2}(M, h)\right),
$$

where $L_{2}\left(\mathfrak{U} ; L^{2}(M, h)\right)$ denotes the (separable Hilbert) space of Hilbert-Schmidt operators from $\mathfrak{U}$ to $L^{2}(M, h)$. We also observe that, in view of (2.4), $B$ is Lipschitz on $L^{2}(M, h)$, because it holds, for any $z_{1}, z_{2} \in L^{2}(M, h)$,

$$
\begin{aligned}
& \left\|B\left(z_{1}\right)-B\left(z_{2}\right)\right\|_{L_{2}\left(\mathfrak{U} ; L^{2}(M, h)\right)}^{2}=\sum_{k \geq 1}\left\|B\left(z_{1}\right) e_{k}-B\left(z_{2}\right) e_{k}\right\|_{L^{2}(M, h)}^{2} \\
& =\int_{M} \sum_{k \geq 1}\left|g_{k}\left(x, z_{1}(x)\right)-g_{k}\left(x, z_{2}(x)\right)\right|^{2} d V_{h}(x) \\
& \quad \leq \int_{M} D_{2}\left|z_{1}(x)-z_{2}(x)\right|^{2} d V_{h}(x)=D_{2}\left\|z_{1}-z_{2}\right\|_{L^{2}(M, h)}^{2} .
\end{aligned}
$$

For later use, we note the following simple result:

Lemma 2.1. Assume that 2.3 and 2.4 hold. Then

$$
\begin{aligned}
& \left|G^{2}\left(x_{1}, \xi_{1}\right)-G^{2}\left(x_{2}, \xi_{2}\right)\right| \\
& \quad \leq \sqrt{D_{1} D_{2}}\left\{\sqrt{1+\xi_{1}^{2}}+\sqrt{1+\xi_{2}^{2}}\right\} \sqrt{d_{h}^{2}\left(x_{1}, x_{2}\right)+\left|\xi_{1}-\xi_{2}\right|^{2}},
\end{aligned}
$$

for all $x_{1}, x_{2} \in M$ and $\xi_{1}, \xi_{2} \in \mathbb{R}$. 
Proof. A direct computation gives

$$
\begin{aligned}
& \left|G^{2}\left(x_{1}, \xi_{1}\right)-G^{2}\left(x_{2}, \xi_{2}\right)\right| \leq \sum_{k \geq 1}\left|g_{k}^{2}\left(x_{1}, \xi_{1}\right)-g_{k}^{2}\left(x_{2}, \xi_{2}\right)\right| \\
& \quad \leq\left(\sum_{k \geq 1}\left|g_{k}\left(x_{1}, \xi_{1}\right)+g_{k}\left(x_{2}, \xi_{2}\right)\right|^{2}\right)^{1 / 2}\left(\sum_{k \geq 1}\left|g_{k}\left(x_{1}, \xi_{1}\right)-g_{k}\left(x_{2}, \xi_{2}\right)\right|^{2}\right)^{1 / 2} \\
& \quad \leq \sqrt{D_{1} D_{2}}\left\{\sqrt{1+\xi_{1}^{2}}+\sqrt{1+\xi_{2}^{2}}\right\} \sqrt{d_{h}^{2}\left(x_{1}, x_{2}\right)+\left|\xi_{1}-\xi_{2}\right|^{2}} .
\end{aligned}
$$

In (1.1), " $B(u) d W "$ is understood as an Itô stochastic integral. We refer to [13, 55] for a detailed construction of the stochastic integral

$$
N_{t}:=\int_{0}^{t} H d W=\sum_{k \geq 1} \int_{0}^{t} H_{k} d \beta_{k}, \quad H_{k}:=H e_{k},
$$

for any predictable $L^{2}(M, h)$-valued process

$$
H \in L^{2}\left(\Omega, \mathcal{F} ; L^{2}\left(0, T ; L_{2}\left(\mathfrak{U} ; L^{2}(M, h)\right)\right)\right) .
$$

We will frequently make use of the Burkholder-Davis-Gundy inequality [55, App. D], which applied to $N_{t}$ reads

$$
\mathbb{E}\left[\sup _{t \in[0, T]}\left\|\sum_{k \geq 1} \int_{0}^{t} H_{k} d \beta_{k}\right\|_{L^{2}(M, h)}^{p}\right] \leq C \mathbb{E}\left[\left(\int_{0}^{T} \sum_{k \geq 1}\left\|H_{k}\right\|_{L^{2}(M, h)}^{2} d t\right)^{\frac{p}{2}}\right]
$$

where $C$ is a constant depending on $p \geq 1$.

Remark 2.1. Condition 2.4 is used for convenience and simplicity of presentation. It can be replaced by the following more general condition (conforming to [15]):

$$
\sum_{k \geq 1}\left|g_{k}(x, \xi)-g_{k}(y, \zeta)\right|^{2} \leq D_{2}\left(d_{h}^{2}(x, y)+|\xi-\zeta| \delta(|\xi-\zeta|)\right),
$$

for $x, y \in M$ and $\xi, \zeta \in \mathbb{R}$, where $\delta:[0, \infty) \rightarrow[0, \infty)$ is a continuous non-decreasing function such that $\delta(0)=0$. The relevant proofs remain the same modulo some notational changes.

\section{Kinetic SOlutions And MAin RESUlt}

Following Debussche and Vovelle [15, we introduce the concepts of kinetic and generalized kinetic solutions for stochastic conservation laws defined on a manifold. We start with the notion of kinetic measure.

Definition 3.1 (kinetic measure). We say that a map $m$ from $\Omega$ to the set of non-negative finite measures over $[0, T] \times M \times \mathbb{R}$ is a kinetic measure if

(1) $m$ is measurable, that is, for each $\phi \in C_{b}^{0}([0, T] \times M \times \mathbb{R}), m(\phi): \Omega \rightarrow \mathbb{R}$ is measurable, where $m(\phi)$ denotes the action of $m$ on $\phi$;

(2) $m$ is integrable, that is,

$$
\mathbb{E} m([0, T] \times M \times \mathbb{R})<\infty ;
$$

(3) $m$ vanishes for large $\xi$, that is, if $B_{R}^{c}=\{\xi \in \mathbb{R}:|\xi| \geq R\}$, then

$$
\lim _{R \rightarrow \infty} \mathbb{E} m\left([0, T] \times M \times B_{R}^{c}\right)=0 ;
$$


(4) for all $\phi \in C_{b}^{0}(M \times \mathbb{R})$, the process

$$
t \mapsto \int_{[0, t] \times M \times \mathbb{R}} \phi(x, \xi) m(d s, d x, d \xi) \in L^{2}(\Omega \times[0, T])
$$

admits a predictable representative.

Definition 3.2 (kinetic solutions). With $u_{0} \in L^{\infty}\left(\Omega, \mathcal{F}_{0} ; L^{\infty}(M, h)\right)$, set $\rho_{0}:=$ $\mathbb{I}_{u_{0}>\xi}$. A measurable function $u: \Omega \times[0, T] \times M \rightarrow \mathbb{R}$ is said to be a kinetic solution of (1.1) with initial data $u_{0}$ if $(u(t))_{t \in[0, T]}$ is predictable; $\forall p \in[1, \infty)$, there exists a positive constant $C_{p}$ such that

$$
\mathbb{E}\left(\operatorname{ess}_{t \in[0, T]}\|u(t)\|_{L^{p}(M, h)}^{p}\right) \leq C_{p}
$$

and there exists a kinetic measure $m$ such that $\mathbb{P}$-a.s. the function $\rho:=\mathbb{I}_{u>\xi}$ satisfies

$$
\begin{aligned}
\int_{0}^{T} & \int_{M} \int_{\mathbb{R}} \rho \partial_{t} \psi d \xi d V_{h}(x) d t+\int_{M} \int_{\mathbb{R}} \rho_{0} \psi(0, x, \xi) d \xi d V_{h}(x) \\
& +\int_{0}^{T} \int_{M} \int_{\mathbb{R}} \rho\left(f_{x}^{\prime}(\xi), \nabla \psi\right)_{h} d \xi d V_{h}(x) d t=m\left(\partial_{\xi} \psi\right) \\
& -\sum_{k \geq 1} \int_{0}^{T} \int_{M} g_{k}(x, u(t, x)) \psi(t, x, u(t, x)) d V_{h}(x) d \beta_{k}(t) \\
& -\frac{1}{2} \int_{0}^{T} \int_{M} \partial_{\xi} \psi(t, x, u(t, x)) G^{2}(x, u(t, x)) d V_{h}(x) d t
\end{aligned}
$$

for all $\psi \in C_{c}^{1}([0, T) \times M \times \mathbb{R})$.

Let $(X, \mu)$ be a finite measure space, and denote by $\operatorname{Prob}(\mathbb{R})$ the set of probability measures on $\mathbb{R}$. A map $\nu: X \rightarrow \operatorname{Prob}(\mathbb{R})$ is a Young measure on $X$ if, for all $\phi \in C_{b}(\mathbb{R})$, the map $z \mapsto \nu_{z}(\phi)$ from $X$ to $\mathbb{R}$ is measurable. We say that a Young measure $\nu$ vanishes at infinity if, for every $p \in[1, \infty)$,

$$
\int_{X} \int_{\mathbb{R}}|\xi|^{p} \nu_{z}(d \xi) \mu(d z)<\infty .
$$

Let $(X, \mu)$ be a finite measure space. A measurable function $\rho: X \times \mathbb{R} \rightarrow[0,1]$ is said to be a generalized kinetic function if there exists a Young measure $\nu$ on $X$ vanishing at infinity such that, for $\mu$-a.e. $z \in X$ and for all $\xi \in \mathbb{R}, \rho(z, \xi)=\nu_{z}(\xi, \infty)$.

We say that $\rho$ is a kinetic function if there exists a measurable function $u: X \rightarrow \mathbb{R}$ such that $\rho(z, \xi)=\mathbb{I}_{u(z)>\xi}$ a.e., or, equivalently, $\nu_{z}=\delta_{u(z)}$ for $\mu$-a.e. $z \in X$.

A generalized kinetic function $\rho$ satisfies $\partial_{\xi} \rho=-\nu$. If $\rho$ is a kinetic function, then $\partial_{\xi} \rho=-\delta_{u}$. Let $\rho$ be a generalized kinetic function. Note that the function $\chi_{\rho}(z, \xi):=\rho(z, \xi)-\mathbb{I}_{0>\xi}$ is, contrary to $\rho$, integrable on $\mathbb{R}_{\xi}$.

Definition 3.3 (generalized kinetic solution). Fix a generalized kinetic function $\rho_{0}: \Omega \times M \times \mathbb{R} \rightarrow[0,1]$. We call $\rho: \Omega \times[0, T] \times M \times \mathbb{R} \rightarrow[0,1]$ a generalized kinetic solution of 1.1 with initial data $\rho_{0}$ if $\chi_{\rho}=\rho-\mathbb{I}_{0>\xi}$ is $\mathcal{P} / \mathcal{B}\left(L^{2}(M \times \mathbb{R})\right)$ measurable and for all $p \in[1, \infty)$ there exists $C_{p}>0$ such that

$$
\mathbb{E}\left(\operatorname{ess}_{t \in[0, T]} \int_{M} \int_{\mathbb{R}}|\xi|^{p} \nu_{\omega, t, x}(d \xi) d V_{h}(x)\right) \leq C_{p}
$$


where $\nu=-\partial_{\xi} \rho$ is a Young measure, and if there exists a kinetic measure $m$ such that $\mathbb{P}$-a.s. $\left(f^{\prime}=\partial_{\xi} f\right)$

$$
\begin{gathered}
\int_{0}^{T} \int_{M} \int_{\mathbb{R}} \rho \partial_{t} \psi d \xi d V_{h}(x) d t+\int_{M} \int_{\mathbb{R}} \rho_{0} \psi(0, x, \xi) d \xi d V_{h}(x) \\
\quad+\int_{0}^{T} \int_{M} \int_{\mathbb{R}} \rho\left(f_{x}^{\prime}(\xi), \nabla \psi\right)_{h} d \xi d V_{h}(x) d t=m\left(\partial_{\xi} \psi\right) \\
\quad-\sum_{k \geq 1} \int_{0}^{T} \int_{M} \int_{\mathbb{R}} g_{k}(x, \xi) \psi \nu_{\omega, t, x}(d \xi) d V_{h}(x) d \beta_{k}(t) \\
-\frac{1}{2} \int_{0}^{T} \int_{M} \int_{\mathbb{R}} \partial_{\xi} \psi G^{2}(x, \xi) \nu_{\omega, t, x}(d \xi) d V_{h}(x) d t
\end{gathered}
$$

for all $\psi \in C_{c}^{1}([0, T) \times M \times \mathbb{R})$.

We note the following result, which is identical to [15, Proposition 10] (see also [11, Lemma 1.3.3]). It tells us that a generalized kinetic solution possesses (weak) left and right limits at every instant of time.

Lemma 3.1. Let $\rho$ be a generalized kinetic solution to 1.1 with initial data $\rho_{0}$. For any $t_{*} \in[0, T]$, there exist generalized kinetic functions $\rho^{*, \pm}$ on $\Omega \times M \times \mathbb{R}$ such that $\mathbb{P}$-a.s.,

$$
\begin{gathered}
\iint_{M \times \mathbb{R}} \rho\left(t_{*}-h\right) \psi d \xi d V_{h}(x) \stackrel{h \downarrow 0}{\rightarrow} \iint_{M \times \mathbb{R}} \rho^{*,-} \psi d \xi d V_{h}(x), \\
\iint_{M \times \mathbb{R}} \rho\left(t_{*}+h\right) \psi d \xi d V_{h}(x) \stackrel{h \downarrow 0}{\rightarrow} \iint_{M \times \mathbb{R}} \rho^{*,+} \psi d \xi d V_{h}(x),
\end{gathered}
$$

for all $\psi \in C_{c}^{1}(M \times \mathbb{R})$. Moreover, $\mathbb{P}$-a.s.,

$$
\iint_{M \times \mathbb{R}}\left(\rho^{*,+}-\rho^{*,-}\right) \psi d \xi d V_{h}(x)=-\int_{[0, T] \times M \times \mathbb{R}} \partial_{\xi} \psi(x, \xi) \mathbb{I}_{\left\{t_{*}\right\}}(t) m(d t, d x, d \xi) .
$$

In particular, $\mathbb{P}$-a.s., the set $\left\{t_{*} \in[0, T]: \rho^{*,+} \neq \rho^{*,-}\right\}$ is at most countable.

For a proof of this result see the above-mentioned reference. For a generalized kinetic solution $\rho$, we henceforth define the accompanying functions $\rho^{ \pm}$by setting $\rho^{ \pm}\left(t_{*}\right)=\rho^{*, \pm}\left(t_{*}\right)$ for $t_{*} \in[0, T]$. Clearly, $\rho^{+}(t)=\rho^{-}(t)=\rho(t)$ for a.e. $t \in[0, T]$.

As in 15, page 14], we can replace $(3.2)$ by a weak formulation that is pointwise in time: $\forall t \in[0, T]$ and $\forall \psi \in C_{c}^{1}(M \times \mathbb{R})$,

$$
\begin{aligned}
-\int_{M} \int_{\mathbb{R}} \rho^{+}(t) \psi d \xi d V_{h}(x)+\int_{M} \int_{\mathbb{R}} \rho_{0} \psi d \xi d V_{h}(x) \\
+\int_{0}^{t} \int_{M} \int_{\mathbb{R}} \rho^{+}(s)\left(f_{x}^{\prime}(\xi), \nabla \psi\right)_{h} d \xi d V_{h}(x) d s \\
=-\sum_{k \geq 1} \int_{0}^{t} \int_{M} \int_{\mathbb{R}} g_{k}(x, \xi) \psi \nu_{\omega, s, x}(d \xi) d V_{h}(x) d \beta_{k}(s) \\
-\frac{1}{2} \int_{0}^{t} \int_{M} \int_{\mathbb{R}} \partial_{\xi} \psi G^{2}(x, \xi) \nu_{\omega, s, x}(d \xi) d V_{h}(x) d s \\
+\int_{[0, t] \times M \times \mathbb{R}} \partial_{\xi} \psi m(d s, d x, d \xi), \quad \mathbb{P} \text {-almost surely. }
\end{aligned}
$$

This "pointwise in time" formulation will be utilized in the uniqueness proof.

The next theorem contains the main result of the paper, namely the existence, uniqueness, and stability of kinetic solutions. Moreover, the trajectories (in $L^{p}$ ) of kinetic solution are continuous, $\mathbb{P}$-almost surely. The proof of the theorem is scattered across Section 4 (uniqueness) and Section 7 (existence). 
Theorem 3.2 (well-posedness). Suppose 2.2, 2.3), 2.4 hold. There exists a unique kinetic solution $u$ of $\sqrt{1.1}$ with initial datum $u_{0} \in L^{\infty}\left(\Omega, \mathcal{F}_{0} ; L^{\infty}(M, h)\right)$. If $u_{1}, u_{2}$ are kinetic solutions of (1.1) with initial data $u_{1,0}, u_{2,0}$, respectively, then the following $L^{1}$ contraction property holds:

$$
\mathbb{E} \int_{M}\left|\left(u_{1}-u_{2}\right)(t, x)\right| d V_{h}(x) \leq \mathbb{E} \int_{M}\left|\left(u_{1,0}-u_{2,0}\right)(x)\right| d V_{h}(x),
$$

for $t \in[0, T]$. Besides, $u$ has a representative in $L^{p}\left(\Omega ; L^{\infty}\left(0, T ; L^{p}(M, h)\right)\right)$ with continuous trajectories in $L^{p}(M, h), \mathbb{P}$-a.s., for any $p \in[1, \infty)$.

\section{Rigidity AND UNIQUENESS RESUlts}

The aim of this section is to show that generalized kinetic solutions are in fact kinetic solutions and that they are unique. To achieve this, we will employ a regularization procedure, which will enable us to compare $\left(\rho^{ \pm}\right)^{2}$ and $\rho^{ \pm}$, following [54] (see also [12]). Our strategy is the following: with the help of a smooth partition of unity subordinate to a finite atlas $\mathcal{A}=\left\{\kappa: X_{\kappa} \rightarrow \tilde{X}_{\kappa}\right\}_{\kappa}$, we localize the equation (3.3) on $X_{\kappa} \subset M$, thereby obtaining $\sharp(\mathcal{A})$ equations, indexed by $\kappa$, that are "pulled back" to $\tilde{X}_{\kappa} \subset \mathbb{R}^{n}$ and regularized with a mollifier on $\mathbb{R}^{n} \times \mathbb{R}$. Subsequently, we aggregate the regularized equations to arrive at a single SPDE, parameterized by $(x, \xi) \in M \times \mathbb{R}$. We renormalize this equation using Itô's formula. This enables us to analyze the difference between the regularized versions of $\rho^{+}$and $\left(\rho^{+}\right)^{2}$, eventually concluding the rigidity result.

The key result of this section is

Proposition 4.1 (rigidity result). Suppose (2.2), 2.3), and (2.4) hold. Let $\rho$ be a generalized kinetic solution to (1.1) with initial data $\rho_{0}$. Then, for all $t \in[0, T]$,

$$
\mathbb{E} \int_{M \times \mathbb{R}}\left(\rho^{+}-\left(\rho^{+}\right)^{2}\right)(t) d \xi d V_{h}(x) \leq \mathbb{E} \int_{M \times \mathbb{R}}\left(\rho_{0}-\rho_{0}^{2}\right) d \xi d V_{h}(x),
$$

where the temporal right limit $\rho^{+}$of $\rho$ is defined in Lemma 3.1 .

Note that if $\rho_{0}$ is a kinetic function, i.e., $\rho_{0}=\mathbb{I}_{u_{0}>\xi}$ for some bounded random function $u_{0}$, then $\rho^{+}-\left(\rho^{+}\right)^{2}=0$ (rigidity) and accordingly $\rho^{+}$takes values in $\{0,1\}$. Consequently, $\rho^{+}$is a kinetic function, i.e., there exists a measurable function $u$ such that $\rho=\mathbb{I}_{u>\xi}$ (and Theorem 3.2 will follow from this).

The proof of Proposition 4.1 will be laid out in several subsections.

4.1. Localized equations. To prove Proposition 4.1. we need some preparational material. We will work with a finite atlas $\mathcal{A}=\left\{\kappa: X_{\kappa} \rightarrow \tilde{X}_{\kappa}\right\}_{\kappa}$, where $X_{\kappa} \subset M$ is an open subset of $M$ and $\tilde{X}_{\kappa} \subset \mathbb{R}^{n}$ is an open subset of $\mathbb{R}^{n}$. The typical point of $\mathbb{R}^{n}$ will be denoted by $z$. We take a smooth partition of the unity $\left\{\alpha_{\kappa}\right\}_{\kappa \in \mathcal{A}}$ subordinate to $\mathcal{A}$, such that

(1) $\alpha_{\kappa} \geq 0, \sum_{\kappa \in \mathcal{A}} \alpha_{\kappa}=1$,

(2) $\alpha_{\kappa} \in C^{\infty}(M)$, and

(3) $\operatorname{supp} \alpha_{\kappa} \subset X_{\kappa}$ (and compact).

Let $\rho$ be a generalized kinetic solution with initial data $\rho_{0}$ (not necessarily of the form $I_{u_{0}>\xi}$ ). As $\alpha_{\kappa} \in C^{\infty}(M)$, it follows from (3.3) that the function $\alpha_{\kappa} \rho^{+}(t)$ 
solves for all $\psi \in C_{c}^{1}(M \times \mathbb{R})$ and $t \in[0, T]$,

$$
\begin{aligned}
-\int_{M} \int_{\mathbb{R}} & \alpha_{\kappa}(x) \rho^{+}(t) \psi d \xi d V_{h}(x)+\int_{M} \int_{\mathbb{R}} \alpha_{\kappa}(x) \rho_{0} \psi d \xi d V_{h}(x) \\
+ & \int_{0}^{t} \int_{M} \int_{\mathbb{R}} \alpha_{\kappa}(x) \rho^{+}(s)\left(f_{x}^{\prime}(\xi), \nabla \psi\right)_{h} d \xi d V_{h}(x) d s \\
& +\int_{0}^{t} \int_{M} \int_{\mathbb{R}} \rho^{+}(s) \psi\left(f_{x}^{\prime}(\xi), \nabla \alpha_{\kappa}\right)_{h} d \xi d V_{h}(x) d s \\
=- & \sum_{k \geq 1} \int_{0}^{t} \int_{M} \int_{\mathbb{R}} g_{k}(x, \xi) \psi \alpha_{\kappa}(x) \nu_{\omega, s, x}(d \xi) d V_{h}(x) d \beta_{k}(s) \\
& -\frac{1}{2} \int_{0}^{t} \int_{M} \int_{\mathbb{R}} \partial_{\xi} \psi G^{2}(x, \xi) \alpha_{\kappa}(x) \nu_{\omega, s, x}(d \xi) d V_{h}(x) d s \\
& +\int_{[0, t] \times M \times \mathbb{R}} \partial_{\xi} \psi \alpha_{\kappa}(x) m(d s, d x, d \xi), \quad \mathbb{P} \text {-almost surely. }
\end{aligned}
$$

We define

$$
\rho_{\kappa}^{+}(\omega, t, z, \xi):=\alpha_{\kappa}(z) \rho^{+}(\omega, t, z, \xi)\left|h_{\kappa}(z)\right|^{1 / 2}
$$

and

$$
\rho_{0, \kappa}(\omega, z, \xi):=\alpha_{\kappa}(z) \rho_{0}(\omega, z, \xi)\left|h_{\kappa}(z)\right|^{1 / 2},
$$

with $\omega \in \Omega, t \in[0, T], z \in \tilde{X}_{\kappa} \subset \mathbb{R}^{n}, \xi \in \mathbb{R}$.

Remark 4.1. Most of the time, but not always, we will use the convention of not explicitly writing the chart: for example, writing $\alpha_{\kappa}(z)$ instead of $\left.\alpha_{\kappa}\left(\kappa^{-1}(z)\right)\right)$. Furthermore, we write $\left|h_{\kappa}(z)\right|^{1 / 2}$ (instead of $|h(z)|^{1 / 2}$ ) to remind us that it is a local expression on $X_{\kappa}$, and not a global one on $M$. In other words,

$$
\tilde{X}_{\kappa} \ni z \mapsto\left|h_{\kappa}(z)\right|^{1 / 2}
$$

is a smooth function, and so is its inverse $\left|h_{\kappa}(z)\right|^{-1 / 2}$. Given a function $v$ compactly supported in $\tilde{X}_{\kappa}$, we can "lift" to $M$ the function $\frac{v}{\left|h_{\kappa}\right|^{1 / 2}}$, obtaining a global function on $M$, compactly supported in $X_{\kappa}$ (outside $X_{\kappa}$ the function is set to zero).

We observe that for fixed $\omega \in \Omega, t \in[0, T], \xi \in \mathbb{R}$,

$$
\operatorname{supp} \rho_{\kappa}^{+}(\omega, t, \cdot, \xi) \subset \kappa\left(\operatorname{supp} \alpha_{\kappa}\right) \subset \subset \tilde{X}_{\kappa} \subset \mathbb{R}^{n}
$$

and

$$
\operatorname{supp} \rho_{0, \kappa}(\omega, \cdot, \xi) \subset \kappa\left(\operatorname{supp} \alpha_{\kappa}\right) \subset \subset \tilde{X}_{\kappa} \subset \mathbb{R}^{n},
$$

and thus they may be seen as global functions on $\mathbb{R}^{n}$.

4.2. Regularization of localized equations. Let $\phi_{1}, \phi_{2}$ be a standard mollifiers on $\mathbb{R}^{n}$ and $\mathbb{R}$, respectively, and define the function

$$
\phi_{\varepsilon}(z, \xi):=\varepsilon^{-n} \phi_{1}\left(\frac{z}{\varepsilon}\right) \varepsilon^{-1} \phi_{2}\left(\frac{\xi}{\varepsilon}\right), \quad z \in \mathbb{R}^{n}, \xi \in \mathbb{R},
$$

whose support is contained in $\overline{B_{\varepsilon}(0)} \times[-\varepsilon, \varepsilon]$. 
We define regularizations of $\rho_{\kappa}^{+}$and $\rho_{0, \kappa}$. For $\omega \in \Omega, t \in[0, T], z \in \mathbb{R}^{n}, \xi \in \mathbb{R}$,

$$
\begin{aligned}
& \left(\rho_{\kappa}^{+}\right)_{\varepsilon}(\omega, t)(z, \xi) \\
& \quad:=\int_{\mathbb{R}^{n} \times \mathbb{R}} \rho_{\kappa}^{+}(\omega, t, \bar{z}, \bar{\xi}) \varepsilon^{-n} \phi_{1}\left(\frac{z-\bar{z}}{\varepsilon}\right) \varepsilon^{-1} \phi_{2}\left(\frac{\xi-\bar{\xi}}{\varepsilon}\right) d \bar{\xi} d \bar{z} \\
& \quad=\int_{\mathbb{R}^{n} \times \mathbb{R}} \alpha_{\kappa}(\bar{z}) \rho^{+}(\omega, t, \bar{z}, \bar{\xi})\left|h_{\kappa}(\bar{z})\right|^{1 / 2} \varepsilon^{-n} \phi_{1}\left(\frac{z-\bar{z}}{\varepsilon}\right) \varepsilon^{-1} \phi_{2}\left(\frac{\xi-\bar{\xi}}{\varepsilon}\right) d \bar{\xi} d \bar{z}, \\
& \left(\rho_{0, \kappa}\right)_{\varepsilon}(\omega)(z, \xi) \\
& \quad:=\int_{\mathbb{R}^{n} \times \mathbb{R}} \rho_{0, \kappa}(\omega, \bar{z}, \bar{\xi}) \varepsilon^{-n} \phi_{1}\left(\frac{z-\bar{z}}{\varepsilon}\right) \varepsilon^{-1} \phi_{2}\left(\frac{\xi-\bar{\xi}}{\varepsilon}\right) d \bar{\xi} d \bar{z} \\
& =\int_{\mathbb{R}^{n} \times \mathbb{R}} \alpha_{\kappa}(\bar{z}) \rho_{0}(\omega, \bar{z}, \bar{\xi})\left|h_{\kappa}(\bar{z})\right|^{1 / 2} \varepsilon^{-n} \phi_{1}\left(\frac{z-\bar{z}}{\varepsilon}\right) \varepsilon^{-1} \phi_{2}\left(\frac{\xi-\bar{\xi}}{\varepsilon}\right) d \bar{\xi} d \bar{z} .
\end{aligned}
$$

We set $\varepsilon_{\kappa}:=\operatorname{dist}\left(\kappa\left(\operatorname{supp} \alpha_{\kappa}\right), \partial \tilde{X}_{\kappa}\right)>0$

The main properties of $\left(\rho_{\kappa}^{+}\right)_{\varepsilon}$ and $\left(\rho_{0, \kappa}\right)_{\varepsilon}$ are listed in

Lemma 4.2. Let $\kappa \in \mathcal{A}$. Then

(1) $\left(\rho_{\kappa}^{+}\right)_{\varepsilon}(\omega, t) \in C^{\infty}\left(\mathbb{R}_{z}^{n} \times \mathbb{R}_{\xi}\right)$, for all $(\omega, t) \in \Omega_{T}$.

(2) for $\varepsilon<\varepsilon_{\kappa}$ and for any $\omega \in \Omega, t \in[0, T], \xi \in \mathbb{R}$,

$$
\operatorname{supp}\left(\rho_{\kappa}^{+}\right)_{\varepsilon}(\omega, t)(\cdot, \xi) \subset \kappa\left(\operatorname{supp} \alpha_{\kappa}\right)+\overline{B_{\varepsilon}(0)} \subset \subset \tilde{X}_{\kappa}
$$

This implies in particular that for any $(\omega, t) \in \Omega_{T}$, the function $\left(\rho_{\kappa}^{+}\right)_{\varepsilon}(\omega, t)$ can be seen as an element of $C^{\infty}(M \times \mathbb{R})$, provided that we set it equal to zero outside $X_{\kappa} \times \mathbb{R}$.

(3) $\lim _{\varepsilon \rightarrow 0}\left(\rho_{\kappa}^{+}\right)_{\varepsilon}(\omega, t)(\cdot, \cdot)=\rho_{\kappa}^{+}(\omega, t, \cdot, \cdot)$ in $\mathcal{D}^{\prime}\left(\mathbb{R}^{n} \times \mathbb{R}\right)$ for any $(\omega, t) \in \Omega_{T}$. In particular, for $\psi \in \mathcal{D}\left(X_{\kappa} \times \mathbb{R}\right)$ we have

$$
\begin{aligned}
\int_{M \times \mathbb{R}} \psi(x, \xi) & \frac{\left(\rho_{\kappa}^{+}\right)_{\varepsilon}(\omega, t)(x, \xi)}{\left|h_{\kappa}(x)\right|^{1 / 2}} d \xi d V_{h}(x) \\
& \stackrel{\varepsilon \downarrow 0}{\longrightarrow} \int_{M \times \mathbb{R}} \psi(x, \xi) \alpha_{\kappa}(x) \rho^{+}(\omega, t, x, \xi) d \xi d V_{h}(x),
\end{aligned}
$$

which holds for $\psi \in \mathcal{D}(M \times \mathbb{R})$ as well, because the functions $\frac{\left(\rho_{\kappa}^{+}\right)_{\varepsilon}}{\left|h_{\kappa}\right|^{1 / 2}}$ and $\alpha_{\kappa} \rho^{+}$are supported in $X_{\kappa} \times \mathbb{R}$.

(4) For any $1 \leq p<\infty$ and $(\omega, t) \in \Omega_{T}$,

$$
\frac{\left(\rho_{\kappa}^{+}\right)_{\varepsilon}(\omega, t)(\cdot, \cdot)}{\left|h_{\kappa}(\cdot)\right|^{1 / 2}} \stackrel{\varepsilon \downarrow 0}{\longrightarrow} \alpha_{\kappa}(\cdot) \rho^{+}(\omega, t, \cdot, \cdot) \quad \text { in } L_{\mathrm{loc}}^{p}(M \times \mathbb{R}) .
$$

The listed properties hold true for $\left(\rho_{0, \kappa}\right)_{\varepsilon}$ as well.

Proof. Claims (1) and (2) follow from standard properties of convolution. Claim (3) is an easy consequence of $(2)$ and convergence properties of convolution. To show $L^{p}$-convergence, we argue like this: for any $L>0$, using on $X_{\kappa}$ the coordinates 
given by $\kappa$, we obtain

$$
\begin{aligned}
& \int_{M \times[-L, L]} \mid \frac{\left(\rho_{\kappa}^{+}\right)_{\varepsilon}(\omega, t)(x, \xi)}{\left|h_{\kappa}(x)\right|^{1 / 2}-\alpha_{\kappa}(x) \rho^{+}(\omega, t, x, \xi) \mid} d \xi d V_{h}(x) \\
& =\int_{X_{\kappa} \times[-L, L]}\left|\frac{\left(\rho_{\kappa}^{+}\right)_{\varepsilon}(\omega, t)(x, \xi)}{\left|h_{\kappa}(x)\right|^{1 / 2}}-\alpha_{\kappa}(x) \rho^{+}(\omega, t, x, \xi)\right|^{p} d \xi d V_{h}(x) \\
& =\int_{\tilde{X}_{\kappa} \times[-L, L]}\left|\frac{\left(\rho_{\kappa}^{+}\right)_{\varepsilon}(\omega, t)(z, \xi)}{\left|h_{\kappa}(z)\right|^{1 / 2}}-\alpha_{\kappa}(z) \rho^{+}(\omega, t, z, \xi)\right|^{p}\left|h_{\kappa}(z)\right|^{1 / 2} d \xi d z \\
& =\int_{\tilde{X}_{\kappa} \times[-L, L]}\left|\left(\rho_{\kappa}^{+}\right)_{\varepsilon}(\omega, t)(z, \xi)-\rho_{\kappa}^{+}(\omega, t, z, \xi)\right|^{p}\left|h_{\kappa}(z)\right|^{\frac{1}{2}(1-p)} d \xi d z \\
& \leq C(\mathcal{A}, h, p) \int_{\tilde{X}_{\kappa} \times[-L, L]}\left|\left(\rho_{\kappa}^{+}\right)_{\varepsilon}(\omega, t)(z, \xi)-\rho_{\kappa}^{+}(\omega, t, z, \xi)\right|^{p} d \xi d z .
\end{aligned}
$$

The last integral converges to zero as $\varepsilon$ goes to zero by standard properties of convolution, since $\rho_{\kappa}^{+}(\omega, t, \cdot, \cdot)$ is in $L^{\infty}\left(\mathbb{R}_{z}^{n} \times \mathbb{R}_{\xi}\right)$.

For $(\omega, t) \in \Omega_{T}$ we define the following finite Borel measure on $[0, t] \times M \times \mathbb{R}$ :

$$
C_{b}^{0}([0, t] \times M \times \mathbb{R}) \ni \phi \mapsto \int_{[0, t] \times M \times \mathbb{R}} \phi(s, x, \xi) \alpha_{\kappa}(x) m(d s, d x, d \xi),
$$

denoted by $\left(\alpha_{\kappa} m\right)(\omega, t)$. By definition, $\operatorname{supp}\left(\left(\alpha_{\kappa} m\right)(\omega, t)\right) \subset[0, t] \times \operatorname{supp}\left(\alpha_{\kappa}\right) \times \mathbb{R}$. We define its pushforward $\left(\alpha_{\kappa} m\right)_{\sharp}(\omega, t)$ via the homeomorphism

$$
K:[0, t] \times X_{\kappa} \times \mathbb{R} \rightarrow[0, t] \times \tilde{X}_{k} \times \mathbb{R}, \quad(s, x, \xi) \mapsto(s, \kappa(x), \xi) .
$$

Hence, its action is given by

$$
C_{b}^{0}\left([0, t] \times \tilde{X}_{\kappa} \times \mathbb{R}\right) \ni \phi \mapsto \int_{[0, t] \times \tilde{X}_{\kappa} \times \mathbb{R}} \phi(s, z, \xi) \alpha_{\kappa}(z) m_{\sharp}(d s, d z, d \xi),
$$

where $m_{\sharp}$ is the pushforward of $m$ via $K$. With a little abuse of notation, we will also write $\left(\alpha_{\kappa} m\right)_{\sharp}(\omega, t)$ for the finite Borel on $\tilde{X}_{\kappa} \times \mathbb{R}$ determined by

$$
C_{b}^{0}\left(\tilde{X}_{\kappa} \times \mathbb{R}\right) \ni \phi \mapsto \int_{[0, t] \times \tilde{X}_{\kappa} \times \mathbb{R}} \phi(z, \xi) \alpha_{\kappa}(z) m_{\sharp}(d s, d z, d \xi),
$$

Consequently, $\left(\alpha_{\kappa} m\right)_{\sharp}(\omega, t)$ is a finite Borel measure on $\tilde{X}_{\kappa} \times \mathbb{R}$ that is supported in $\kappa\left(\operatorname{supp} \alpha_{\kappa}\right) \times \mathbb{R} \subset \tilde{X}_{\kappa} \times \mathbb{R}$, and thus it may be naturally viewed as a finite Borel measure on $\mathbb{R}^{n} \times \mathbb{R}$.

We regularize $\left(\alpha_{\kappa} m\right)_{\sharp}$ using the mollifier $\phi_{\varepsilon}$ : for $\omega \in \Omega, t \in[0, T], z \in \mathbb{R}^{n}, \xi \in \mathbb{R}$ we define

$$
\begin{aligned}
\left(\left(\alpha_{\kappa} m\right)_{\sharp}\right)_{\varepsilon}(\omega, t)(z, \xi) & :=\left(\alpha_{\kappa} m\right)_{\sharp}(\omega, t)\left(\varepsilon^{-n} \phi_{1}\left(\frac{z-\cdot}{\varepsilon}\right) \varepsilon^{-1} \phi_{2}\left(\frac{\xi-\cdot}{\varepsilon}\right)\right) \\
& =\int_{[0, t] \times \tilde{X}_{\kappa} \times \mathbb{R}} \phi_{\varepsilon}(z-\bar{z}, \xi-\bar{\xi}) \alpha_{\kappa}(\bar{z}) m_{\sharp}(d s, d \bar{z}, d \bar{\xi}) .
\end{aligned}
$$

The main properties of $\left(\left(\alpha_{\kappa} m\right)_{\sharp}\right)_{\varepsilon}(\omega, t)$ are listed in

Lemma 4.3. Let $\kappa \in \mathcal{A}$. Then

(1) $\left(\left(\alpha_{\kappa} m\right)_{\sharp}\right)_{\varepsilon}(\omega, t) \in C^{\infty}\left(\mathbb{R}_{z}^{n} \times \mathbb{R}_{\xi}\right)$ for all $(\omega, t) \in \Omega_{T}$.

(2) for $\varepsilon<\varepsilon_{\kappa}$ and any $\omega \in \Omega, t \in[0, T], \xi \in \mathbb{R}$.

$$
\operatorname{supp}\left(\left(\left(\alpha_{\kappa} m\right)_{\sharp}\right)_{\varepsilon}(\omega, t)(\cdot, \xi)\right) \subset \kappa\left(\operatorname{supp} \alpha_{\kappa}\right)+\overline{B_{\varepsilon}(0)} \subset \subset \tilde{X}_{\kappa} .
$$

This entails that for fixed $(\omega, t) \in \Omega_{T}$, the function $\left(\left(\alpha_{\kappa} m\right)_{\sharp}\right)_{\varepsilon}(\omega, t)$ can be seen as an element of $C^{\infty}(M \times \mathbb{R})$, provided we set it to zero outside $X_{\kappa} \times \mathbb{R}$. 
(3) $\lim _{\varepsilon \rightarrow 0}\left(\left(\alpha_{\kappa} m\right)_{\sharp}\right)_{\varepsilon}(\omega, t)=\left(\alpha_{\kappa} m\right)_{\sharp}(\omega, t)$ in the sense of measures for any $(\omega, t) \in \Omega_{T}$. In particular, for $\psi \in C_{c}^{0}\left(X_{\kappa} \times \mathbb{R}\right)$,

$$
\begin{aligned}
\int_{M \times \mathbb{R}} \psi(x, \xi) & \frac{\left(\left(\alpha_{\kappa} m\right)_{\sharp}\right)_{\varepsilon}(\omega, t)(x, \xi)}{\left|h_{\kappa}(x)\right|^{1 / 2}} d \xi d V_{h}(x) \\
& \stackrel{\varepsilon \downarrow 0}{\longrightarrow} \int_{M \times \mathbb{R}} \psi(x, \xi)\left(\alpha_{\kappa} m\right)(\omega, t)(d x, d \xi),
\end{aligned}
$$

which is equal to

$$
\int_{[0, t] \times M \times \mathbb{R}} \psi(x, \xi) \alpha_{\kappa}(x) m(d s, d x, d \xi) .
$$

This result holds for $\psi \in C_{c}^{0}(M \times \mathbb{R})$ as well, because the function $\frac{\left(\left(\alpha_{\kappa} m\right)_{\sharp}\right)_{\varepsilon}}{\left|h_{\kappa}\right|^{1 / 2}}$ and the measure $\left(\alpha_{\kappa} m\right)(\omega, t)$ are supported in $X_{\kappa} \times \mathbb{R}$.

(4) for any $(\omega, t) \in \Omega_{T}$ and $\psi \in C_{c}^{1}(M \times \mathbb{R})$,

$$
\begin{aligned}
& \mid \int_{M \times \mathbb{R}} \psi(x, \xi) \partial_{\xi} {\left[\frac{\left(\left(\alpha_{\kappa} m\right)_{\sharp}\right)_{\varepsilon}(\omega, t)(x, \xi)}{\left|h_{\kappa}(x)\right|^{1 / 2}}\right] d \xi d V_{h}(x) \mid } \\
& \leq\left\|\partial_{\xi} \psi\right\|_{L^{\infty}(M \times \mathbb{R})} \int_{[0, t] \times M \times \mathbb{R}} \alpha_{\kappa}(x) m(d s, d x, d \xi) .
\end{aligned}
$$

Proof. The proof is identical to the proof of Lemma 4.2, except for the last point. Let $\psi \in C_{c}^{1}\left(X_{\kappa} \times \mathbb{R}\right)$. Using on $X_{\kappa}$ the coordinates given by $\kappa$, it follows that

$$
\begin{aligned}
-\int_{M \times \mathbb{R}} & \psi(x, \xi) \partial_{\xi}\left[\frac{\left(\left(\alpha_{\kappa} m\right)_{\sharp}\right)_{\varepsilon}(\omega, t)(x, \xi)}{\left|h_{\kappa}(x)\right|^{1 / 2}}\right] d \xi d V_{h}(x) \\
& =\int_{M \times \mathbb{R}} \partial_{\xi} \psi(x, \xi) \frac{\left(\left(\alpha_{\kappa} m\right)_{\sharp}\right)_{\varepsilon}(\omega, t)(x, \xi)}{\left|h_{\kappa}(x)\right|^{1 / 2}} d \xi d V_{h}(x) \\
& =\int_{\tilde{X}_{\kappa} \times \mathbb{R}} \partial_{\xi} \psi(z, \xi)\left(\left(\alpha_{\kappa} m\right)_{\sharp}\right)_{\varepsilon}(\omega, t)(z, \xi) d \xi d z \\
& =\left(\alpha_{\kappa} m\right)_{\sharp}\left(\omega, t\left(\left(\partial_{\xi} \psi\right)_{\varepsilon}\right),\right.
\end{aligned}
$$

where $\left(\partial_{\xi} \psi\right)_{\varepsilon}$ is the convolution of $\partial_{\xi} \psi$ with $\phi_{\varepsilon}$. By basic estimates for convolution, $\left\|\left(\partial_{\xi} \psi\right)_{\varepsilon}\right\|_{L^{\infty}\left(\tilde{X}_{\kappa} \times \mathbb{R}\right)} \leq\left\|\partial_{\xi} \psi\right\|_{L^{\infty}\left(\tilde{X}_{\kappa} \times \mathbb{R}\right)} \leq\left\|\partial_{\xi} \psi\right\|_{L^{\infty}(M \times \mathbb{R})}$, where we observe that $\partial_{\xi} \psi$ can be unambiguously defined on all of $M \times \mathbb{R}$. Hence, we obtain, by the definition of pushforward,

$$
\begin{aligned}
& \mid \int_{M \times \mathbb{R}} \psi(x, \xi) \partial_{\xi} {\left[\frac{\left(\left(\alpha_{\kappa} m\right)_{\sharp}\right)_{\varepsilon}(\omega, t)(x, \xi)}{\left|h_{\kappa}(x)\right|^{1 / 2}}\right] d \xi d V_{h}(x) \mid } \\
& \leq\left\|\partial_{\xi} \psi\right\|_{L^{\infty}(M \times \mathbb{R})} \int_{\tilde{X}_{\kappa} \times \mathbb{R}}\left(\alpha_{\kappa} m\right)_{\sharp}(\omega, t)(d z, d \xi) \\
& \leq\left\|\partial_{\xi} \psi\right\|_{L^{\infty}(M \times \mathbb{R})} \int_{[0, t] \times M \times \mathbb{R}} \alpha_{\kappa}(x) m(d s, d x, d \xi) .
\end{aligned}
$$

In view of the compactness of the supports, the last estimate holds for any $\psi \in$ $C_{c}^{1}(M \times \mathbb{R})$, and the lemma is therefore proved.

To regularize 4.1], we have to consider the following map:

$$
\begin{aligned}
& \nu_{\kappa}: \Omega \times[0, T] \times \tilde{X}_{\kappa} \rightarrow\{\text { finite Borel measures on } \mathbb{R}\}, \\
& \left(\nu_{\kappa}\right)_{\omega, t, z}(\cdot):=\alpha_{\kappa}(z)\left|h_{\kappa}(z)\right|^{1 / 2} \nu_{\omega, t, \kappa^{-1}(z)}(\cdot) .
\end{aligned}
$$


Since, if $z \notin \kappa\left(\operatorname{supp} \alpha_{\kappa}\right)$, then $\left(\nu_{\kappa}\right)_{\omega, t, z}$ is the null measure on $\mathbb{R}$, we can extend $\left(\nu_{\kappa}\right)$ on $\Omega \times[0, T] \times \mathbb{R}^{n}$ in a natural way. This map may be transformed into a Radon measure on $\mathbb{R}^{n} \times \mathbb{R}$ as follows: for all $(\omega, t) \in \Omega_{T}$ and $\psi \in C_{c}^{0}\left(\mathbb{R}^{n} \times \mathbb{R}\right)$, set

$$
\begin{aligned}
\left(\nu_{\kappa}\right)_{\omega, t}(\psi) & :=\int_{\mathbb{R}^{n}}\left(\nu_{\kappa}\right)_{\omega, t, z}(\psi(z, \cdot)) d z \\
& =\int_{\kappa\left(\operatorname{supp} \alpha_{\kappa}\right)} \alpha_{\kappa}(z)\left|h_{\kappa}(z)\right|^{1 / 2}\left(\int_{\mathbb{R}} \psi(z, \xi) \nu_{\omega, t, \kappa^{-1}(z)}(d \xi)\right) d z .
\end{aligned}
$$

The support of this measure is contained in $\kappa\left(\operatorname{supp} \alpha_{\kappa}\right) \times \mathbb{R}$. Once again, we regularize $\left(\nu_{\kappa}\right)_{\omega, t}$ using the mollifier $\phi_{\varepsilon}$. For $(\omega, t) \in \Omega_{T},(z, \xi) \in \mathbb{R}^{n} \times \mathbb{R}$, we set

$$
\left(\nu_{\kappa}\right)_{\varepsilon}(\omega, t)(z, \xi):=\left(\nu_{\kappa}\right)_{\omega, t}\left(\varepsilon^{-n} \phi_{1}\left(\frac{z-\cdot}{\varepsilon}\right) \varepsilon^{-1} \phi_{2}\left(\frac{\xi-\cdot}{\varepsilon}\right)\right) .
$$

The main properties of $\left(\nu_{\kappa}\right)_{\varepsilon}$ are listed in

Lemma 4.4. Let $\kappa \in \mathcal{A}$. Then

(1) $\left(\nu_{\kappa}\right)_{\varepsilon}(\omega, t) \in C^{\infty}\left(\mathbb{R}_{z}^{n} \times \mathbb{R}_{\xi}\right)$ for all $(\omega, t) \in \Omega_{T}$.

(2) for $\varepsilon<\varepsilon_{\kappa}$ and any $\omega \in \Omega, t \in[0, T], \xi \in \mathbb{R}$,

$$
\operatorname{supp}\left(\left(\nu_{\kappa}\right)_{\varepsilon}(\omega, t)(\cdot, \xi)\right) \subset \kappa\left(\operatorname{supp} \alpha_{\kappa}\right)+\overline{B_{\varepsilon}(0)} \subset \subset \tilde{X}_{\kappa} .
$$

Hence, for fixed $(\omega, t) \in \Omega_{T}$, the function $\left(\nu_{\kappa}\right)_{\varepsilon}(\omega, t)$ can be seen as an element of $C^{\infty}(M \times \mathbb{R})$, setting it to zero outside of $X_{\kappa} \times \mathbb{R}$.

(3) $\lim _{\varepsilon \rightarrow 0}\left(\nu_{\kappa}\right)_{\varepsilon}(\omega, t)=\left(\nu_{\kappa}\right)_{\omega, t}$ in the sense of measures for any $(\omega, t) \in \Omega_{T}$. In particular, for $\psi \in C_{c}^{0}\left(X_{\kappa} \times \mathbb{R}\right)$,

$$
\begin{aligned}
\int_{M \times \mathbb{R}} \psi(x, \xi) & \frac{\left(\nu_{\kappa}\right)_{\varepsilon}(\omega, t)(x, \xi)}{\left|h_{\kappa}(x)\right|^{1 / 2}} d \xi d V_{h}(x) \\
& \stackrel{\varepsilon \downarrow 0}{\longrightarrow} \int_{M} \alpha_{\kappa}(x)\left(\int_{\mathbb{R}} \psi(x, \xi) \nu_{\omega, t, x}(d \xi)\right) d V_{h}(x) .
\end{aligned}
$$

This result holds for $\psi \in C_{c}^{0}(M \times \mathbb{R})$ as well, because the function $\frac{\left(\nu_{\kappa}\right)_{\varepsilon}}{\left|h_{\kappa}\right|^{1 / 2}}$ and the measure $\alpha_{\kappa} \nu d V_{h}$ are supported in $X_{\kappa} \times \mathbb{R}$.

(4) For a.e. $(\omega, t) \in \Omega_{T}$, for all $(x, \xi) \in M \times \mathbb{R}$, and for all $\varepsilon<\varepsilon_{\kappa}$,

where $\left(\rho_{\kappa}^{+}\right)_{\varepsilon}$ is given by Lemma 4.2.

$$
\partial_{\xi}\left[\frac{\left(\rho_{\kappa}^{+}\right)_{\varepsilon}(\omega, t)(x, \xi)}{\left|h_{\kappa}(x)\right|^{1 / 2}}\right]=-\frac{\left(\nu_{\kappa}\right)_{\varepsilon}(\omega, t)(x, \xi)}{\left|h_{\kappa}(x)\right|^{1 / 2}},
$$

Proof. Only the last claim requires a proof. Let $\psi$ be in $\mathcal{D}\left(X_{\kappa} \times \mathbb{R}\right)$. By the definition of a generalized kinetic solution, for $\mathbb{P} \otimes d t$-a.e. $(\omega, t) \in \Omega_{T}$,

$$
\begin{aligned}
\int_{M \times \mathbb{R}} & \alpha_{\kappa}(x) \rho^{+}(\omega, t, x, \xi) \partial_{\xi} \psi(x, \xi) d \xi d V_{h}(x) \\
& =\int_{M} \alpha_{\kappa}(x) \int_{\mathbb{R}} \psi(x, \xi) \nu_{\omega, t, x}(d \xi) d V_{h}(x) .
\end{aligned}
$$

Using the coordinates given by $\kappa$, this means

$$
\begin{aligned}
\int_{\kappa\left(\operatorname{supp} \alpha_{\kappa}\right) \times \mathbb{R}} & \rho_{\kappa}^{+}(\omega, t, z, \xi) \partial_{\xi} \psi(z, \xi) d \xi d z \\
& =\int_{M} \alpha_{\kappa}(z) \int_{\mathbb{R}} \psi(z, \xi) \nu_{\omega, t, \kappa^{-1}(z)}(d \xi)\left|h_{\kappa}(z)\right|^{1 / 2} d z .
\end{aligned}
$$

In other words, for all $\psi \in \mathcal{D}\left(\tilde{X}_{\kappa} \times \mathbb{R}\right)$ and for $\mathbb{P} \otimes d t$-a.e. $(\omega, t) \in \Omega_{T}$,

$$
\int_{\kappa\left(\operatorname{supp} \alpha_{\kappa}\right) \times \mathbb{R}} \rho_{\kappa}^{+}(\omega, t, z, \xi) \partial_{\xi} \psi(z, \xi) d z d \xi=\left(\nu_{\kappa}\right)_{\omega, t}(\psi),
$$


which holds for any $\psi \in \mathcal{D}\left(\mathbb{R}^{n} \times \mathbb{R}\right)$, because of the supports of $\rho_{\kappa}^{+}$and $\left(\nu_{\kappa}\right)_{\omega, t}$.

Therefore, by standard properties of convolution, it follows for all $\varepsilon<\varepsilon_{\kappa}$ and for all $(z, \xi) \in \mathbb{R}^{n} \times \mathbb{R}$ that

$$
\partial_{\xi}\left(\rho_{\kappa}^{+}\right)_{\varepsilon}(\omega, t)(z, \xi)=-\left(\nu_{\kappa}\right)_{\varepsilon}(\omega, t)(z, \xi),
$$

for a.e. $(\omega, t) \in \Omega_{T}$. This property remains true if we divide both sides by $\left|h_{\kappa}(z)\right|^{1 / 2}$. The claim now follows.

As a further step towards the regularization of (4.1), we need to define an additional Radon measure on $\mathbb{R}^{n} \times \mathbb{R}$ :

$$
\left(G^{2} \nu_{\kappa}\right)_{\omega, t}(\psi):=\int_{\kappa\left(\operatorname{supp} \alpha_{\kappa}\right)} \int_{\mathbb{R}} \alpha_{\kappa}(z) G^{2}(z, \xi)\left|h_{\kappa}(z)\right|^{1 / 2} \psi(z, \xi) \nu_{\omega, t, \kappa^{-1}(z)}(d \xi) d z,
$$

with $\psi \in C_{c}^{0}\left(\mathbb{R}^{n} \times \mathbb{R}\right)$ and $G^{2}$ is defined in 2.3). In view of Lemma 2.1, we have $G^{2} \in C^{0}(M \times \mathbb{R})$, and thus, because the support of $\alpha_{\kappa}$ is compact, $\alpha_{\kappa} G^{2}\left|h_{\kappa}\right|^{1 / 2}$ may be seen as a (continuous) Borel function on $\mathbb{R}^{n} \times \mathbb{R}$, if extended to zero outside of $\tilde{X}_{\kappa} \times \mathbb{R}$. We regularize $\left(G^{2} \nu_{\kappa}\right)_{\omega, t}$ by convolution. For $(\omega, t) \in \Omega_{T},(z, \xi) \in \mathbb{R}^{n} \times \mathbb{R}$, we set

$$
\left(G^{2} \nu_{\kappa}\right)_{\varepsilon}(\omega, t)(z, \xi):=\left(G^{2} \nu_{\kappa}\right)_{\omega, t}\left(\varepsilon^{-n} \phi_{1}\left(\frac{z-\cdot}{\varepsilon}\right) \varepsilon^{-1} \phi_{2}\left(\frac{\xi-\cdot}{\varepsilon}\right)\right) .
$$

The main properties are listed in the following lemma, whose proof is identical to those of Lemmas 4.2 and 4.3 .

Lemma 4.5. Let $\kappa \in \mathcal{A}$. Then

(1) $\left(G^{2} \nu_{\kappa}\right)_{\varepsilon}(\omega, t) \in C^{\infty}\left(\mathbb{R}_{z}^{n} \times \mathbb{R}_{\xi}\right)$ for all $(\omega, t) \in \Omega_{T}$

(2) for $\varepsilon<\varepsilon_{\kappa}$ and any $\omega \in \Omega, t \in[0, T], \xi \in \mathbb{R}$,

$$
\operatorname{supp}\left(G^{2} \nu_{\kappa}\right)_{\varepsilon}(\omega, t)(\cdot, \xi) \subset \kappa\left(\operatorname{supp} \alpha_{\kappa}\right)+\overline{B_{\varepsilon}(0)} \subset \subset \tilde{X}_{\kappa} .
$$

Thus, for fixed $(\omega, t) \in \Omega_{T}$, the function $\left(G^{2} \nu_{\kappa}\right)_{\varepsilon}(\omega, t)$ can be seen as an element of $C^{\infty}(M \times \mathbb{R})$, provided it is set to zero outside of $X_{\kappa} \times \mathbb{R}$.

(3) $\lim _{\varepsilon \rightarrow 0}\left(G^{2} \nu_{\kappa}\right)_{\varepsilon}(\omega, t)=\left(G^{2} \nu_{\kappa}\right)_{\omega, t}$ in the sense of measures, for $(\omega, t) \in \Omega_{T}$. In particular, for $\psi \in C_{c}^{0}\left(X_{\kappa} \times \mathbb{R}\right)$,

$$
\begin{aligned}
\int_{M \times \mathbb{R}} \psi(x, \xi) & \frac{\left(G^{2} \nu_{\kappa}\right)_{\varepsilon}(\omega, t)(x, \xi)}{\left|h_{\kappa}(x)\right|^{1 / 2}} d \xi d V_{h}(x) \\
& \stackrel{\varepsilon \downarrow 0}{\longrightarrow} \int_{M} \alpha_{\kappa}(x)\left(\int_{\mathbb{R}} \psi(x, \xi) G^{2}(x, \xi) \nu_{\omega, t, x}(d \xi)\right) d V_{h}(x) .
\end{aligned}
$$

This result holds for any $\psi \in C_{c}^{0}(M \times \mathbb{R})$, since the function $\frac{\left(G^{2} \nu_{\kappa}\right)_{\varepsilon}}{\left|h_{\kappa}\right|^{1 / 2}}$ and the measure $\alpha_{\kappa} G^{2} \nu d V_{h}$ are supported in $X_{\kappa} \times \mathbb{R}$.

(4) for any $(\omega, t) \in \Omega_{T}$ and $\psi \in C_{c}^{1}(M \times \mathbb{R})$,

$$
\begin{aligned}
& \left|\int_{M \times \mathbb{R}} \psi(x, \xi) \partial_{\xi}\left[\frac{\left(G^{2} \nu_{\kappa}\right)_{\varepsilon}(\omega, t)(x, \xi)}{\left|h_{\kappa}(x)\right|^{1 / 2}}\right] d \xi d V_{h}(x)\right| \\
& \quad \leq\left\|\partial_{\xi} \psi\right\|_{L^{\infty}(M \times \mathbb{R})} \int_{M} \alpha_{\kappa}(x)\left(\int_{\mathbb{R}} G^{2}(x, \xi) \nu_{\omega, t, x}(d \xi)\right) d V_{h}(x) .
\end{aligned}
$$

For $(\omega, t) \in \Omega_{T}$, consider the function $A_{\kappa}(\omega, t)(\cdot, \cdot)$ defined as

$$
A_{\kappa}(\omega, t)(z, \xi):= \begin{cases}\rho^{+}(\omega, t, z, \xi)\left(f_{z}^{\prime}(\xi), \nabla \alpha_{\kappa}\right)_{h}\left|h_{\kappa}(z)\right|^{1 / 2}, & (z, \xi) \in \tilde{X}_{\kappa} \times \mathbb{R}, \\ 0, & (z, \xi) \notin \tilde{X}_{\kappa} \times \mathbb{R},\end{cases}
$$

which we regularize using the mollifier $\phi_{\varepsilon}$.

Lemma 4.6. Let $\kappa \in \mathcal{A}$. Then 
(1) $\left(A_{\kappa}\right)_{\varepsilon}(\omega, t) \in C^{\infty}\left(\mathbb{R}_{z}^{n} \times \mathbb{R}_{\xi}\right)$, for all $(\omega, t) \in \Omega_{T}$.

(2) for $\varepsilon<\varepsilon_{\kappa}$ and for any $\omega \in \Omega, t \in[0, T], \xi \in \mathbb{R}$,

$$
\operatorname{supp}\left(A_{\kappa}\right)_{\varepsilon}(\omega, t)(\cdot, \xi) \subset \kappa\left(\operatorname{supp} \alpha_{\kappa}\right)+\overline{B_{\varepsilon}(0)} \subset \subset \tilde{X}_{\kappa} .
$$

This implies in particular that for any $(\omega, t) \in \Omega_{T}$, the function $\left(A_{\kappa}\right)_{\varepsilon}(\omega, t)$ can be seen as an element of $C^{\infty}(M \times \mathbb{R})$, provided that we set it equal to zero outside of $X_{\kappa} \times \mathbb{R}$.

(3) $\lim _{\varepsilon \rightarrow 0}\left(A_{\kappa}\right)_{\varepsilon}(\omega, t)=A_{\kappa}(\omega, t)(\cdot, \cdot)$ in $\mathcal{D}^{\prime}\left(\mathbb{R}^{n} \times \mathbb{R}\right)$ for any $(\omega, t) \in \Omega_{T}$. In particular, for $\psi \in \mathcal{D}\left(X_{\kappa} \times \mathbb{R}\right)$, we have

$$
\begin{aligned}
\int_{M \times \mathbb{R}} \psi(x, \xi) & \frac{\left(A_{\kappa}\right)_{\varepsilon}(\omega, t)(x, \xi)}{\left|h_{\kappa}(x)\right|^{1 / 2}} d \xi d V_{h}(x) \\
& \stackrel{\varepsilon \downarrow 0}{\longrightarrow} \int_{M \times \mathbb{R}} \psi(x, \xi) \rho^{+}(\omega, t, x, \xi)\left(f_{x}^{\prime}(\xi) \nabla \alpha_{\kappa}\right)_{h} d \xi d V_{h}(x) .
\end{aligned}
$$

This result holds for any $\psi \in \mathcal{D}(M \times \mathbb{R})$ as well, since the functions involved are supported in $X_{\kappa} \times \mathbb{R}$.

(4) For any $1 \leq p<\infty$ and $(\omega, t) \in \Omega_{T}$,

$$
\frac{\left(A_{\kappa}\right)_{\varepsilon}(\omega, t)(\cdot, \cdot)}{\left|h_{\kappa}(\cdot)\right|^{1 / 2}} \stackrel{\varepsilon \downarrow 0}{\longrightarrow} \rho^{+}(\omega, t, \cdot, \cdot)\left(f^{\prime}(\cdot), \nabla \alpha_{\kappa}\right)_{h} \quad \text { in } L_{\mathrm{loc}}^{p}(M \times \mathbb{R}) .
$$

Proof. Only claim (4) requires a proof, as the other claims are obvious. From the very definition of $A_{\kappa}(\omega, t)$, it follows, for $(z, \xi) \in \tilde{X}_{\kappa} \times \mathbb{R}$,

$$
\begin{aligned}
\left|A_{\kappa}(\omega, t)(z, \xi)\right| & =\left.\left|\rho^{+}(\omega, t, z, \xi)\left(f_{z}^{\prime}(\xi), \nabla \alpha_{\kappa}\right)_{h}\right| h_{\kappa}(z)\right|^{1 / 2} \mid \\
& \leq C(M, h, \mathcal{A})\left|\left(f_{z}^{\prime}(\xi)\right)^{l} \partial_{z^{l}} \alpha_{\kappa}(z)\right|
\end{aligned}
$$

where $l$ sums over 1 to $n$. Thanks to 2.2 , we infer that $A_{\kappa}(\omega, t) \in L_{\text {loc }}^{p}\left(\mathbb{R}^{n} \times \mathbb{R}\right)$. Hence, arguing as in the proof of Lemma 4.2 , we arrive at claim (4).

We eventually need to define a finite signed Borel measure on $\mathbb{R}^{n} \times \mathbb{R}$ denoted by $\left(g_{k} \nu_{\kappa}\right)_{\omega, t}, k \in \mathbb{N}$, where $g_{k} \in C^{0}(M \times \mathbb{R})$ (see equation 2.3$)$ ). We proceed like this: given $\psi \in C_{b}^{0}\left(\mathbb{R}^{n} \times \mathbb{R}\right)$, after extending $\alpha_{\kappa} g_{k}\left|h_{\kappa}\right|^{1 / 2}$ by zero outside of $\tilde{X}_{\kappa} \times \mathbb{R}$, we estimate the following quantity:

$$
\begin{aligned}
& \int_{\kappa\left(\operatorname{supp} \alpha_{\kappa}\right)} \int_{\mathbb{R}} \alpha_{\kappa}(z)\left|g_{k}(z, \xi)\right|\left|h_{\kappa}(z)\right|^{1 / 2}|\psi(z, \xi)| \nu_{\omega, t, \kappa^{-1}(z)}(d \xi) d z \\
& \leq\|\psi\|_{L^{\infty}} \sqrt{D_{1}} \int_{M} \alpha_{\kappa}(x) \int_{\mathbb{R}}(1+|\xi|) \nu_{\omega, t, x}(d \xi) d V_{h}(x) \\
& \leq\|\psi\|_{L^{\infty}} \sqrt{D_{1}} \int_{M} \int_{\mathbb{R}}(1+|\xi|) \nu_{\omega, t, x}(d \xi) d V_{h}(x),
\end{aligned}
$$

where we have used (2.3). Hence, by the "vanishing at infinity" assumption (3.1), we see that the last quantity is finite for all $(\omega, t) \in F$, where $F \in \mathcal{P},(\mathbb{P} \otimes d t)\left(\overline{F^{c}}\right)=0$. In view of this, we define the finite signed Borel measure $\left(g_{k} \nu_{\kappa}\right)_{\omega, t}$ as the one given by the continuous linear functional

$$
C_{b}^{0}\left(\mathbb{R}^{n} \times \mathbb{R}\right) \ni \psi \mapsto \int_{\mathbb{R}^{n}} \int_{\mathbb{R}} \alpha_{\kappa}(z) g_{k}(z, \xi)\left|h_{\kappa}(z)\right|^{1 / 2} \psi(z, \xi) \nu_{\omega, t, \kappa^{-1}(z)}(d \xi) d z,
$$

if $(\omega, t) \in F$; otherwise, $\left(g_{k} \nu_{\kappa}\right)_{\omega, t}$ is set to be the null measure on $\mathbb{R}^{n} \times \mathbb{R}$. Observe that the supports of these signed measures are contained in $\kappa\left(\operatorname{supp} \alpha_{\kappa}\right) \times \mathbb{R}$.

We regularize these measures with the mollifier $\phi_{\varepsilon}$. For $(\omega, t) \in \Omega_{T},(z, \xi) \in$ $\mathbb{R}^{n} \times \mathbb{R}$, and $k \in \mathbb{N}$, set

$$
\left(g_{k} \nu_{\kappa}\right)_{\varepsilon}(\omega, t)(z, \xi):=\left(g_{k} \nu_{\kappa}\right)_{\omega, t}\left(\varepsilon^{-n} \phi_{1}\left(\frac{z-\cdot}{\varepsilon}\right) \varepsilon^{-1} \phi_{2}\left(\frac{\xi-\cdot}{\varepsilon}\right)\right) .
$$


The following result, whose proof is now obvious, holds:

Lemma 4.7. Let $\kappa \in \mathcal{A}$. Then

(1) $\left(g_{k} \nu_{\kappa}\right)_{\varepsilon}(\omega, t) \in C^{\infty}\left(\mathbb{R}_{z}^{n} \times \mathbb{R}_{\xi}\right)$ for all $(\omega, t) \in \Omega_{T}$.

(2) for $\varepsilon<\varepsilon_{\kappa}$ and any $\omega \in \Omega, t \in[0, T], \xi \in \mathbb{R}$,

$$
\operatorname{supp}\left(\left(g_{k} \nu_{\kappa}\right)_{\varepsilon}(\omega, t)(\cdot, \xi)\right) \subset \kappa\left(\operatorname{supp} \alpha_{\kappa}\right)+\overline{B_{\varepsilon}(0)} \subset \subset \tilde{X}_{\kappa} .
$$

This entails that for fixed $\omega \in \Omega, t \in[0, T]$ the function $\left(g_{k} \nu_{\kappa}\right)_{\varepsilon}(\omega, t)$ can be seen as an element of $C^{\infty}(M \times \mathbb{R})$, provided that it is set equal to zero outside of $\tilde{X}_{\kappa} \times \mathbb{R}$.

(3) $\lim _{\varepsilon \rightarrow 0}\left(g_{k} \nu_{\kappa}\right)_{\varepsilon}(\omega, t)=\left(g_{k} \nu_{\kappa}\right)_{\omega, t}$ in the sense of measures for any $(\omega, t) \in$ $\Omega_{T}$. In particular, for $\psi \in C_{c}^{0}\left(X_{\kappa} \times \mathbb{R}\right)$,

$$
\begin{aligned}
\int_{M \times \mathbb{R}} \psi(x, \xi) & \frac{\left(g_{k} \nu_{\kappa}\right)_{\varepsilon}(\omega, t)(x, \xi)}{\left|h_{\kappa}(x)\right|^{1 / 2}} d \xi d V_{h}(x) \\
& \stackrel{\varepsilon \downarrow 0}{\longrightarrow} \int_{M} \alpha_{\kappa}(x)\left(\int_{\mathbb{R}} \psi(x, \xi) g_{k}(x, \xi) \nu_{\omega, t, x}(d \xi)\right) d V_{h}(x) .
\end{aligned}
$$

This result holds for $\psi \in C_{c}^{0}(M \times \mathbb{R})$ as well, because the function $\frac{\left(g_{k} \nu_{\kappa}\right)_{\varepsilon}}{\left|h_{\kappa}\right|^{1 / 2}}$ and the signed measure $\alpha_{\kappa} g_{k} \nu d V_{h}$ are supported in $X_{\kappa} \times \mathbb{R}$.

In view of these results, 4.1 with $\psi \in C_{c}^{1}\left(X_{\kappa} \times \mathbb{R}\right)$ can be written as

$$
\begin{aligned}
& -\int_{\tilde{X}_{\kappa} \times \mathbb{R}} \rho_{\kappa}^{+}(t) \psi d \bar{\xi} d \bar{z}+\int_{\tilde{X}_{\kappa} \times \mathbb{R}} \rho_{0, \kappa} \psi d \bar{\xi} d \bar{z} \\
& \quad+\int_{0}^{t} \int_{\tilde{X}_{\kappa} \times \mathbb{R}} \rho_{\kappa}^{+}(s)\left(f_{\bar{z}}^{\prime}(\bar{\xi})\right)^{l} \partial_{\bar{z}^{l}} \psi d \bar{\xi} d \bar{z} d s \\
& =-\sum_{k \geq 1} \int_{0}^{t} \int_{\tilde{X}_{\kappa} \times \mathbb{R}} \psi\left(g_{k} \nu_{\kappa}\right)_{\omega, s}(d \bar{z}, d \bar{\xi}) d \beta_{k}(s) \\
& \quad-\frac{1}{2} \int_{0}^{t} \int_{\tilde{X}_{\kappa} \times \mathbb{R}} \partial_{\bar{\xi}} \psi\left(G^{2} \nu_{\kappa}\right)_{\omega, s}(d \bar{z}, d \bar{\xi}) d s \\
& \quad+\int_{\tilde{X}_{\kappa} \times \mathbb{R}} \partial_{\bar{\xi}} \psi\left(\alpha_{\kappa} m\right)_{\sharp}(\omega, t)(d \bar{z}, d \bar{\xi}) \\
& \quad-\int_{0}^{t} \int_{\tilde{X}_{\kappa} \times \mathbb{R}} \psi A_{\kappa}(s) d \bar{z} d \bar{\xi} d s, \quad \mathbb{P} \text {-almost surely, }
\end{aligned}
$$

where $\left(f^{\prime}(\cdot)\right)^{l}$ is the $l$ th component of $f^{\prime}$ in the local coordinates given by $\kappa$.

We set for convenience $\mathcal{U}_{\kappa}:=\kappa\left(\operatorname{supp} \alpha_{\kappa}\right)+B_{\varepsilon_{\kappa} / 2}(0)$ and from now on we will consider only $\varepsilon<\varepsilon_{\kappa} / 4$. Let us introduce the following family of test functions $\psi_{z, \xi, \varepsilon}$ (parametrized by $z \in \tilde{X}_{\kappa}, \xi \in \mathbb{R}$, and $\varepsilon<\varepsilon_{\kappa} / 4$ ):

$$
\psi_{z, \xi, \varepsilon}(\cdot, \cdot):=\left\{\begin{array}{ll}
\phi_{\varepsilon}(z-\cdot, \xi-\cdot), & \text { if } B_{\varepsilon}(z) \cap \partial \tilde{X}_{\kappa}=\emptyset \\
0, & \text { otherwise }
\end{array} .\right.
$$

We observe that these functions may be seen as elements of $C_{c}^{1}\left(X_{\kappa} \times \mathbb{R}\right)$ and that, for fixed $\omega \in \Omega, t \in[0, T]$, and $\xi \in \mathbb{R}$,

$$
\operatorname{supp}\left((\cdots)_{\varepsilon}(\omega, t)(\cdot, \xi)\right) \subset \kappa\left(\operatorname{supp} \alpha_{\kappa}\right)+\overline{B_{\varepsilon}(0)} \subset \subset \mathcal{U}_{\kappa}
$$

where $\cdots$ is any of the objects defined above. Moreover, for $\omega \in \Omega, t \in[0, T], \xi \in \mathbb{R}$, and $z \in \tilde{X}_{\kappa} \backslash \mathcal{U}_{\kappa}$, we have that $(\cdots)_{\varepsilon}(\omega, t)(z, \xi)$ is equal to zero and it coincides with the action of $(\cdots)(\omega, t)$ on the function $\psi_{z, \xi, \varepsilon}$. 
We make use of $\psi_{z, \xi, \varepsilon}$ as test function in 4.2 , resulting in the equation

$$
\begin{aligned}
-\left(\rho_{\kappa}^{+}\right)_{\varepsilon}(\omega, t)(z, \xi)+\left(\rho_{0, \kappa}\right)_{\varepsilon}(\omega)(z, \xi) & \\
& -\int_{0}^{t} \int_{\tilde{X}_{\kappa} \times \mathbb{R}} \rho_{\kappa}^{+}(s)\left(f_{\bar{z}}^{\prime}(\bar{\xi})\right)^{l}\left(\partial_{l} \phi_{\varepsilon}\right)(z-\bar{z}, \xi-\bar{\xi}) d \bar{\xi} d \bar{z} d s \\
=- & \sum_{k \geq 1} \int_{0}^{t}\left(g_{k} \nu_{\kappa}\right)_{\varepsilon}(\omega, s)(z, \xi) d \beta_{k}(s) \\
& +\frac{1}{2} \int_{0}^{t} \partial_{\xi}\left[\left(G^{2} \nu_{\kappa}\right)_{\varepsilon}(\omega, s)(z, \xi)\right] d s-\partial_{\xi}\left[\left(\left(\alpha_{\kappa} m\right)_{\sharp}\right)_{\varepsilon}(\omega, t)(z, \xi)\right] \\
& -\int_{0}^{t}\left(A_{\kappa}\right)_{\varepsilon}(\omega, s)(z, \xi) d s, \quad \mathbb{P} \text {-almost surely, }
\end{aligned}
$$

valid for $\varepsilon<\varepsilon_{\kappa} / 4, \xi \in \mathbb{R}$, and $z \in \tilde{X}_{\kappa}$.

For the transport term involving $f^{\prime}$ we need the following following version of the commutator lemma due to DiPerna and Lions [21]:

Lemma 4.8 (DiPerna-Lions commutator estimate). Fix $\kappa \in \mathcal{A}$, and let $\mathcal{E}$ be the smooth vector field on $\tilde{X}_{\kappa} \times \mathbb{R}$ defined by $\mathcal{E}(\bar{z}, \bar{\xi})=\left(\left(f_{\bar{z}}^{\prime}(\bar{\xi})\right)^{1}, \ldots,\left(f_{\bar{z}}^{\prime}(\bar{\xi})\right)^{n}, 0\right)$. Set

$$
r_{\kappa, \varepsilon}(\omega, s)(z, \xi):=\operatorname{div}_{\mathbb{R}^{n+1}}\left(\rho_{\kappa}^{+}(\omega, s) \mathcal{E}\right)_{\varepsilon}(z, \xi)-\operatorname{div}_{\mathbb{R}^{n+1}}\left(\left(\rho_{\kappa}^{+}\right)_{\varepsilon}(\omega, s) \mathcal{E}\right)(z, \xi),
$$

with $(\omega, s) \in \Omega_{T}, z \in \tilde{X}_{\kappa}$, and $\xi \in \mathbb{R}$. Then $r_{\kappa, \varepsilon} \rightarrow 0$ in $L^{1}\left(\Omega_{T} \times \tilde{X}_{\kappa} \times(-L, L)\right)$ as $\varepsilon \rightarrow 0$, for any $L>0$. Furthermore, for $\varepsilon<\varepsilon_{\kappa}$ and for any $(\omega, s) \in \Omega_{T}$, the function $r_{\kappa, \varepsilon}(\omega, s)$ can be seen as an element of $C^{\infty}(M \times \mathbb{R})$, provided it is set to zero outside of $X_{\kappa} \times \mathbb{R}$.

Proof. By previous observations, for any $(\omega, s) \in \Omega_{T}$ and $\xi \in \mathbb{R}$, the quantity $r_{\kappa, \varepsilon}(\omega, s)(\cdot, \xi)$ is zero on the set $\tilde{X}_{\kappa} \backslash \mathcal{U}_{\kappa}$. Thus, it is sufficient to examine the points $z \in \mathcal{U}_{\kappa}$ only. By setting $w:=(z, \xi)$ and $\bar{w}:=(\bar{z}, \bar{\xi}), r_{\kappa, \varepsilon}$ may be rewritten as

$$
\begin{aligned}
r_{\kappa, \varepsilon}(\omega, s)(z, \xi) & \\
= & \sum_{i=1}^{n+1} \int_{|\bar{z}|<\varepsilon} \rho_{\kappa}^{+}(\omega, s, w-\bar{w}) \mathcal{E}_{i}(w-\bar{w})\left(\partial_{i} \phi_{\varepsilon}\right)(\bar{w}) d \bar{w} \\
& -\left(\nabla_{\mathbb{R}^{n+1}}\left(\rho_{\kappa}^{+}\right)_{\varepsilon} \cdot \mathcal{E}\right)(w)-\left(\rho_{\kappa}^{+}\right)_{\varepsilon}(\omega, s)(w) \operatorname{div}_{\mathbb{R}^{n+1}} \mathcal{E}(w) \\
= & \sum_{i=1}^{n+1} \int_{|\bar{z}|<\varepsilon} \rho_{\kappa}^{+}(\omega, s, w-\bar{w})\left(\partial_{i} \phi_{\varepsilon}\right)(\bar{w})\left[\mathcal{E}_{i}(w-\bar{w})-\mathcal{E}_{i}(w)\right] d \bar{w} \\
= & \sum_{i, j=1}^{n+1} \int_{0}^{1} \int_{|\bar{z}|<\varepsilon}\left[\partial_{j} \mathcal{E}_{i}(w)-\partial_{j} \mathcal{E}_{i}(w-\tau \bar{w})\right] \rho_{\kappa}^{+}(\omega, s, w-\bar{w}) \bar{w}_{j}\left(\partial_{i} \phi_{\varepsilon}\right)(\bar{w}) d \bar{w} d \tau \\
& -\sum_{i, j=1}^{n+1} \partial_{j} \mathcal{E}_{i}(w) \int_{|\bar{z}|<\varepsilon} \rho_{\kappa}^{+}(\omega, s, w-\bar{w}) \bar{w}_{j}\left(\partial_{i} \phi_{\varepsilon}\right)(\bar{w}) d \bar{w} \\
= & : a_{\varepsilon}(\omega, s ; z, \xi)+b_{\varepsilon}(\omega, s ; z, \xi)-\left(\rho_{\kappa}^{+}\right)_{\varepsilon}(\omega, s)(w) \operatorname{div}_{\mathbb{R}^{n+1}} \mathcal{E}(w) .
\end{aligned}
$$

Note that for the vector field $\mathcal{E}$ the indices are put in a low position, so that the Einstein summation convention does not apply. Let us deal with the term $a_{\varepsilon}$ first. 
For $L>0$ we have

$$
\begin{aligned}
& \mathbb{E} \int_{0}^{T} \int_{\mathcal{U}_{\kappa} \times(-L, L)}\left|a_{\varepsilon}\right| d w d s \\
& \leq \sum_{i, j=1}^{n+1} \mathbb{E} \int_{0}^{1} \int_{0}^{T} \int_{\mathcal{U}_{\kappa} \times(-L, L)} \\
& \times \int_{\substack{|\bar{z}|<\varepsilon \\
|\bar{\xi}|<\varepsilon}}\left|\partial_{j} \mathcal{E}_{i}(w)-\partial_{j} \mathcal{E}_{i}(w-\tau \bar{w})\right| C(M, h, \mathcal{A})\left|\bar{w}_{j}\left(\partial_{i} \phi_{\varepsilon}\right)(\bar{w})\right| d \bar{w} d w d s d \tau \\
& \leq C(M, h, \mathcal{A}) \sum_{i, j=1}^{n+1} \mathbb{E} \int_{0}^{1} \int_{0}^{T} \int_{|\bar{z}|<\varepsilon} \\
& \times\left|\bar{w}_{j}\left(\partial_{i} \phi_{\varepsilon}\right)(\bar{w})\right|\left\|\partial_{j} \mathcal{E}_{i}(\cdot)-\partial_{j} \mathcal{E}_{i}(\cdot-\tau \bar{w})\right\|_{L^{1}\left(\mathcal{U}_{\kappa} \times(-L, L)\right)} d \bar{w} d s d \tau \\
& \leq C(M, h, \mathcal{A}) \cdot T \sum_{i, j=1}^{n+1} \int_{0}^{1}\left\|\bar{w}_{j}\left(\partial_{i} \phi_{\varepsilon}\right)(\cdot)\right\|_{L^{1}\left(\mathbb{R}^{n+1}\right)} \\
& \times \sup _{\substack{|\overline{\mid}|<\varepsilon \\
|\bar{\xi}|<\varepsilon}}\left\|\partial_{j} \mathcal{E}_{i}(\cdot)-\partial_{j} \mathcal{E}_{i}(\cdot-\tau \bar{w})\right\|_{L^{1}\left(\mathcal{U}_{\kappa} \times(-L, L)\right)} d \tau \\
& \leq C \sum_{i, j=1}^{n+1} \sup _{\substack{|\bar{z}|<\varepsilon \\
|\bar{\xi}|<\varepsilon}}\left\|\partial_{j} \mathcal{E}_{i}(\cdot)-\partial_{j} \mathcal{E}_{i}(\cdot-\bar{w})\right\|_{L^{1}\left(\mathcal{U}_{\kappa} \times(-L, L)\right)} \stackrel{\varepsilon \downarrow 0}{\longrightarrow} 0,
\end{aligned}
$$

where $C(M, h, \mathcal{A})$ is a positive constant depending on $M, h$, and $\mathcal{A}$. Hence, we have the convergence $a_{\varepsilon} \rightarrow 0$ in $L^{1}\left(\Omega_{T} \times \tilde{X}_{\kappa} \times(-L, L)\right)$.

For the term $b_{\varepsilon}$, by following the proof of [21, Lemma II.1], we immediately infer that $b_{\varepsilon} \rightarrow \rho_{\kappa}^{+} \operatorname{div}_{\mathbb{R}^{n+1}} \mathcal{E}$ in $L^{1}\left(\Omega_{T} \times \tilde{X}_{\kappa} \times(-L, L)\right)$ as $\varepsilon \rightarrow 0$.

It remains to deal with the term $-\left(\rho_{\kappa}^{+}\right)_{\varepsilon}(\omega, s)(w) \operatorname{div}_{\mathbb{R}^{n+1}} \mathcal{E}(w)$. As $\left(\rho_{\kappa}^{+}\right)_{\varepsilon}(\omega, s)(\cdot, \cdot)$ belongs to $L^{\infty}\left(\mathbb{R}^{n} \times \mathbb{R}\right)$ for any $(\omega, s) \in \Omega_{T}$, it follows that

$$
\left\|\left(\rho_{\kappa}^{+}\right)_{\varepsilon}(\omega, s)(\cdot, \cdot)-\rho_{\kappa}^{+}(\omega, s, \cdot, \cdot)\right\|_{L^{1}\left(\tilde{X}_{\kappa} \times(-L, L)\right)} \stackrel{\varepsilon \downarrow 0}{\longrightarrow} 0 .
$$

Furthermore, by basic $L^{p}$ convolution estimates,

$$
\begin{aligned}
\left\|\left(\rho_{\kappa}^{+}\right)_{\varepsilon}(\omega, s)(\cdot, \cdot)\right\|_{L^{1}\left(\tilde{X}_{\kappa} \times(-L, L)\right)} & \leq \mathcal{L}^{n+1}\left(\tilde{X}_{\kappa} \times(-L, L)\right)\left\|\left(\rho_{\kappa}^{+}\right)_{\varepsilon}(\omega, s)(\cdot, \cdot)\right\|_{L^{\infty}\left(\mathbb{R}^{n} \times \mathbb{R}\right)} \\
& \leq \mathcal{L}^{n+1}\left(\tilde{X}_{\kappa} \times(-L, L)\right)\left\|\rho_{\kappa}^{+}(\omega, s, \cdot, \cdot)\right\|_{L^{\infty}\left(\mathbb{R}^{n} \times \mathbb{R}\right)} \\
& \leq C(M, h, \mathcal{A}, L),
\end{aligned}
$$

uniformly in $(\omega, s) \in \Omega_{T}$. Thus, by Hölder's inequality and smoothness of $\mathcal{E}$,

$$
\begin{aligned}
& \mathbb{E} \int_{0}^{T} \int_{\tilde{X}_{\kappa} \times(-L, L)}\left|\operatorname{div}_{\mathbb{R}^{n+1}} \mathcal{E}(z, \xi)\right|\left|\left(\rho_{\kappa}^{+}\right)_{\varepsilon}(\omega, s)(z, \xi)-\rho_{\kappa}^{+}(\omega, s, z, \xi)\right| d \xi d z d s \\
& \leq \mathbb{E} \int_{0}^{T}\left\|\operatorname{div}_{\mathbb{R}^{n+1}} \mathcal{E}\right\|_{L^{\infty}\left(\tilde{X}_{\kappa} \times(-L, L)\right)}\left\|\left(\rho_{\kappa}^{+}\right)_{\varepsilon}(\omega, s)-\rho_{\kappa}^{+}(\omega, s, \cdot, \cdot)\right\|_{L^{1}\left(\tilde{X}_{\kappa} \times(-L, L)\right)} d s \\
& \leq C \mathbb{E} \int_{0}^{T}\left\|\left(\rho_{\kappa}^{+}\right)_{\varepsilon}(\omega, s)-\rho_{\kappa}^{+}(\omega, s, \cdot, \cdot)\right\|_{L^{1}\left(\tilde{X}_{\kappa} \times(-L, L)\right)} d s \stackrel{\varepsilon \downarrow 0}{\longrightarrow} 0,
\end{aligned}
$$

by means of dominated convergence. We conclude that

$$
-\left(\rho_{\kappa}^{+}\right)_{\varepsilon} \operatorname{div}_{\mathbb{R}^{n+1}} \mathcal{E} \stackrel{\varepsilon \downarrow 0}{\longrightarrow}-\rho_{\kappa}^{+} \operatorname{div}_{\mathbb{R}^{n+1}} \mathcal{E} \quad \text { in } L^{1}\left(\Omega_{T} \times \tilde{X}_{\kappa} \times(-L, L)\right),
$$

and thus the desired convergence of $r_{\kappa, \varepsilon}$ to zero follows.

The remaining statements are now obvious and thus the lemma is proved. 
In view of Lemma 4.8 , equation 4.3 becomes

$$
\begin{aligned}
-\left(\rho_{\kappa}^{+}\right)_{\varepsilon}(\omega, t)(z, \xi)+\left(\rho_{0, \kappa}\right)_{\varepsilon}(\omega)(z, \xi) & \\
& -\int_{0}^{t} \operatorname{div}_{\mathbb{R}^{n}}\left(\left(\rho_{\kappa}^{+}\right)_{\varepsilon}(\omega, s)(z, \xi) f_{z}^{\prime}(\xi)\right) d s \\
=- & \sum_{k \geq 1} \int_{0}^{t}\left(g_{k} \nu_{\kappa}\right)_{\varepsilon}(\omega, s)(z, \xi) d \beta_{k}(s) \\
& +\frac{1}{2} \int_{0}^{t} \partial_{\xi}\left[\left(G^{2} \nu_{\kappa}\right)_{\varepsilon}(\omega, s)(z, \xi)\right] d s-\partial_{\xi}\left[\left(\left(\alpha_{\kappa} m\right)_{\sharp}\right)_{\varepsilon}(\omega, t)(z, \xi)\right] \\
& -\int_{0}^{t}\left(A_{\kappa}\right)_{\varepsilon}(\omega, s)(z, \xi) d s+\int_{0}^{t} r_{\kappa, \varepsilon}(\omega, s)(z, \xi) d s, \quad \mathbb{P} \text {-almost surely, }
\end{aligned}
$$

valid for $\varepsilon<\varepsilon_{\kappa} / 4, \xi \in \mathbb{R}$, and $z \in \tilde{X}_{\kappa}$.

We recall that, for fixed $(\omega, s) \in \Omega_{T}$ and $\xi \in \mathbb{R}$, the smooth vector field

$$
\tilde{X}_{\kappa} \ni z \mapsto\left(\rho_{\kappa}^{+}\right)_{\varepsilon}(\omega, s)(z, \xi) f_{z}^{\prime}(\xi) \in \mathbb{R}^{n}
$$

is compactly supported in $\kappa\left(\operatorname{supp} \alpha_{\kappa}\right)+\overline{B_{\varepsilon}(0)} \subset \subset \tilde{X}_{\kappa}$. The same property must be enjoyed by the smooth vector field

$$
\tilde{X}_{\kappa} \ni z \mapsto \frac{\left(\rho_{\kappa}^{+}\right)_{\varepsilon}(\omega, s)(z, \xi) f_{z}^{\prime}(\xi)}{\left|h_{\kappa}(z)\right|^{1 / 2}} \in \mathbb{R}^{n} .
$$

Therefore, for any $(\omega, s) \in \Omega_{T}$ and $\xi \in \mathbb{R}$, we pushforward this vector field to $X_{\kappa}$ via the diffeomorphism $\kappa^{-1}: \tilde{X}_{\kappa} \rightarrow X_{\kappa}$. Because of the compactness of its support, this new vector field may be seen as a global smooth vector field on $M$, if extended to zero outside of its support (cf. [43, Prop. 4.10]):

$$
M \ni x \mapsto \frac{\left(\rho_{\kappa}^{+}\right)_{\varepsilon}(\omega, s)(x, \xi) f_{x}^{\prime}(\xi)}{\left|h_{\kappa}(x)\right|^{1 / 2}} \in T M .
$$

Moreover, in the coordinates given by $\kappa$, it holds

$$
\begin{aligned}
\operatorname{div}_{h}\left(\frac{\left(\rho_{\kappa}^{+}\right)_{\varepsilon}(\omega, s)(x, \xi) f_{x}^{\prime}(\xi)}{\left|h_{\kappa}(x)\right|^{1 / 2}}\right) & =\frac{1}{\left|h_{\kappa}(z)\right|^{1 / 2}} \partial_{z^{l}}\left(\left|h_{\kappa}(z)\right|^{1 / 2} \frac{\left(\rho_{\kappa}^{+}\right)_{\varepsilon}(\omega, s)(z, \xi)\left(f_{z}^{\prime}(\xi)\right)^{l}}{\left|h_{\kappa}(z)\right|^{1 / 2}}\right) \\
& =\frac{1}{\left|h_{\kappa}(z)\right|^{1 / 2}} \operatorname{div}_{\mathbb{R}^{n}}\left(\left(\rho_{\kappa}^{+}\right)_{\varepsilon}(\omega, s)(z, \xi) f_{z}^{\prime}(\xi)\right) \quad \text { in } X_{\kappa},
\end{aligned}
$$

from the very definition of $\operatorname{div}_{h}$.

We divide (4.4) by $\left|h_{\kappa}(z)\right|^{1 / 2}$ and then "pull back" to $M$. The result is

$$
\begin{aligned}
& -\frac{\left(\rho_{\kappa}^{+}\right)_{\varepsilon}(\omega, t)(x, \xi)}{\left|h_{\kappa}(x)\right|^{1 / 2}}+\frac{\left(\rho_{0, \kappa}\right)_{\varepsilon}(\omega)(x, \xi)}{\left|h_{\kappa}(x)\right|^{1 / 2}} \\
& \quad-\int_{0}^{t} \operatorname{div}_{h}\left(\frac{\left(\rho_{\kappa}^{+}\right)_{\varepsilon}(\omega, s)(x, \xi) f_{x}^{\prime}(\xi)}{\left|h_{\kappa}(x)\right|^{1 / 2}}\right) d s \\
& =-\sum_{k \geq 1} \int_{0}^{t} \frac{\left(g_{k} \nu_{\kappa}\right)_{\varepsilon}(\omega, s)(x, \xi)}{\left|h_{\kappa}(x)\right|^{1 / 2}} d \beta_{k}(s) \\
& \quad+\frac{1}{2} \int_{0}^{t} \partial_{\xi}\left[\frac{\left(G^{2} \nu_{\kappa}\right)_{\varepsilon}(\omega, s)(x, \xi)}{\left|h_{\kappa}(x)\right|^{1 / 2}}\right] d s-\partial_{\xi}\left[\frac{\left(\left(\alpha_{\kappa} m\right)_{\sharp}\right)_{\varepsilon}(\omega, t)(x, \xi)}{\left|h_{\kappa}(x)\right|^{1 / 2}}\right] \\
& \quad-\int_{0}^{t} \frac{\left(A_{\kappa}\right)_{\varepsilon}(\omega, s)(x, \xi)}{\left|h_{\kappa}(x)\right|^{1 / 2}} d s+\int_{0}^{t} \frac{r_{\kappa, \varepsilon}(\omega, s)(x, \xi)}{\left|h_{\kappa}(x)\right|^{1 / 2}} d s, \quad \mathbb{P} \text {-almost surely, }
\end{aligned}
$$


which is valid for $\varepsilon<\varepsilon_{\kappa} / 4, \xi \in \mathbb{R}, x \in M$, and $t \in[0, T]$. In some of the terms we have used the relation $\partial_{\xi}(\cdots)\left|h_{\kappa}(x)\right|^{-1 / 2}=\partial_{\xi}\left(\cdots\left|h_{\kappa}(x)\right|^{-1 / 2}\right)$.

In the proof of Lemma 4.12 (for example) we will have to work with certain Radon measures associated to $g_{k}^{2}$, which we define now. For all $k \in \mathbb{N}$, set

$$
\left(g_{k}^{2} \nu_{\kappa}\right)_{\omega, t}(\psi):=\int_{\kappa\left(\operatorname{supp} \alpha_{\kappa}\right)} \int_{\mathbb{R}} \alpha_{\kappa}(z) g_{k}^{2}(z, \xi)\left|h_{\kappa}(z)\right|^{1 / 2} \psi(z, \xi) \nu_{\omega, t, \kappa^{-1}(z)}(d \xi) d z,
$$

with $\psi \in C_{c}^{0}\left(\mathbb{R}^{n} \times \mathbb{R}\right)$. By assumption $g_{k}^{2} \in C^{0}(M \times \mathbb{R})$ and because the support of $\alpha_{\kappa}$ is compact, $\alpha_{\kappa} g_{k}^{2}\left|h_{\kappa}\right|^{1 / 2}$ may be seen as a (continuous) Borel function on $\mathbb{R}^{n} \times \mathbb{R}$, if extended to zero outside of $\tilde{X}_{\kappa} \times \mathbb{R}$.

As always, we regularize these measures using a mollifier. For $(\omega, t) \in \Omega_{T}$ and $(z, \xi) \in \mathbb{R}^{n} \times \mathbb{R}$, set

$$
\left(g_{k}^{2} \nu_{\kappa}\right)_{\varepsilon}(\omega, t)(z, \xi):=\left(g_{k}^{2} \nu_{\kappa}\right)_{\omega, t}\left(\varepsilon^{-n} \phi_{1}\left(\frac{z-\cdot}{\varepsilon}\right) \varepsilon^{-1} \phi_{2}\left(\frac{\xi-\cdot}{\varepsilon}\right)\right) .
$$

The main properties are listed in the following lemma, whose proof is obvious in view of earlier (similar) lemmas.

Lemma 4.9. Let $\kappa \in \mathcal{A}$. Then

(1) $\left(g_{k}^{2} \nu_{\kappa}\right)_{\varepsilon}(\omega, t) \in C^{\infty}\left(\mathbb{R}_{z}^{n} \times \mathbb{R}_{\xi}\right)$ for all $(\omega, t) \in \Omega_{T}$;

(2) for $\varepsilon<\varepsilon_{\kappa}$ and any $\omega \in \Omega, t \in[0, T], \xi \in \mathbb{R}$,

$$
\operatorname{supp}\left(\left(g_{k}^{2} \nu_{\kappa}\right)_{\varepsilon}(\omega, t)(\cdot, \xi)\right) \subset \kappa\left(\operatorname{supp} \alpha_{\kappa}\right)+\overline{B_{\varepsilon}(0)} \subset \subset \tilde{X}_{\kappa} \text {. }
$$

Thus, for fixed $(\omega, t) \in \Omega_{T}$, the function $\left(g_{k}^{2} \nu_{\kappa}\right)_{\varepsilon}(\omega, t)$ can be seen as an element of $C^{\infty}(M \times \mathbb{R})$, provided it is set zero outside of $X_{\kappa} \times \mathbb{R}$.

(3) $\lim _{\varepsilon \rightarrow 0}\left(g_{k}^{2} \nu_{\kappa}\right)_{\varepsilon}(\omega, t)=\left(g_{k}^{2} \nu_{\kappa}\right)_{\omega, t}$ in the sense of measures for any $(\omega, t) \in$ $\Omega_{T}$. In particular, for $\psi \in C_{c}^{0}\left(X_{\kappa} \times \mathbb{R}\right)$,

$$
\begin{aligned}
\int_{M \times \mathbb{R}} \psi(x, \xi) & \frac{\left(g_{k}^{2} \nu_{\kappa}\right)_{\varepsilon}(\omega, t)(x, \xi)}{\left|h_{\kappa}(x)\right|^{1 / 2}} d \xi d V_{h}(x) \\
& \stackrel{\varepsilon \downarrow 0}{\longrightarrow} \int_{M} \alpha_{\kappa}(x)\left(\int_{\mathbb{R}} \psi(x, \xi) g_{k}^{2}(x, \xi) \nu_{\omega, t, x}(d \xi)\right) d V_{h}(x) .
\end{aligned}
$$

This result holds for $\psi \in C_{c}^{0}(M \times \mathbb{R})$ as well, since the function $\frac{\left(g_{k}^{2} \nu_{\kappa}\right)_{\varepsilon}}{\left|h_{\kappa}\right|^{1 / 2}}$ and the measure $\alpha_{\kappa} g_{k}^{2} \nu d V_{h}$ are supported in $X_{\kappa} \times \mathbb{R}$.

We need to define the following functions, for $(\omega, t, x, \xi) \in \Omega \times[0, T] \times M \times \mathbb{R}$ and $\varepsilon<\bar{\varepsilon}:=\frac{1}{4} \min _{\kappa \in \mathcal{A}}\left\{\varepsilon_{\kappa}\right\}$ :

$$
\begin{aligned}
& \Gamma_{\varepsilon}(\omega, t)(x, \xi):=\sum_{\kappa \in \mathcal{A}} \frac{\Gamma_{\kappa, \varepsilon}(\omega, t)(x, \xi)}{\left|h_{\kappa}(x)\right|^{1 / 2}}, \\
& \Gamma_{\varepsilon}=\rho_{\varepsilon}^{+}, \rho_{0, \varepsilon},\left(g_{k} \nu\right)_{\varepsilon},\left(G^{2} \nu\right)_{\varepsilon},\left(g_{k}^{2} \nu\right)_{\varepsilon}, m_{\varepsilon}, A_{\varepsilon}, r_{\varepsilon}, \nu_{\varepsilon}, \\
& \Gamma_{\kappa, \varepsilon}=\left(\rho_{\kappa}^{+}\right)_{\varepsilon},\left(\rho_{0, \kappa}\right)_{\varepsilon},\left(g_{k} \nu_{\kappa}\right)_{\varepsilon},\left(G^{2} \nu_{\kappa}\right)_{\varepsilon},\left(g_{k}^{2} \nu_{\kappa}\right)_{\varepsilon},\left(\left(\alpha_{\kappa} m\right)_{\sharp}\right)_{\varepsilon},\left(A_{\kappa}\right)_{\varepsilon}, r_{\kappa, \varepsilon},\left(\nu_{\kappa}\right)_{\varepsilon} .
\end{aligned}
$$

The properties of these functions are listed in

Lemma 4.10. For $\varepsilon<\bar{\varepsilon}$ and $(\omega, t) \in \Omega_{T}$, the functions defined above belong to $C^{\infty}(M \times \mathbb{R})$. Furthermore, for $(\omega, t) \in \Omega_{T}$, the following convergence hold as $\varepsilon \rightarrow 0$ :

(1) $\rho_{\varepsilon}^{+}(\omega, t) \rightarrow \rho^{+}(\omega, t)$ in $L_{\mathrm{loc}}^{p}(M \times \mathbb{R})$, for all $1 \leq p<\infty$.

(2) $\rho_{0, \varepsilon}(\omega) \rightarrow \rho_{0}(\omega)$ in $L_{\text {loc }}^{p}(M \times \mathbb{R})$, for all $1 \leq p<\infty$.

(3) $\left(g_{k} \nu\right)_{\varepsilon}(\omega, t) \rightarrow g_{k} \nu_{\omega, t} d V_{h}$ in the sense of measures.

(4) $\left(G^{2} \nu\right)_{\varepsilon}(\omega, t) \rightarrow G^{2} \nu_{\omega, t} d V_{h}$ in the sense of measures.

(5) $\left(g_{k}^{2} \nu\right)_{\varepsilon}(\omega, t) \rightarrow g_{k}^{2} \nu_{\omega, t} d V_{h}$ in the sense of measures. 
(6) $\left.m_{\varepsilon}(\omega, t) \rightarrow m(\omega)\right|_{[0, t] \times M \times \mathbb{R}}$ in the sense of measures; the restriction of the measure $m(\omega)$ to $[0, t] \times M \times \mathbb{R}$ is denoted by $\left.m(\omega)\right|_{[0, t] \times M \times \mathbb{R}}$.

(7) $A_{\varepsilon}(\omega, t) \rightarrow 0$ in $L_{\mathrm{loc}}^{p}(M \times \mathbb{R})$, for all $1 \leq p<\infty$.

(8) $r_{\varepsilon} \rightarrow 0$ in $L^{1}\left(\Omega_{T} \times M \times(-L, L)\right)$, for all $L>0$.

(9) $\nu_{\varepsilon}(\omega, t) \rightarrow \nu_{\omega, t} d V_{h}$ in the sense of measures; moreover, for a.e. $(\omega, t) \in \Omega_{T}$ and all $(x, \xi) \in M \times \mathbb{R}, \partial_{\xi} \rho_{\varepsilon}^{+}(\omega, t)(x, \xi)=-\nu_{\varepsilon}(\omega, t)(x, \xi)$.

(10) for fixed $\varepsilon<\bar{\varepsilon}$ and any $(\omega, t) \in \Omega_{T}$,

$$
\sum_{k=1}^{p}\left(g_{k}^{2} \nu\right)_{\varepsilon}(\omega, t)(x, \xi) \stackrel{p \rightarrow \infty}{\longrightarrow}\left(G^{2} \nu\right)_{\varepsilon}(\omega, t)(x, \xi), \quad \forall(x, \xi) \in M \times \mathbb{R} .
$$

Proof. The smoothness of the functions as well claims (1) to (6), and (9) are direct consequences of Lemmas 4.2, 4.3, 4.5, 4.6, 4.7. and 4.8, and the fact that the partition of unity is finite.

Furthermore, from Lemma 4.6, the limit for $A_{\varepsilon}(\omega, t)$ is

$$
\sum_{\kappa \in \mathcal{A}} \rho^{+}(\omega, t, \cdot, \cdot)\left(f^{\prime}(\cdot), \nabla \alpha_{\kappa}\right)_{h}=\rho^{+}(\omega, t, \cdot, \cdot)\left(f^{\prime}(\cdot), \nabla 1\right)_{h}=0 .
$$

To verify claim (8), we notice from Lemma 4.8 that

$$
\begin{aligned}
\int_{\Omega_{T} \times M \times(-L, L)} & \frac{\left|r_{\kappa, \varepsilon}(\omega, t)(x, \xi)\right|}{\left|h_{\kappa}(x)\right|^{1 / 2}} d \xi d V_{h}(x) d t \mathbb{P}(d \omega) \\
& =\int_{\Omega_{T} \times(-L, L)} \int_{\tilde{X}_{\kappa}}\left|r_{\kappa, \varepsilon}(\omega, t)(z, \xi)\right| d z d \xi d t \mathbb{P}(d \omega) \stackrel{\varepsilon \downarrow 0}{\longrightarrow} 0 .
\end{aligned}
$$

Summing over $\kappa$, we obtain the desired conclusion.

To prove claim (10), we begin by observing that Lemma 2.1 holds for $G_{p}^{2}(x, \xi):=$ $\sum_{k=1}^{p}\left|g_{k}(x, \xi)\right|^{2}$, namely, $G_{p}^{2}$ is locally Lipschitz, uniformly in $p \in \mathbb{N}$. Hence, by means of the Ascoli-Arzelá theorem, $G_{p}^{2} \rightarrow G^{2}$ uniformly on compact sets. It is therefore clear that for any $\psi \in C_{c}^{0}\left(\mathbb{R}^{n} \times \mathbb{R}\right),(\omega, t) \in \Omega_{T}$, and $\kappa \in \mathcal{A}$, we have

$$
\sum_{k=1}^{p}\left(g_{k}^{2} \nu_{\kappa}\right)_{\omega, t}(\psi) \stackrel{p \rightarrow \infty}{\longrightarrow}\left(G^{2} \nu_{\kappa}\right)_{\omega, t}(\psi)
$$

We conclude hence that, for fixed $\varepsilon<\bar{\varepsilon}$ and $(\omega, t) \in \Omega_{T}$,

$$
\sum_{k=1}^{p}\left(g_{k}^{2} \nu\right)_{\varepsilon}(\omega, t)(\cdot, \cdot) \stackrel{p \rightarrow \infty}{\longrightarrow}\left(G^{2} \nu\right)_{\varepsilon}(\omega, t)(\cdot, \cdot), \quad \text { pointwise on } M \times \mathbb{R} \text {. }
$$

4.3. Global equation and renormalization. To obtain a single (global) equation, we sum over $\kappa$ in (4.5), arriving at

$$
\begin{aligned}
& -\rho_{\varepsilon}^{+}(\omega, t)(x, \xi)+\rho_{0, \varepsilon}(\omega)(x, \xi) \\
& =\int_{0}^{t} \operatorname{div}_{h}\left(\rho_{\varepsilon}^{+}(\omega, s)(x, \xi) f_{x}^{\prime}(\xi)\right) d s \\
& \quad-\sum_{k \geq 1} \int_{0}^{t}\left(g_{k} \nu\right)_{\varepsilon}(\omega, s)(x, \xi) d \beta_{k}(s) \\
& \quad+\frac{1}{2} \int_{0}^{t} \partial_{\xi}\left[\left(G^{2} \nu\right)_{\varepsilon}(\omega, s)(x, \xi)\right] d s-\partial_{\xi}\left[m_{\varepsilon}(\omega, t)(x, \xi)\right] \\
& \quad-\int_{0}^{t} A_{\varepsilon}(\omega, s)(x, \xi) d s+\int_{0}^{t} r_{\varepsilon}(\omega, s)(x, \xi) d s \\
& =:-H_{\varepsilon}(t)(x, \xi)-I_{\varepsilon}(t)(x, \xi), \quad \mathbb{P} \text {-almost surely, }
\end{aligned}
$$


valid for $\varepsilon<\bar{\varepsilon},(x, \xi) \in M \times \mathbb{R}$, and $t \in[0, T]$, where

$$
H_{\varepsilon}(t)(x, \xi):=\sum_{k \geq 1} \int_{0}^{t}\left(g_{k} \nu\right)_{\varepsilon}(\omega, s)(x, \xi) d \beta_{k}(s)
$$

and

$$
\begin{aligned}
I_{\varepsilon}(t)(x, \xi):= & -\int_{0}^{t} \operatorname{div}_{h}\left(\rho_{\varepsilon}^{+}(\omega, s)(x, \xi) f_{x}^{\prime}(\xi)\right) d s-\frac{1}{2} \int_{0}^{t} \partial_{\xi}\left[\left(G^{2} \nu\right)_{\varepsilon}(\omega, s)(x, \xi)\right] d s \\
& +\partial_{\xi}\left[m_{\varepsilon}(\omega, t)(x, \xi)\right]+\int_{0}^{t} A_{\varepsilon}(\omega, s)(x, \xi) d s-\int_{0}^{t} r_{\varepsilon}(\omega, s)(x, \xi) d s
\end{aligned}
$$

Observe that $I_{\varepsilon}(\cdot)(x, \xi)$ is in $B V[0, T]$, càdlàg, and adapted. In fact, we have that $I_{\varepsilon}(\cdot)(x, \xi)-\partial_{\xi}\left[m_{\varepsilon}(\cdot, \cdot)(x, \xi)\right]$ is absolutely continuous.

Applying Itô's formula for semimartingales [56], we obtain the following equation for $\left(\rho_{\varepsilon}^{+}(\cdot, \cdot)(x, \xi)\right)^{2}: \mathbb{P}$-almost surely,

$$
\begin{aligned}
& \left(\rho_{\varepsilon}^{+}(\omega, t)(x, \xi)\right)^{2}=\left(\rho_{0, \varepsilon}(\omega)(x, \xi)\right)^{2}+2 \int_{0}^{t} \rho_{\varepsilon}^{+}\left(\omega, s_{-}\right)(x, \xi) d H_{\varepsilon}(s)(x, \xi) \\
& \quad+2 \int_{0}^{t} \rho_{\varepsilon}^{+}\left(\omega, s_{-}\right)(x, \xi) I_{\varepsilon}(d s)(x, \xi)+\int_{0}^{t} d\left[H_{\varepsilon}(s)(x, \xi)\right] \\
& \quad+\int_{0}^{t} d\left[I_{\varepsilon}(s)(x, \xi)\right]+2 \int_{0}^{t} d\left[H_{\varepsilon}(s)(x, \xi), I_{\varepsilon}(s)(x, \xi)\right] \\
& +\sum_{0<s \leq t}\left\{\left(\rho_{\varepsilon}^{+}(\omega, s)(x, \xi)\right)^{2}-\left(\rho_{\varepsilon}^{+}\left(\omega, s_{-}\right)(x, \xi)\right)^{2}\right. \\
& \left.\quad-2 \rho_{\varepsilon}^{+}\left(\omega, s_{-}\right)(x, \xi) \Delta \rho_{\varepsilon}^{+}(\omega, s)(x, \xi)-\left(\Delta \rho_{\varepsilon}^{+}(\omega, s)(x, \xi)\right)^{2}\right\}
\end{aligned}
$$

valid for $\varepsilon<\bar{\varepsilon},(x, \xi) \in M \times \mathbb{R}$, and $t \in[0, T]$. Here $d[\cdots]$ denotes quadratic covariation, and $\Delta f(s)$ indicates the jump of a function $f$ at $s$, that is, $\Delta f(s)=f(s)-$ $f\left(s_{-}\right)$. We observe that $\sum_{0<s \leq t}\{\cdots\}$ is identically zero, and so is $\int_{0}^{t} d\left[H_{\varepsilon}(s), I_{\varepsilon}(s)\right]$, because $H_{\varepsilon}$ is a continuous martingale and $I_{\varepsilon}$ is of bounded variation. Note that $\int_{0}^{t} d\left[I_{\varepsilon}(s)(x, \xi)\right] \geq 0$. Hence, the equation for $\left(\rho_{\varepsilon}^{+}\right)^{2}$ simplifies to

$$
\begin{aligned}
& \left(\rho_{\varepsilon}^{+}(\omega, t)(x, \xi)\right)^{2}=\left(\rho_{0, \varepsilon}(\omega)(x, \xi)\right)^{2}+2 \int_{0}^{t} \rho_{\varepsilon}^{+}\left(\omega, s_{-}\right)(x, \xi) d H_{\varepsilon}(s)(x, \xi) \\
& \quad+2 \int_{0}^{t} \rho_{\varepsilon}^{+}\left(\omega, s_{-}\right)(x, \xi) I_{\varepsilon}(d s)(x, \xi)+\int_{0}^{t} d\left[H_{\varepsilon}(s)(x, \xi)\right] \\
& \quad+\int_{0}^{t} d\left[I_{\varepsilon}(s)(x, \xi)\right], \quad \mathbb{P} \text {-almost surely. }
\end{aligned}
$$

4.4. Proof of Proposition 4.1. We add 4.7 and 4.6), and multiply the result by $0 \leq \theta \in D(M \times \mathbb{R})$; afterwards, we integrate with respect to $d \xi d V_{h}(x) d \mathbb{P}$. In view of the (stochastic) Fubini theorem, we can interchange integrals as we see fit. Consequently,

$$
\begin{aligned}
& \mathbb{E} \int_{M \times \mathbb{R}} H_{\varepsilon}(t)(x, \xi) \theta d \xi d V_{h}(x)=0, \\
& \mathbb{E} \int_{M \times \mathbb{R}} \theta \int_{0}^{t} \rho_{\varepsilon}^{+}(\omega, s)(x, \xi) d H_{\varepsilon}(s)(x, \xi) d \xi d V_{h}(x)=0,
\end{aligned}
$$


and as a result we obtain

$$
\begin{aligned}
& J_{0}:=\mathbb{E} \int_{M \times \mathbb{R}}\left[\left(\rho_{\varepsilon}^{+}(\omega, t)(x, \xi)\right)^{2}-\rho_{\varepsilon}^{+}(\omega, t)(x, \xi)\right] \theta d \xi d V_{h}(x) \\
& =\mathbb{E} \int_{M \times \mathbb{R}}\left[\left(\rho_{0, \varepsilon}(\omega)(x, \xi)\right)^{2}-\rho_{0, \varepsilon}(\omega)(x, \xi)\right] \theta d \xi d V_{h}(x) \\
& -2 \mathbb{E} \int_{0}^{t} \int_{M \times \mathbb{R}} \theta \rho_{\varepsilon}^{+}(\omega, s)(x, \xi) \operatorname{div}_{h}\left(\rho_{\varepsilon}^{+}(\omega, s)(x, \xi) f_{x}^{\prime}(\xi)\right) d \xi d V_{h}(x) d s \\
& -\mathbb{E} \int_{0}^{t} \int_{M \times \mathbb{R}} \theta \rho_{\varepsilon}^{+}(\omega, s)(x, \xi) \partial_{\xi}\left[\left(G^{2} \nu\right)_{\varepsilon}(\omega, s)(x, \xi)\right] d \xi d V_{h}(x) d s \\
& +2 \mathbb{E} \int_{M \times \mathbb{R}} \int_{0}^{t} \rho_{\varepsilon}^{+}\left(\omega, s_{-}\right)(x, \xi) \theta \partial_{\xi}\left[m_{\varepsilon}(\omega, d s)(x, \xi)\right] d \xi d V_{h}(x) \\
& +2 \mathbb{E} \int_{0}^{t} \int_{M \times \mathbb{R}} \theta \rho_{\varepsilon}^{+}(\omega, s)(x, \xi) A_{\varepsilon}(\omega, s)(x, \xi) d \xi d V_{h}(x) d s \\
& -2 \mathbb{E} \int_{0}^{t} \int_{M \times \mathbb{R}} \theta \rho_{\varepsilon}^{+}(\omega, s)(x, \xi) r_{\varepsilon}(\omega, s)(x, \xi) d \xi d V_{h}(x) d s \\
& +\mathbb{E} \int_{M \times \mathbb{R}} \int_{0}^{t} \sum_{k \geq 1}\left(\left(g_{k} \nu\right)_{\varepsilon}(\omega, s)(x, \xi)\right)^{2} d s \theta d \xi d V_{h}(x) \\
& +\mathbb{E} \int_{M \times \mathbb{R}} \theta \int_{0}^{t} d\left[I_{\varepsilon}(s)(x, \xi)\right] d \xi d V_{h}(x) \\
& +\mathbb{E} \int_{0}^{t} \int_{M \times \mathbb{R}} \theta \operatorname{div}_{h}\left(\rho_{\varepsilon}^{+}(\omega, s)(x, \xi) f_{x}^{\prime}(\xi)\right) d \xi d V_{h}(x) d s \\
& +\frac{1}{2} \mathbb{E} \int_{0}^{t} \int_{M \times \mathbb{R}} \theta \partial_{\xi}\left[\left(G^{2} \nu\right)_{\varepsilon}(\omega, s)(x, \xi)\right] d \xi d V_{h}(x) d s \\
& -\mathbb{E} \int_{M \times \mathbb{R}} \theta \partial_{\xi}\left[m_{\varepsilon}(\omega, t)(x, \xi)\right] d \xi d V_{h}(x) \\
& -\mathbb{E} \int_{0}^{t} \int_{M \times \mathbb{R}} \theta A_{\varepsilon}(\omega, s)(x, \xi) d \xi d V_{h}(x) d s \\
& +\mathbb{E} \int_{0}^{t} \int_{M \times \mathbb{R}} \theta r_{\varepsilon}(\omega, s)(x, \xi) d \xi d V_{h}(x) d s=: J_{1}+\cdots+J_{13},
\end{aligned}
$$

valid for $\varepsilon<\bar{\varepsilon}$ and $0 \leq \theta \in D(M \times \mathbb{R})$.

We are going to send the parameter $\varepsilon$ to 0 . Let us analyze the behavior of the terms in (4.8) separately, starting with the left hand side. By Lemma 4.10, $\rho_{\varepsilon}^{+}(\omega, t)$ converges to $\rho^{+}(\omega, t)$ in $L_{\text {loc }}^{p}(M \times \mathbb{R})$ as $\varepsilon \rightarrow 0$, for all $(\omega, t) \in \Omega_{T}$ and $p \in[1, \infty)$. Moreover, as we have seen in the proof of Lemma 4.8, we can bound $\left|\left(\rho_{\kappa}^{+}\right)_{\varepsilon}(\omega, t)(z, \xi)\right| \leq C(M, h, \mathcal{A}), \forall \kappa \in \mathcal{A}$, uniformly in $\varepsilon,(\omega, t, z, \xi) \in \Omega_{T} \times \mathbb{R}^{n} \times \mathbb{R}$. Therefore, for a different constant,

$$
\left|\rho_{\varepsilon}^{+}(\omega, t)(x, \xi)\right| \leq C(M, h, \mathcal{A}),
$$

for all $(\omega, t, x, \xi) \in \Omega_{T} \times M \times \mathbb{R}$. By the dominated convergence theorem,

$$
\lim _{\varepsilon \rightarrow 0} J_{0}=\mathbb{E} \int_{M \times \mathbb{R}}\left[\rho^{+}(\omega, t, x, \xi)^{2}-\rho^{+}(\omega, t, x, \xi)\right] \theta d \xi d V_{h}(x) .
$$

An analogous result holds for $J_{1}$.

From Lemma 4.10 , we know that $\lim _{\varepsilon \rightarrow 0} r_{\varepsilon}=0$ in $L^{1}\left(\Omega_{T} \times M \times(-L, L)\right)$, for all $L>0$. This property, coupled with $\frac{\varepsilon \rightarrow 0}{4.9}$, enables us to conclude that

$$
J_{13} \stackrel{\varepsilon \downarrow 0}{\longrightarrow} 0, \quad J_{6} \stackrel{\varepsilon \downarrow 0}{\longrightarrow} 0 .
$$


We now deal with the terms $J_{5}$ and $J_{12}$. By Lemma $4.10, \lim _{\varepsilon \rightarrow 0} A_{\varepsilon}(\omega, s)=0$ in $L_{\text {loc }}^{p}(M \times \mathbb{R})$, for all $(\omega, s) \in \Omega_{T}$ and $p \in[1, \infty)$. Furthermore, as in the proof of Lemma 4.6, we see that $\left\|A_{\varepsilon}(\omega, s)\right\|_{L^{1}(\operatorname{supp} \theta)}$ is bounded by an $\varepsilon$-independent constant $C\left(M, h, \mathcal{A}, f^{\prime}, \theta\right)$. The dominated convergence theorem thus implies

$$
J_{12} \stackrel{\varepsilon \downarrow 0}{\longrightarrow} 0 \quad J_{5} \stackrel{\varepsilon \downarrow 0}{\longrightarrow} 0 .
$$

Let us consider the terms involving the $\operatorname{div}_{h}$ operator. Since $\rho_{\varepsilon}^{+}(\omega, s)$ is smooth (in $x$ ) and the $\xi$-derivative of the flux is geometry-compatible $\sqrt{2.1}$, it follows (after an integration by parts) that for any $(\omega, s, \xi) \in \Omega_{T} \times \mathbb{R}$,

$$
\begin{gathered}
-2 \int_{M} \theta \rho_{\varepsilon}^{+}(\omega, s)(x, \xi) \operatorname{div}_{h}\left(\rho_{\varepsilon}^{+}(\omega, s)(x, \xi) f_{x}^{\prime}(\xi)\right) d V_{h}(x) \\
\quad=\int_{M} \rho_{\varepsilon}^{+}(\omega, s)(x, \xi)^{2}\left(f_{x}^{\prime}(\xi), \nabla \theta\right)_{h} d V_{h}(x)
\end{gathered}
$$

Moreover, coupling Lemma 4.10 with $\left|\left(f_{x}^{\prime}(\xi), \nabla \theta\right)\right|_{h} \leq C\left(f^{\prime}, \theta\right)$ on supp $\theta^{\prime}$, we obtain

$$
\begin{aligned}
\int_{M \times \mathbb{R}} & \rho_{\varepsilon}^{+}(\omega, s)(x, \xi)^{2}\left(f_{x}^{\prime}(\xi), \nabla \theta\right)_{h} d \xi d V_{h}(x) \\
& \stackrel{\varepsilon \downarrow 0}{\longrightarrow} \int_{M \times \mathbb{R}} \rho^{+}(\omega, s, x, \xi)^{2}\left(f_{x}^{\prime}(\xi), \nabla \theta\right)_{h} d \xi d V_{h}(x),
\end{aligned}
$$

for all $(\omega, s) \in \Omega_{T}$. Again we can find a bound that is uniform in $\varepsilon$ and $(\omega, s) \in \Omega_{T}$, which permits us to apply the dominated convergence theorem to conclude that

$$
\lim _{\varepsilon} J_{2}=\mathbb{E} \int_{0}^{t} \int_{M \times \mathbb{R}} \rho^{+}(\omega, s, x, \xi)^{2}\left(f_{x}^{\prime}(\xi), \nabla \theta\right)_{h} d \xi d V_{h}(x) d s .
$$

A similar result holds for $J_{9}$.

We now treat the term $J_{11}$. By Lemma 4.10 .

$$
-\int_{M \times \mathbb{R}} \theta \partial_{\xi}\left[m_{\varepsilon}(\omega, t)(x, \xi)\right] d \xi d V_{h}(x) \stackrel{\varepsilon \downarrow 0}{\longrightarrow} \int_{[0, t] \times M \times \mathbb{R}} \partial_{\xi} \theta m(d s, d x, d \xi),
$$

for all $(\omega, t) \in \Omega_{T}$. Furthermore, by Lemma 4.3 summing over $\kappa \in \mathcal{A}$, we obtain

$$
\begin{aligned}
\left|\int_{M \times \mathbb{R}} \theta \partial_{\xi}\left[m_{\varepsilon}(\omega, t)(x, \xi)\right] d \xi d V_{h}(x)\right| & \leq\left\|\partial_{\xi} \theta\right\|_{L^{\infty}(M \times \mathbb{R})} \int_{[0, t] \times M \times \mathbb{R}} m(d s, d x, d \xi) \\
& \leq\left\|\partial_{\xi} \theta\right\|_{L^{\infty}(M \times \mathbb{R})} m(\omega)([0, T] \times M \times \mathbb{R}) .
\end{aligned}
$$

In view of the integrability property $\mathbb{E} m([0, T] \times M \times \mathbb{R})<\infty$, we are allowed to apply the dominated convergence theorem to arrive at

$$
\lim _{\varepsilon \rightarrow 0} J_{11}=\mathbb{E} \int_{[0, t] \times M \times \mathbb{R}} \partial_{\xi} \theta m(d s, d x, d \xi) .
$$

We now deal with the term $J_{10}$. Arguing as above, Lemma 4.10 implies

$$
\begin{aligned}
\int_{M \times \mathbb{R}} & \theta \partial_{\xi}\left[\left(G^{2} \nu\right)_{\varepsilon}(\omega, s)(x, \xi)\right] d \xi d V_{h}(x) \\
& \stackrel{\varepsilon \downarrow 0}{\longrightarrow}-\int_{M}\left(\int_{\mathbb{R}} \partial_{\xi} \theta G^{2}(x, \xi) \nu_{\omega, s, x}(d \xi)\right) d V_{h}(x),
\end{aligned}
$$

for all $(\omega, s) \in \Omega_{T}$. Furthermore, by Lemma 4.5 , summing over $\kappa \in \mathcal{A}$, we obtain

$$
\begin{aligned}
& \mid \int_{M \times \mathbb{R}} \theta \partial_{\xi} {\left[\left(G^{2} \nu\right)_{\varepsilon}(\omega, s)(x, \xi)\right] d \xi d V_{h}(x) \mid } \\
& \leq\left\|\partial_{\xi} \theta\right\|_{L^{\infty}(M \times \mathbb{R})} \int_{M}\left(\int_{\mathbb{R}} G^{2}(x, \xi) \nu_{\omega, s, x}(d \xi)\right) d V_{h}(x),
\end{aligned}
$$


which is integrable over $\Omega \times[0, t]$ by the "vanishing at infinity" assumption, cf. Definition 3.3 . Again by dominated convergence, we conclude

$$
\lim _{\varepsilon \rightarrow 0} J_{10}=-\frac{1}{2} \mathbb{E} \int_{0}^{t} \int_{M}\left(\int_{\mathbb{R}} \partial_{\xi} \theta G^{2}(x, \xi) \nu_{\omega, s, x}(d \xi)\right) d V_{h}(x) d s .
$$

Summarizing, sending $\varepsilon \rightarrow 0$ in 4.8 yields

$$
\begin{aligned}
& \mathbb{E} \int_{M \times \mathbb{R}}\left[\left(\rho^{+}(\omega, t, x, \xi)\right)^{2}-\rho^{+}(\omega, t, x, \xi)\right] \theta d \xi d V_{h}(x) \\
& =\mathbb{E} \int_{M \times \mathbb{R}}\left[\left(\rho_{0}(\omega, x, \xi)\right)^{2}-\rho_{0}(\omega, x, \xi)\right] \theta d \xi d V_{h}(x) \\
& +\mathbb{E} \int_{0}^{t} \int_{M \times \mathbb{R}}\left[\left(\rho^{+}(\omega, s, x, \xi)\right)^{2}-\rho^{+}(\omega, s, x, \xi)\right]\left(f_{x}^{\prime}(\xi), \nabla \theta\right)_{h} d \xi d V_{h}(x) d s \\
& +\limsup _{\varepsilon \rightarrow 0}\left\{\mathbb{E} \int_{0}^{t} \int_{M \times \mathbb{R}} \partial_{\xi} \theta \rho_{\varepsilon}^{+}(\omega, s)(x, \xi)\left(G^{2} \nu\right)_{\varepsilon}(\omega, s)(x, \xi) d \xi d V_{h}(x) d s\right. \\
& -\mathbb{E} \int_{0}^{t} \int_{M \times \mathbb{R}} \theta \nu_{\varepsilon}(\omega, s)(x, \xi)\left(G^{2} \nu\right)_{\varepsilon}(\omega, s)(x, \xi) d \xi d V_{h}(x) d s \\
& +2 \mathbb{E} \int_{M \times \mathbb{R}} \int_{0}^{t} \rho_{\varepsilon}^{+}\left(\omega, s_{-}\right)(x, \xi) \theta \partial_{\xi}\left[m_{\varepsilon}(\omega, d s)(x, \xi)\right] d \xi d V_{h}(x) \\
& +\mathbb{E} \int_{M \times \mathbb{R}} \int_{0}^{t} \sum_{k \geq 1}\left(\left(g_{k} \nu\right)_{\varepsilon}(\omega, s)(x, \xi)\right)^{2} d s \theta d \xi d V_{h}(x) \\
& \left.+\mathbb{E} \int_{M \times \mathbb{R}} \theta \int_{0}^{t} d\left[I_{\varepsilon}(s)(x, \xi)\right] d \xi d V_{h}(x)\right\} \\
& -\frac{1}{2} \mathbb{E} \int_{0}^{t} \int_{M}\left(\int_{\mathbb{R}} \partial_{\xi} \theta G^{2}(x, \xi) \nu_{\omega, x, s}(d \xi)\right) d V_{h}(x) d s \\
& +\mathbb{E} \int_{[0, t] \times M \times \mathbb{R}} \partial_{\xi} \theta m(d s, d x, d \xi),
\end{aligned}
$$

where we have integrated by parts in the $J_{3}$ term and made use of Lemma 4.10 to express the $\xi$-derivative of $\rho_{\varepsilon}^{+}(\omega, s)$ as $-\nu_{\varepsilon}(\omega, s)$.

Before continuing we state three technical lemmas, whose proofs are postponed until the end of the section.

Lemma 4.11. For $\gamma \in C^{0}(M)$ and $\psi \in C_{c}^{1}(\mathbb{R})$,

$$
\begin{aligned}
& \left|\int_{M \times \mathbb{R}} \gamma(x) \partial_{\xi} \psi(\xi) \rho_{\varepsilon}^{+}(\omega, s)(x, \xi)\left(G^{2} \nu\right)_{\varepsilon}(\omega, s)(x, \xi) d \xi d V_{h}(x)\right| \\
& \quad \leq C(M, h, \mathcal{A})\|\gamma\|_{L^{\infty}(M)}\left\|\partial_{\xi} \psi\right\|_{L^{\infty}(\mathbb{R})} \int_{M} \int_{\operatorname{supp} \partial_{\xi} \psi+[-\varepsilon, \varepsilon]} G^{2}(x, \xi) \nu_{\omega, s, x}(d \xi) d V_{h}(x),
\end{aligned}
$$

for all $(\omega, s) \in \Omega_{T}$ and $\varepsilon<\bar{\varepsilon} / 2$.

The next lemma depends crucially on the regularity of the noise coefficients $\left(g_{k}\right)_{k}$, cf. 2.4 and the motivational discussion around (1.6).

Lemma 4.12. Let $0 \leq \psi \in \mathcal{D}(\mathbb{R})$. Then

$$
\begin{aligned}
-\mathbb{E} \int_{0}^{t} & \int_{M \times \mathbb{R}} \psi(\xi) \nu_{\varepsilon}(\omega, s)(x, \xi)\left(G^{2} \nu\right)_{\varepsilon}(\omega, s)(x, \xi) d \xi d V_{h}(x) d s \\
& +\mathbb{E} \int_{M \times \mathbb{R}} \int_{0}^{t} \sum_{k \geq 1}\left(\left(g_{k} \nu\right)_{\varepsilon}(\omega, s)(x, \xi)\right)^{2} d s \psi(\xi) d \xi d V_{h}(x) \stackrel{\varepsilon \downarrow 0}{\longrightarrow} 0 .
\end{aligned}
$$


The last lemma controls a term that involves the measure $m_{\varepsilon}(\omega, d s)(x, \xi)$ for large values of $\xi$.

Lemma 4.13. Let $\psi_{N} \in \mathcal{D}(\mathbb{R}), N \in \mathbb{N}$ with $0 \leq \psi_{N} \leq 1, \psi_{N}(\xi)=1$, if $|\xi| \leq N$, $\psi_{N}(\xi)=0$, if $|\xi| \geq N+1$, and $\left|\partial_{\xi} \psi_{N}(\xi)\right| \leq 2$. Then

$$
\lim _{N \rightarrow \infty} \limsup _{\varepsilon \rightarrow 0}\left|-2 \mathbb{E} \int_{M \times \mathbb{R}} \int_{0}^{t} \partial_{\xi} \psi_{N} \rho_{\varepsilon}^{+}\left(\omega, s_{-}\right)(x, \xi) m_{\varepsilon}(\omega, d s)(x, \xi) d \xi d V_{h}(x)\right|=0 .
$$

Let us continue with the proof of Proposition 4.1. Choose $\theta(x, \xi)=\psi_{N}(\xi)$, where $\psi_{N} \in \mathcal{D}(\mathbb{R}), N \in \mathbb{N}$, and $0 \leq \psi_{N} \leq 1, \psi_{N}(\xi)=1$, if $|\xi| \leq N, \psi_{N}(\xi)=0$, if $|\xi| \geq N+1$, and $\left|\partial_{\xi} \psi_{N}(\xi)\right| \leq 2$.

With this choice of $\theta$, the second term on the right-hand side of 4.10 is zero. Since the kinetic measure $m$ vanishes for large $\xi$ (cf. Definition 3.1),

$$
\begin{aligned}
\left|\mathbb{E} \int_{[0, t] \times M \times \mathbb{R}} \partial_{\xi} \psi_{N} m(d s, d x, d \xi)\right| & \leq 2 \mathbb{E} \int_{[0, t] \times M \times\{\xi: N \leq|\xi| \leq N+1\}} m(d s, d x, d \xi) \\
& \leq 2 \mathbb{E} \int_{[0, T] \times M \times B_{N}^{c}} m(d s, d x, d \xi) \stackrel{N \uparrow \infty}{\longrightarrow} 0 .
\end{aligned}
$$

Moreover, by 2.3,

$$
\begin{aligned}
\mid-\frac{1}{2} \mathbb{E} \int_{0}^{t} \int_{M} & \left(\int_{\mathbb{R}} \partial_{\xi} \psi_{N} G^{2}(x, \xi) \nu_{\omega, s, x}(d \xi)\right) d V_{h}(x) d s \mid \\
\leq & \mathbb{E} \int_{0}^{t} \int_{M}\left(\int_{N \leq|\xi| \leq N+1} D_{1}\left(1+|\xi|^{2}\right) \nu_{\omega, s, x}(d \xi)\right) d V_{h}(x) d s
\end{aligned}
$$

By the "vanishing at infinity" assumption (3.1), applying twice the dominated convergence theorem, we conclude that the above term vanishes as $N \rightarrow \infty$.

Therefore, taking into account Lemma 4.12 as well, the equation 4.10 becomes

$$
\begin{gathered}
\mathbb{E} \int_{M \times \mathbb{R}}\left[\left(\rho^{+}(\omega, t, x, \xi)\right)^{2}-\rho^{+}(\omega, t, x, \xi)\right] \psi_{N} d \xi d V_{h}(x) \\
=\mathbb{E} \int_{M \times \mathbb{R}}\left[\left(\rho_{0}(\omega, x, \xi)\right)^{2}-\rho_{0}(\omega, x, \xi)\right] \psi_{N} d \xi d V_{h}(x) \\
+\limsup _{\varepsilon \rightarrow 0}\left\{\mathbb{E} \int_{0}^{t} \int_{M \times \mathbb{R}} \partial_{\xi} \psi_{N} \rho_{\varepsilon}^{+}(\omega, s)(x, \xi)\left(G^{2} \nu\right)_{\varepsilon}(\omega, s)(x, \xi) d \xi d V_{h}(x) d s\right. \\
+2 \mathbb{E} \int_{M \times \mathbb{R}} \int_{0}^{t} \rho_{\varepsilon}^{+}\left(\omega, s_{-}\right)(x, \xi) \psi_{N} \partial_{\xi}\left[m_{\varepsilon}(\omega, d s)(x, \xi)\right] d \xi d V_{h}(x) \\
\left.+\mathbb{E} \int_{M \times \mathbb{R}} \psi_{N} \int_{0}^{t} d\left[I_{\varepsilon}(s)(x, \xi)\right] d \xi d V_{h}(x)\right\}+o(1),
\end{gathered}
$$

where the error $o(1)$ tends to zero as $N \rightarrow \infty$.

The following integration by parts formula can be verified:

$$
\begin{aligned}
J_{4} & =2 \mathbb{E} \int_{M \times \mathbb{R}} \int_{0}^{t} \rho_{\varepsilon}^{+}\left(\omega, s_{-}\right)(x, \xi) \psi_{N} \partial_{\xi}\left[m_{\varepsilon}(\omega, d s)(x, \xi)\right] d \xi d V_{h}(x) \\
& =-2 \mathbb{E} \int_{M \times \mathbb{R}} \int_{0}^{t} \partial_{\xi}\left(\rho_{\varepsilon}^{+}\left(\omega, s_{-}\right)(x, \xi) \psi_{N}\right) m_{\varepsilon}(\omega, d s)(x, \xi) d \xi d V_{h}(x) .
\end{aligned}
$$

Note that in the deterministic setting one also mollifies in time, in which case 4.12 holds trivially. In the present context the relevant functions are not smooth in the time variable and, as a result, 4.12 is no longer immediate. However, to avoid making this paper even longer, we do not give a detailed proof of 4.12). 
Therefore,

$$
\begin{aligned}
J_{4}= & -2 \mathbb{E} \int_{M \times \mathbb{R}} \int_{0}^{t} \partial_{\xi} \psi_{N} \rho_{\varepsilon}^{+}\left(\omega, s_{-}\right)(x, \xi) m_{\varepsilon}(\omega, d s)(x, \xi) d \xi d V_{h}(x) \\
& +2 \mathbb{E} \int_{M \times \mathbb{R}} \int_{0}^{t} \psi_{N} \nu_{\varepsilon}\left(\omega, s_{-}\right)(x, \xi) m_{\varepsilon}(\omega, d s)(x, \xi) d \xi d V_{h}(x) \\
\geq & -2 \mathbb{E} \int_{M \times \mathbb{R}} \int_{0}^{t} \partial_{\xi} \psi_{N} \rho_{\varepsilon}^{+}\left(\omega, s_{-}\right)(x, \xi) m_{\varepsilon}(\omega, d s)(x, \xi) d \xi d V_{h}(x),
\end{aligned}
$$

because $m_{\varepsilon}(\omega, d s)(x, \xi)$ is a positive $s$-measure (indeed, the function $s \mapsto m_{\varepsilon}(\omega, s)(x, \xi)$ is non-decreasing), and $\psi_{N}, \nu_{\varepsilon}\left(\omega, s_{-}\right)(x, \xi)$ are non-negative functions.

By using Lemma 4.11 with $\gamma \equiv 1$ and $\psi=\psi_{N}$, for $\varepsilon<\bar{\varepsilon} / 4, \varepsilon<1$ and $(\omega, s) \in \Omega_{T}$, we obtain

$$
\begin{aligned}
& \left|\int_{M \times \mathbb{R}} \partial_{\xi} \psi_{N} \rho_{\varepsilon}^{+}(\omega, s)(x, \xi)\left(G^{2} \nu\right)_{\varepsilon}(\omega, s)(x, \xi) d \xi d V_{h}(x)\right| \\
& \quad \leq 2 D_{1} C(M, h, \mathcal{A}) \int_{M} \int_{N-1 \leq|\xi| \leq N+2}\left(1+|\xi|^{2}\right) \nu_{\omega, s, x}(d \xi) d V_{h}(x) .
\end{aligned}
$$

Hence,

$$
\begin{aligned}
\left|\limsup _{\varepsilon \rightarrow 0} \mathbb{E} \int_{0}^{t} \int_{M \times \mathbb{R}} \partial_{\xi} \psi_{N} \rho_{\varepsilon}^{+}(\omega, s)(x, \xi)\left(G^{2} \nu\right)_{\varepsilon}(\omega, s)(x, \xi) d \xi d V_{h}(x) d s\right| \\
\leq \limsup _{\varepsilon}\left|\mathbb{E} \int_{0}^{t} \int_{M \times \mathbb{R}} \partial_{\xi} \psi_{N} \rho_{\varepsilon}^{+}(\omega, s)(x, \xi)\left(G^{2} \nu\right)_{\varepsilon}(\omega, s)(x, \xi) d \xi d V_{h}(x) d s\right| \\
\leq 2 D_{1} C(M, h, \mathcal{A}) \mathbb{E} \int_{0}^{t} \int_{M} \int_{N-1 \leq|\xi| \leq N+2}\left(1+|\xi|^{2}\right) \nu_{\omega, s, x}(d \xi) d V_{h}(x) d s,
\end{aligned}
$$

and once again by the "vanishing at infinity" assumption on $\nu$, cf. (3.1), the last quantity goes to zero as $N \rightarrow \infty$.

Using Lemma 4.13 and the non-negativity of $\int_{0}^{t} d\left[I_{\varepsilon}(s)(x, \xi)\right]$, we arrive at

$$
\begin{aligned}
& \mathbb{E} \int_{M \times \mathbb{R}}\left[\left(\rho^{+}(\omega, t, x, \xi)\right)^{2}-\rho^{+}(\omega, t, x, \xi)\right] \psi_{N} d \xi d V_{h}(x) \\
& \geq \mathbb{E} \int_{M \times \mathbb{R}}\left[\left(\rho_{0}(\omega, x, \xi)\right)^{2}-\rho_{0}(\omega, x, \xi)\right] \psi_{N} d \xi d V_{h}(x)+o(1),
\end{aligned}
$$

where $o(1)$ tends to zero as $N \rightarrow \infty$.

We use the monotone convergence theorem to send $N \rightarrow \infty$, obtaining

$$
\begin{array}{r}
0 \leq \mathbb{E} \int_{M \times \mathbb{R}}\left[\rho^{+}(\omega, t, x, \xi)-\left(\rho^{+}(\omega, t, x, \xi)\right)^{2}\right] d \xi d V_{h}(x) \\
\leq \mathbb{E} \int_{M \times \mathbb{R}}\left[\rho_{0}(\omega, x, \xi)-\left(\rho_{0}(\omega, x, \xi)\right)^{2}\right] d \xi d V_{h}(x),
\end{array}
$$

for all $t \in[0, T]$. This concludes the proof of Proposition 4.1 .

4.5. Uniqueness part of Theorem 3.2. Let us consider a kinetic initial function $\rho_{0}=\mathbb{I}_{u_{0}>\xi}$, with $u_{0} \in L^{\infty}\left(\Omega, \mathcal{F}_{0} ; L^{\infty}(M, h)\right)$. Then the right-hand side of $(4.13)$ is zero. Repeating an argument from [15], there is a function $u^{+}(\omega, t, x)$ such that

$$
\rho^{+}(\omega, t, x, \xi)=\mathbb{I}_{u^{+}(\omega, t, x)>\xi}, \quad \text { for a.e. }(\omega, t, x, \xi) .
$$

The function $u^{+}$constitutes a kinetic solution according to Definition 3.2. A similar result holds for $\rho^{-}$. Since $\rho^{+}(t)=\rho(t)$ for a.e. $t$, it follows that a generalized kinetic solution $\rho$ is in fact a kinetic solution.

Finally, following [54, let us establish the $L^{1}$ contraction principle in Theorem 3.2. Let $u_{1}, u_{2}$ be two kinetic solutions with corresponding initial data $u_{1,0}, u_{2,0}$ and 
kinetic measures $m_{1}, m_{2}$. Set $\rho=\frac{1}{2}\left(\mathbb{I}_{u_{1}>\xi}+\mathbb{I}_{u_{2}>\xi}\right)$ and $\rho_{0}=\frac{1}{2}\left(\mathbb{I}_{u_{1,0}>\xi}+\mathbb{I}_{u_{2,0}>\xi}\right)$. Then $\rho$ is a generalized kinetic solution with initial data $\rho_{0}$, kinetic measure $m=$ $\frac{1}{2}\left(m_{1}+m_{2}\right)$, and $\partial_{\xi} \rho=-\frac{1}{2}\left(\delta_{u_{1}}+\delta_{u_{2}}\right)$. By 4.13 ,

$$
\mathbb{E} \int_{M \times \mathbb{R}}\left(\rho-\rho^{2}\right)(t) d \xi d V_{h}(x) \leq \mathbb{E} \int_{M \times \mathbb{R}}\left(\rho_{0}-\rho_{0}^{2}\right) d \xi d V_{h}(x),
$$

for a.e. $t \in[0, T]$. Using that $\rho_{i}^{2}=\rho_{i}$ for $i=1,2$, a simple computation reveals that $\rho-\rho^{2}=\frac{1}{4}\left(\rho_{1}-\rho_{2}\right)^{2}=\frac{1}{4}\left|\rho_{1}-\rho_{2}\right|$ and so $\int_{\mathbb{R}}\left(\rho-\rho^{2}\right) d \xi=\frac{1}{4}\left|u_{1}-u_{2}\right|$. Similarly, $\int_{\mathbb{R}}\left(\rho_{0}-\rho_{0}^{2}\right) d \xi=\frac{1}{4}\left|u_{1,0}-u_{2,0}\right|$. Therefore, 3.4 holds for a.e. $t \in[0, T]$.

The trajectories of a kinetic solution $u$ are continuous. Indeed, as $u=u^{+}$ a.e. with respect to $(\omega, t, x)$, it is enough to show that $u^{+}$admits $\mathbb{P}$-a.s. continuous trajectories in $L^{p}(M)$. This is achieved by replicating the proof in [15, Cor. 16]. Thanks to the continuity of the trajectories, 3.4 must hold for all $t \in[0, T]$.

\subsection{Proofs of technical lemmas.}

Proof of Lemma 4.11. As usual, we make use of a local argument: fix $\kappa \in \mathcal{A}$ and consider $\gamma \in C_{c}^{0}\left(X_{\kappa}\right)$. Hence, $\gamma \partial_{\xi} \psi \rho_{\varepsilon}^{+}(\omega, s) \in C_{c}^{0}\left(X_{\kappa} \times \mathbb{R}\right)$ and, by means of the coordinates given by $\kappa$,

$$
\begin{aligned}
I: & =\left|\int_{M \times \mathbb{R}} \gamma(x) \partial_{\xi} \psi(\xi) \rho_{\varepsilon}^{+}(\omega, s)(x, \xi) \frac{\left(G^{2} \nu_{\kappa}\right)_{\varepsilon}(\omega, s)(x, \xi)}{\left|h_{\kappa}(x)\right|^{1 / 2}} d \xi d V_{h}(x)\right| \\
= & \left|\int_{\tilde{X}_{\kappa} \times \mathbb{R}} \gamma(z) \partial_{\xi} \psi(\xi) \rho_{\varepsilon}^{+}(\omega, s)(z, \xi)\left(G^{2} \nu_{\kappa}\right)_{\varepsilon}(\omega, s)(z, \xi) d \xi d z\right| \\
= & \left|\left(G^{2} \nu_{\kappa}\right)_{\omega, s}\left(\left(\gamma \partial_{\xi} \psi \rho_{\varepsilon}^{+}(\omega, s)\right)_{\varepsilon}\right)\right| \\
\leq & \left\|\left(\gamma \partial_{\xi} \psi \rho_{\varepsilon}^{+}(\omega, s)\right)_{\varepsilon}\right\|_{L^{\infty}\left(\tilde{X}_{\kappa} \times \mathbb{R}\right)} \\
& \quad \times \int_{S_{\varepsilon}} \alpha_{\kappa}(z) G^{2}(z, \xi)\left|h_{\kappa}(z)\right|^{1 / 2} \nu_{\omega, s, \kappa^{-1}(z)}(d \xi) d z
\end{aligned}
$$

where (as before) $(\cdots)_{\varepsilon}$ means convolution between $\cdots$ and $\phi_{\varepsilon}$, and

$$
S_{\varepsilon}:=\operatorname{supp}\left(\gamma \partial_{\xi} \psi \rho_{\varepsilon}^{+}(\omega, s)\right)_{\varepsilon} \cap\left(\kappa\left(\operatorname{supp} \alpha_{\kappa}\right) \times \mathbb{R}\right) .
$$

Since $\operatorname{supp}\left(\gamma \partial_{\xi} \psi \rho_{\varepsilon}^{+}(\omega, s)\right) \subset \operatorname{supp} \gamma \times \operatorname{supp} \partial_{\xi} \psi$,

$$
S_{\varepsilon} \subset \kappa\left(\operatorname{supp} \alpha_{\kappa}\right) \times\left(\operatorname{supp} \partial_{\xi} \psi+[-\varepsilon, \varepsilon]\right) .
$$

Thus, by basic convolution estimates, we obtain

$$
\begin{aligned}
I \leq \| & \gamma \partial_{\xi} \psi \rho_{\varepsilon}^{+}(\omega, s) \|_{L^{\infty}\left(\tilde{X}_{\kappa} \times \mathbb{R}\right)} \\
& \times \int_{\kappa\left(\operatorname{supp} \alpha_{\kappa}\right)} \alpha_{\kappa}(z) \int_{\operatorname{supp} \partial_{\xi} \psi+[-\varepsilon, \varepsilon]} G^{2}(z, \xi)\left|h_{\kappa}(z)\right|^{1 / 2} \nu_{\omega, s, \kappa^{-1}(z)}(d \xi) d z \\
\leq C & (M, h, \mathcal{A})\|\gamma\|_{L^{\infty}(M)}\left\|\partial_{\xi} \psi\right\|_{L^{\infty}(\mathbb{R})} \\
& \times \int_{M} \alpha_{\kappa}(x) \int_{\operatorname{supp} \partial_{\xi} \psi+[-\varepsilon, \varepsilon]} G^{2}(x, \xi) \nu_{\omega, s, x}(d \xi) d V_{h}(x)
\end{aligned}
$$

which holds for any global $\gamma \in C^{0}(M)$ as well, since the support of $\frac{\left(G^{2} \nu_{\kappa}\right)_{\varepsilon}(\omega, s)}{\left|h_{\kappa}\right|^{1 / 2}}$ is contained in $\kappa^{-1}\left(\mathcal{U}_{\kappa}\right) \times \mathbb{R}$. Summing over $\kappa \in \mathcal{A}$, we obtain the desired result.

Proof of Lemma 4.12. Consider two charts $\kappa, \kappa^{\prime} \in \mathcal{A}$ such that $X_{\kappa} \cap X_{\kappa^{\prime}} \neq \emptyset$ (to avoid trivialities). The coordinates given by $\kappa$ will be denoted $z$ and the ones given 
by $\kappa^{\prime}$ will be called $w$ instead. Our aim is to compute the following quantity for each fixed $k \in \mathbb{N}, \xi \in \mathbb{R}$, and $(\omega, s) \in F$ (cf. discussion before Lemma 4.7):

$$
\begin{aligned}
\mathcal{S}_{k}\left(\kappa, \kappa^{\prime}\right):=\int_{M}(- & \frac{\left(\nu_{\kappa}\right)_{\varepsilon}(\omega, s)(x, \xi)}{\left|h_{\kappa}(x)\right|^{1 / 2}} \frac{\left(g_{k}^{2} \nu_{\kappa^{\prime}}\right)_{\varepsilon}(\omega, s)(x, \xi)}{\left|h_{\kappa^{\prime}}(x)\right|^{1 / 2}} \\
& \left.+\frac{\left(g_{k} \nu_{\kappa}\right)_{\varepsilon}(\omega, s)(x, \xi)}{\left|h_{\kappa}(x)\right|^{1 / 2}} \frac{\left(g_{k} \nu_{\kappa^{\prime}}\right)_{\varepsilon}(\omega, s)(x, \xi)}{\left|h_{\kappa^{\prime}}(x)\right|^{1 / 2}}\right) d V_{h}(x) .
\end{aligned}
$$

To alleviate the notation, we have not made explicit the dependence of $\mathcal{S}_{k}\left(\kappa, \kappa^{\prime}\right)$ on $\xi$ and $(\omega, s)$. To integrate we use the coordinates given by $\kappa$. Being explicit about the definition of the integrand (specifically the role of $\kappa, \kappa^{\prime}$ ), we have

$$
\begin{array}{r}
\mathcal{S}_{k}\left(\kappa, \kappa^{\prime}\right)=\int_{M}\left(-\frac{\left(\nu_{\kappa}\right)_{\varepsilon}(\omega, s)(\kappa(x), \xi)}{\left|h_{\kappa}(\kappa(x))\right|^{1 / 2}} \frac{\left(g_{k}^{2} \nu_{\kappa^{\prime}}\right)_{\varepsilon}(\omega, s)\left(\kappa^{\prime}(x), \xi\right)}{\left|h_{\kappa^{\prime}}\left(\kappa^{\prime}(x)\right)\right|^{1 / 2}}\right. \\
\left.+\frac{\left(g_{k} \nu_{\kappa}\right)_{\varepsilon}(\omega, s)(\kappa(x), \xi)}{\left.h_{\kappa}(\kappa(x))\right|^{1 / 2}} \frac{\left(g_{k} \nu_{\kappa^{\prime}}\right)_{\varepsilon}(\omega, s)\left(\kappa^{\prime}(x), \xi\right)}{\left|h_{\kappa^{\prime}}\left(\kappa^{\prime}(x)\right)\right|^{1 / 2}}\right) d V_{h}(x) \\
=\int_{\kappa\left(X_{\kappa} \cap X_{\kappa^{\prime}}\right)}\left(-\left(\nu_{\kappa}\right)_{\varepsilon}(\omega, s)(z, \xi) \frac{\left(g_{k}^{2} \nu_{\kappa^{\prime}}\right)_{\varepsilon}(\omega, s)\left(\kappa^{\prime} \circ \kappa^{-1}(z), \xi\right)}{\left|h_{\kappa^{\prime}}\left(\kappa^{\prime} \circ \kappa^{-1}(z)\right)\right|^{1 / 2}}\right. \\
+\left(g_{k} \nu_{\kappa}\right)_{\varepsilon}(\omega, s)(z, \xi) \frac{\left(g_{k} \nu_{\kappa^{\prime}}\right)_{\varepsilon}(\omega, s)\left(\kappa^{\prime} \circ \kappa^{-1}(z), \xi\right)}{\left.\left|h_{\kappa^{\prime}}\left(\kappa^{\prime} \circ \kappa^{-1}(z)\right)\right|^{1 / 2}\right) d z} \\
=\int_{\kappa\left(X_{\kappa} \cap X_{\kappa^{\prime}}\right)} \frac{\left|\operatorname{Jac}\left(\kappa^{\prime} \circ \kappa^{-1}\right)(z)\right|}{\left|h_{\kappa}(z)\right|^{1 / 2}} \\
\quad \times\left(\left(-\left(\nu_{\kappa}\right)_{\varepsilon}(\omega, s)(z, \xi)\left(g_{k}^{2} \nu_{\kappa^{\prime}}\right)_{\varepsilon}(\omega, s)\left(\kappa^{\prime} \circ \kappa^{-1}(z), \xi\right)\right.\right. \\
\left.\quad+\left(g_{k} \nu_{\kappa}\right)_{\varepsilon}(\omega, s)(z, \xi)\left(g_{k} \nu_{\kappa^{\prime}}\right)_{\varepsilon}(\omega, s)\left(\kappa^{\prime} \circ \kappa^{-1}(z), \xi\right)\right) d z .
\end{array}
$$

Note the attendance of $\kappa, \kappa^{\prime}$. Indeed, recall that our functions are defined on the Euclidean space and then lifted to $M$ via the charts $\kappa$ and $\kappa^{\prime}$.

For convenience set $\Phi:=\kappa^{\prime} \circ \kappa^{-1}$. By definition of the involved quantities,

$$
\begin{aligned}
& -\left(\nu_{\kappa}\right)_{\varepsilon}(\omega, s)(z, \xi)\left(g_{k}^{2} \nu_{\kappa^{\prime}}\right)_{\varepsilon}(\omega, s)\left(\kappa^{\prime} \circ \kappa^{-1}(z), \xi\right) \\
& \left.+\left(g_{k} \nu_{\kappa}\right)_{\varepsilon}(\omega, s)(z, \xi)\left(g_{k} \nu_{\kappa^{\prime}}\right)_{\varepsilon}(\omega, s)\left(\kappa^{\prime} \circ \kappa^{-1}(z), \xi\right)\right) \\
& =-\int_{\kappa\left(\operatorname{supp} \alpha_{\kappa}\right) \times \mathbb{R}} \alpha_{\kappa}(\bar{z})\left|h_{\kappa}(\bar{z})\right|^{1 / 2} \phi_{\varepsilon}(z-\bar{z}, \xi-\bar{\xi}) \nu_{\omega, s, \kappa^{-1}(\bar{z})}(d \bar{\xi}) d \bar{z} \\
& \quad \times \int_{\kappa^{\prime}\left(\operatorname{supp} \alpha_{\kappa^{\prime}}\right) \times \mathbb{R}} \alpha_{\kappa^{\prime}}(\bar{w})\left|h_{\kappa^{\prime}}(\bar{w})\right|^{1 / 2} g_{k}^{2}(\bar{w}, \overline{\bar{\xi}}) \\
& \quad \times \phi_{\varepsilon}(\Phi(z)-\bar{w}, \xi-\overline{\bar{\xi}}) \nu_{\omega, s, \kappa^{\prime-1}(\bar{w})}(d \overline{\bar{\xi}}) d \bar{w} \\
& +\int_{\kappa\left(\operatorname{supp} \alpha_{\kappa}\right) \times \mathbb{R}} \alpha_{\kappa}(\bar{z})\left|h_{\kappa}(\bar{z})\right|^{1 / 2} g_{k}(\bar{z}, \bar{\xi}) \phi_{\varepsilon}(z-\bar{z}, \xi-\bar{\xi}) \nu_{\omega, s, \kappa^{-1}(\bar{z})}(d \bar{\xi}) d \bar{z} \\
& \quad \times \int_{\kappa^{\prime}\left(\operatorname{supp} \alpha_{\kappa^{\prime}}\right) \times \mathbb{R}} \alpha_{\kappa^{\prime}}(\bar{w})\left|h_{\kappa^{\prime}}(\bar{w})\right|^{1 / 2} g_{k}(\bar{w}, \overline{\bar{\xi}}) \\
& \times \phi_{\left(\kappa\left(\operatorname{supp} \alpha_{\kappa}\right) \times \mathbb{R}\right) \times\left(\kappa^{\prime}\left(\operatorname{supp} \alpha_{\kappa^{\prime}}\right) \times \mathbb{R}\right)}\left[-g_{k}^{2}(\bar{w}, \overline{\bar{\xi}})+g_{k}(\bar{z}, \bar{\xi}) g_{k}(\bar{w}, \overline{\bar{\xi}})\right] \\
& \quad \times \phi_{\varepsilon}(z-\bar{z}, \xi-\bar{\xi}) \phi_{\varepsilon}(\Phi(z)-\overline{\bar{\xi}}, \xi-\overline{\bar{\xi}})\left(\nu_{\kappa}\right)_{\omega, s} \otimes\left(\nu_{\kappa^{\prime}}\right)_{\omega, s}(d \bar{z}, d \bar{\xi}, d \bar{w}, d \overline{\bar{\xi}}) .
\end{aligned}
$$


Summarizing our findings so far,

$$
\begin{gathered}
\mathcal{S}_{k}\left(\kappa, \kappa^{\prime}\right)=\int_{\kappa\left(X_{\kappa} \cap X_{\kappa^{\prime}}\right)} \frac{|\operatorname{Jac}(\Phi)(z)|}{\left|h_{\kappa}(z)\right|^{1 / 2}} \\
\times\left(\int_{\left(\kappa\left(\operatorname{supp} \alpha_{\kappa}\right) \times \mathbb{R}\right) \times\left(\kappa^{\prime}\left(\operatorname{supp} \alpha_{\kappa^{\prime}}\right) \times \mathbb{R}\right)}\left[-g_{k}^{2}(\bar{w}, \overline{\bar{\xi}})+g_{k}(\bar{z}, \bar{\xi}) g_{k}(\bar{w}, \overline{\bar{\xi}})\right]\right. \\
\times \phi_{\varepsilon}(z-\bar{z}, \xi-\bar{\xi}) \phi_{\varepsilon}(\Phi(z)-\bar{w}, \xi-\overline{\bar{\xi}}) \\
\left.\times\left(\nu_{\kappa}\right)_{\omega, s} \otimes\left(\nu_{\kappa^{\prime}}\right)_{\omega, s}(d \bar{z}, d \bar{\xi}, d \bar{w}, d \overline{\bar{\xi}})\right) d z
\end{gathered}
$$

with $\kappa, \kappa^{\prime} \in \mathcal{A}$ such that $X_{\kappa} \cap X_{\kappa^{\prime}} \neq \emptyset$.

Reversing the roles of $\kappa$ and $\kappa^{\prime}$, we make the following crucial observation:

$$
\begin{aligned}
& \mathcal{S}_{k}\left(\kappa^{\prime}, \kappa\right)=\int_{\kappa\left(X_{\kappa} \cap X_{\kappa^{\prime}}\right)} \frac{|\operatorname{Jac}(\Phi)(z)|}{\left|h_{\kappa}(z)\right|^{1 / 2}} \\
& \times\left(\int_{\left(\kappa\left(\operatorname{supp} \alpha_{\kappa}\right) \times \mathbb{R}\right) \times\left(\kappa^{\prime}\left(\operatorname{supp} \alpha_{\kappa^{\prime}}\right) \times \mathbb{R}\right)}\left[-g_{k}^{2}(\bar{z}, \bar{\xi})+g_{k}(\bar{z}, \bar{\xi}) g_{k}(\bar{w}, \overline{\bar{\xi}})\right]\right. \\
& \times \phi_{\varepsilon}(z-\bar{z}, \xi-\bar{\xi}) \phi_{\varepsilon}(\Phi(z)-\bar{w}, \xi-\overline{\bar{\xi}}) \\
&\left.\quad \times\left(\nu_{\kappa}\right)_{\omega, s} \otimes\left(\nu_{\kappa^{\prime}}\right)_{\omega, s}(d \bar{z}, d \bar{\xi}, d \bar{w}, d \overline{\bar{\xi}})\right) d z
\end{aligned}
$$

where we are again using the coordinates $z$ given by $\kappa$ to integrate.

Therefore, we arrive at the following remarkable identity:

$$
\begin{aligned}
& \mathcal{S}_{k}\left(\kappa, \kappa^{\prime}\right)+\mathcal{S}_{k}\left(\kappa^{\prime}, \kappa\right)=\int_{\kappa\left(X_{\kappa} \cap X_{\kappa^{\prime}}\right)} \frac{|\operatorname{Jac}(\Phi)(z)|}{\left|h_{\kappa}(z)\right|^{1 / 2}} \\
& \times\left(\int_{\left(\kappa\left(\operatorname{supp} \alpha_{\kappa}\right) \times \mathbb{R}\right) \times\left(\kappa^{\prime}\left(\operatorname{supp} \alpha_{\kappa^{\prime}}\right) \times \mathbb{R}\right)}-\left|g_{k}(\bar{z}, \bar{\xi})-g_{k}(\bar{w}, \overline{\bar{\xi}})\right|^{2}\right. \\
& \times \phi_{\varepsilon}(z-\bar{z}, \xi-\bar{\xi}) \phi_{\varepsilon}(\Phi(z)-\bar{w}, \xi-\overline{\bar{\xi}}) \\
&\left.\times\left(\nu_{\kappa}\right)_{\omega, s} \otimes\left(\nu_{\kappa^{\prime}}\right)_{\omega, s}(d \bar{z}, d \bar{\xi}, d \bar{w}, d \overline{\bar{\xi}})\right) d z .
\end{aligned}
$$

In view of the previous computations, we can write (for $k \in \mathbb{N}$ )

$$
\begin{aligned}
& \mathcal{C}_{k}(\varepsilon):=\int_{M}\left(-\nu_{\varepsilon}(\omega, s)(x, \xi)\left(g_{k}^{2} \nu\right)_{\varepsilon}(\omega, s)(x, \xi)+\left(\left(g_{k} \nu\right)_{\varepsilon}(\omega, s)(x, \xi)\right)^{2}\right) d V_{h}(x) \\
& =\sum_{\substack{\kappa, \kappa^{\prime} \in \mathcal{A} \\
X_{\kappa} \cap X_{\kappa^{\prime}} \neq \emptyset}} \mathcal{S}_{k}\left(\kappa, \kappa^{\prime}\right)=\frac{1}{2} \sum_{\substack{\kappa, \kappa^{\prime} \in \mathcal{A} \\
X_{\kappa} \cap X_{\kappa^{\prime}} \neq \emptyset}} \mathcal{S}_{k}\left(\kappa, \kappa^{\prime}\right)+\frac{1}{2} \sum_{\substack{\kappa, \kappa^{\prime} \in \mathcal{A} \\
X_{\kappa} \cap X_{\kappa^{\prime}} \neq \emptyset}} \mathcal{S}_{k}\left(\kappa^{\prime}, \kappa\right) \\
& =-\frac{1}{2} \sum_{\substack{\kappa, \kappa^{\prime} \in \mathcal{A} \\
X_{\kappa} \cap X_{\kappa^{\prime}} \neq \emptyset}} \int_{\kappa\left(X_{\kappa} \cap X_{\kappa^{\prime}}\right)} \frac{|\operatorname{Jac}(\Phi)(z)|}{\left|h_{\kappa}(z)\right|^{1 / 2}} \\
& \times\left(\int_{\left(\kappa\left(\operatorname{supp} \alpha_{\kappa}\right) \times \mathbb{R}\right) \times\left(\kappa^{\prime}\left(\operatorname{supp} \alpha_{\kappa^{\prime}}\right) \times \mathbb{R}\right)}\left|g_{k}(\bar{z}, \bar{\xi})-g_{k}(\bar{w}, \overline{\bar{\xi}})\right|^{2}\right. \\
& \times \phi_{\varepsilon}(z-\bar{z}, \xi-\bar{\xi}) \phi_{\varepsilon}(\Phi(z)-\bar{w}, \xi-\overline{\bar{\xi}}) \\
& \left.\times\left(\nu_{\kappa}\right)_{\omega, s} \otimes\left(\nu_{\kappa^{\prime}}\right)_{\omega, s}(d \bar{z}, d \bar{\xi}, d \bar{w}, d \overline{\bar{\xi}})\right) d z,
\end{aligned}
$$

where we have suppressed the dependency on $\omega, s, \xi$ in $\mathcal{C}_{k}(\varepsilon)$. Note $\mathcal{C}_{k}(\varepsilon) \leq 0$. 
Let us assume for the moment that each $g_{k} \in C^{0}(\mathbb{R})$ is independent of $x \in M$. Then, summing over $k \in \mathbb{N}$, using (2.4) and Lemma 4.10, we obtain

$$
\begin{aligned}
& \mathcal{C}(\varepsilon):=\int_{M}\left(-\nu_{\varepsilon}(\omega, s)(x, \xi)\left(G^{2} \nu\right)_{\varepsilon}(\omega, s)(x, \xi)+\sum_{k \geq 1}\left(\left(g_{k} \nu\right)_{\varepsilon}(\omega, s)(x, \xi)\right)^{2}\right) d V_{h}(x) \\
& =\sum_{k \geq 1} \mathcal{C}_{k}(\varepsilon) \\
& \geq-\frac{D_{2}}{2} \sum_{\substack{\kappa, \kappa^{\prime} \in \mathcal{A} \\
X_{\kappa} \cap X_{\kappa^{\prime}} \neq \emptyset}} \int_{\kappa\left(X_{\kappa} \cap X_{\kappa^{\prime}}\right)} \frac{|\operatorname{Jac}(\Phi)(z)|}{\left|h_{\kappa}(z)\right|^{1 / 2}} \\
& \times\left(\int_{\left(\kappa\left(\operatorname{supp} \alpha_{\kappa}\right) \times \mathbb{R}\right) \times\left(\kappa^{\prime}\left(\operatorname{supp} \alpha_{\kappa^{\prime}}\right) \times \mathbb{R}\right)}|\bar{\xi}-\overline{\bar{\xi}}|^{2} \phi_{\varepsilon}(z-\bar{z}, \xi-\bar{\xi})\right. \\
& \left.\times \phi_{\varepsilon}(\Phi(z)-\bar{w}, \xi-\overline{\bar{\xi}})\left(\nu_{\kappa}\right)_{\omega, s} \otimes\left(\nu_{\kappa^{\prime}}\right)_{\omega, s}(d \bar{z}, d \bar{\xi}, d \bar{w}, d \overline{\bar{\xi}})\right) d z \\
& =-\frac{D_{2}}{2} \sum_{\substack{\kappa, \kappa^{\prime} \in \mathcal{A} \\
X_{\kappa} \cap X_{\kappa^{\prime}} \neq \emptyset}} \int_{\kappa\left(X_{\kappa} \cap X_{\kappa^{\prime}}\right)} \frac{|\operatorname{Jac}(\Phi)(z)|}{\left|h_{\kappa}(z)\right|^{1 / 2}} \\
& \times \int_{\kappa\left(\operatorname{supp} \alpha_{\kappa}\right) \times \kappa^{\prime}\left(\operatorname{supp} \alpha_{\kappa^{\prime}}\right)} \alpha_{\kappa}(\bar{z}) \alpha_{\kappa^{\prime}}(\bar{w})\left|h_{\kappa}(\bar{z})\right|^{1 / 2}\left|h_{\kappa^{\prime}}(\bar{w})\right|^{1 / 2} \\
& \times \varepsilon^{-n} \phi_{1}\left(\frac{z-\bar{z}}{\varepsilon}\right) \varepsilon^{-n} \phi_{1}\left(\frac{\Phi(z)-\bar{w}}{\varepsilon}\right) \Lambda_{\varepsilon, \kappa, \kappa^{\prime}}(\omega, s, \bar{z}, \bar{w}, \xi) d \bar{z} d \bar{w} d z,
\end{aligned}
$$

where

$$
\begin{aligned}
& \Lambda_{\varepsilon, \kappa, \kappa^{\prime}}(\omega, s, \bar{z}, \bar{w}, \xi) \\
& \quad:=\int_{\mathbb{R}^{2}}|\bar{\xi}-\overline{\bar{\xi}}|^{2} \varepsilon^{-1} \phi_{2}\left(\frac{\xi-\bar{\xi}}{\varepsilon}\right) \varepsilon^{-1} \phi_{2}\left(\frac{\xi-\overline{\bar{\xi}}}{\varepsilon}\right) \nu_{\omega, s, \kappa^{-1}(\bar{z})} \otimes \nu_{\omega, s, \kappa^{\prime-1}(\bar{w})}(d \bar{\xi}, d \overline{\bar{\xi}}) .
\end{aligned}
$$

Let $\psi \in \mathcal{D}(\mathbb{R}), \psi \geq 0$. In view of Lemma 8.2 (in the appendix),

$$
0 \leq \int_{\mathbb{R}} \psi(\xi) \Lambda_{\varepsilon, \kappa, \kappa^{\prime}}(\omega, s, \bar{z}, \bar{w}, \xi) d \xi \leq 4\|\psi\|_{L^{\infty}}\left\|\phi_{2}\right\|_{L^{\infty}} \varepsilon
$$

uniformly in $\omega, s, \bar{z}, \bar{w}, \kappa, \kappa^{\prime}$.

Hence, integrating (4.14 with respect to $\psi d \xi$ leads to

$$
\begin{array}{r}
0 \geq \int_{\mathbb{R}} \psi(\xi) \mathcal{C}(\varepsilon) d \xi \geq-\varepsilon C_{\psi} \sum_{\substack{\kappa, \kappa^{\prime} \in \mathcal{A} \\
X_{\kappa} \cap X_{\kappa^{\prime}} \neq \emptyset}} \int_{\kappa\left(X_{\kappa} \cap X_{\kappa^{\prime}}\right)} \frac{|\operatorname{Jac}(\Phi)(z)|}{\left|h_{\kappa}(z)\right|^{1 / 2}} \\
\quad \times \int_{\kappa\left(\operatorname{supp} \alpha_{\kappa}\right) \times \kappa^{\prime}\left(\operatorname{supp} \alpha_{\kappa^{\prime}}\right)} \alpha_{\kappa}(\bar{z}) \alpha_{\kappa^{\prime}}(\bar{w})\left|h_{\kappa}(\bar{z})\right|^{1 / 2}\left|h_{\kappa^{\prime}}(\bar{w})\right|^{1 / 2} \\
\times \varepsilon^{-n} \phi_{1}\left(\frac{z-\bar{z}}{\varepsilon}\right) \varepsilon^{-n} \phi_{1}\left(\frac{\Phi(z)-\bar{w}}{\varepsilon}\right) d \bar{z} d \bar{w} d z \\
\geq-\varepsilon C_{\psi} C_{M, h, \mathcal{A}} \sum_{\substack{\kappa, \kappa^{\prime} \in \mathcal{A} \\
X_{\kappa} \cap X_{\kappa^{\prime}} \neq \emptyset}} \int_{\kappa\left(X_{\kappa} \cap X_{\kappa^{\prime}}\right)} \frac{|\operatorname{Jac}(\Phi)(z)|}{\left|h_{\kappa}(z)\right|^{1 / 2}} d z \geq-\varepsilon C^{\prime},
\end{array}
$$


for some ( $\varepsilon$-independent) constants $C_{\psi}, C_{M, h, \mathcal{A}}, C^{\prime} \geq 0$. This inequality holds for a.e. $(\omega, s) \in \Omega_{T}$. Integrating 4.15 over $\Omega \times[0, t]$, we obtain

$$
0 \geq \mathbb{E} \int_{0}^{t} \int_{\mathbb{R}} \psi(\xi) \mathcal{C}(\varepsilon) d \xi \geq-\varepsilon C^{\prime} t
$$

concluding the proof of Lemma 4.12 in the case where each $g_{k}$ is independent of $x$.

Let us consider the general ( $x$-dependent case). First of all, we may assume that for any $\kappa \in \mathcal{A}$ there exists $C_{\kappa}>1$ such that $h_{\kappa}(x) \leq C_{\kappa}$ Id in the sense of bilinear forms for all $x \in X_{\kappa}$, where Id is the identity matrix on $\mathbb{R}^{n}$. Indeed, if it is not the case, we can choose for any $x \in M$ normal coordinates centered at $x$, and by using the fact the $M$ is compact, we obtain a finite atlas $\mathcal{A}$ for which the above property is fulfilled, and furthermore $\tilde{X}_{\kappa}$ is convex. It follows that

$$
d_{h}(x, y) \leq C_{\kappa}|\kappa(x)-\kappa(y)|, \quad x, y \in X_{\kappa},
$$

where $|\cdot|$ is the Euclidean distance on $\mathbb{R}^{n}$. As a result, 2.4 takes the form

$$
\sum_{k \geq 1}\left|g_{k}(\bar{z}, \bar{\xi})-g_{k}(\bar{w}, \overline{\bar{\xi}})\right|^{2} \leq D_{2}\left(C_{\kappa}\left|\bar{z}-\Phi^{-1}(\bar{w})\right|^{2}+|\bar{\xi}-\overline{\bar{\xi}}|^{2}\right)
$$

for all $\bar{z} \in X_{\kappa}, \bar{w} \in \kappa^{\prime}\left(X_{\kappa} \cap X_{\kappa^{\prime}}\right)$, and $\bar{\xi}, \overline{\bar{\xi}} \in \mathbb{R}$.

By direct inspection of the expression for $\mathcal{S}_{k}\left(\kappa, \kappa^{\prime}\right)$, the integral is actually zero if $\kappa^{-1}\left(\mathcal{U}_{\kappa}\right) \cap \kappa^{\prime-1}\left(\mathcal{U}_{\kappa^{\prime}}\right)=\emptyset$. Therefore, the expression must be studied only for $z \in \mathcal{U}_{\kappa} \cap \Phi^{-1}\left(\mathcal{U}_{\kappa^{\prime}}\right), \bar{z} \in B_{\varepsilon}(z)$, and $\bar{w} \in B_{\varepsilon}(\Phi(z))$. By the construction of $\mathcal{U}_{\kappa}$ and the choice of $\varepsilon, B_{\varepsilon}(z) \subset \tilde{X}_{\kappa}$ and $B_{\varepsilon}(\Phi(z)) \subset \kappa^{\prime}\left(X_{\kappa} \cap X_{\kappa^{\prime}}\right)$. Hence, for all such points, 4.16 clearly holds.

Restarting from (4.14), we now obtain

$$
\begin{aligned}
& \mathcal{C}(\varepsilon) \geq \mathcal{I}(\varepsilon)-\frac{D_{2}}{2} \max _{\kappa} C_{\kappa} \sum_{\substack{\kappa, \kappa^{\prime} \in \mathcal{A} \\
X_{\kappa} \cap X_{\kappa^{\prime}} \neq \emptyset}} \int_{\mathcal{U}_{\kappa} \cap \Phi^{-1}\left(\mathcal{U}_{\kappa^{\prime}}\right)} \frac{|\operatorname{Jac}(\Phi)(z)|}{\left|h_{\kappa}(z)\right|^{1 / 2}} \\
& \times\left(\int_{\left(B_{\varepsilon}(z) \times \mathbb{R}\right) \times\left(B_{\varepsilon}(\Phi(z)) \times \mathbb{R}\right)}\left|\bar{z}-\Phi^{-1}(\bar{w})\right|^{2} \phi_{\varepsilon}(z-\bar{z}, \xi-\bar{\xi})\right. \\
& \left.\times \phi_{\varepsilon}(\Phi(z)-\bar{w}, \xi-\overline{\bar{\xi}})\left(\nu_{\kappa}\right)_{\omega, s} \otimes\left(\nu_{\kappa^{\prime}}\right)_{\omega, s}(d \bar{z}, d \bar{\xi}, d \bar{w}, d \overline{\bar{\xi}})\right) d z \\
& =\mathcal{I}(\varepsilon)-\frac{D_{2}}{2} \max _{\kappa} C_{\kappa} \sum_{\substack{\kappa, \kappa^{\prime} \in \mathcal{A} \\
X_{\kappa} \cap X_{\kappa^{\prime}} \neq \emptyset}} \int_{\mathcal{U}_{\kappa} \cap \Phi^{-1}\left(\mathcal{U}_{\kappa^{\prime}}\right)} \frac{|\operatorname{Jac}(\Phi)(z)|}{\left|h_{\kappa}(z)\right|^{1 / 2}} \\
& \times \int_{B_{\varepsilon}(z) \times B_{\varepsilon}(\Phi(z))}\left|\bar{z}-\Phi^{-1}(\bar{w})\right|^{2} \alpha_{\kappa}(\bar{z}) \alpha_{\kappa^{\prime}}(\bar{w})\left|h_{\kappa}(\bar{z})\right|^{1 / 2}\left|h_{\kappa^{\prime}}(\bar{w})\right|^{1 / 2} \\
& \times \varepsilon^{-n} \phi_{1}\left(\frac{z-\bar{z}}{\varepsilon}\right) \varepsilon^{-n} \phi_{1}\left(\frac{\Phi(z)-\bar{w}}{\varepsilon}\right) \bar{\Lambda}_{\varepsilon, \kappa, \kappa^{\prime}}(\omega, s, \bar{z}, \bar{w}, \xi) d \bar{z} d \bar{w} d z,
\end{aligned}
$$

where $\mathcal{I}(\varepsilon)$ denotes the corresponding term in the $x$-independent case, and

$$
\begin{aligned}
& \bar{\Lambda}_{\varepsilon, \kappa, \kappa^{\prime}}(\omega, s, \bar{z}, \bar{w}, \xi) \\
& \quad:=\int_{\mathbb{R}^{2}} \varepsilon^{-1} \phi_{2}\left(\frac{\xi-\bar{\xi}}{\varepsilon}\right) \varepsilon^{-1} \phi_{2}\left(\frac{\xi-\overline{\bar{\xi}}}{\varepsilon}\right) \nu_{\omega, s, \kappa^{-1}(\bar{z})} \otimes \nu_{\omega, s, \kappa^{\prime-1}(\bar{w})}(d \bar{\xi}, d \overline{\bar{\xi}}) .
\end{aligned}
$$

Let $0 \leq \psi \in \mathcal{D}(\mathbb{R})$. Arguing as in the proof of Lemma 8.2 .

$$
0 \leq \int_{\mathbb{R}} \psi(\xi) \bar{\Lambda}_{\varepsilon, \kappa, \kappa^{\prime}}(\omega, s, \bar{z}, \bar{w}, \xi) d \xi \leq\|\psi\|_{L^{\infty}}\left\|\phi_{2}\right\|_{L^{\infty}} \varepsilon^{-1},
$$


uniformly in $\omega, s, \bar{z}, \bar{w}, \kappa, \kappa^{\prime}$. There is a constant $C_{\Phi}$ such that $\left|\bar{z}-\Phi^{-1}(\bar{w})\right| \leq$ $\left(C_{\Phi}+1\right) \varepsilon$, for all $\bar{z} \in B_{\varepsilon}(z)$ and $\bar{w} \in B_{\varepsilon}(\Phi(z))$. Hence, integrating the above lower bound on $\mathcal{C}(\varepsilon)$ against $\psi d \xi$ we obtain

$$
\begin{aligned}
0 & \geq \int_{\mathbb{R}} \psi(\xi) \mathcal{C}(\varepsilon) d \xi \\
& \geq-\varepsilon C^{\prime}-C_{\psi} \frac{D_{2}}{2} \max _{\kappa} C_{\kappa} \sum_{\substack{\kappa, \kappa^{\prime} \in \mathcal{A} \\
X_{\kappa} \cap X_{\kappa^{\prime}} \neq \emptyset}} \int_{\mathcal{U}_{\kappa} \cap \Phi^{-1}\left(\mathcal{U}_{\kappa^{\prime}}\right)} \frac{|\operatorname{Jac}(\Phi)(z)|}{\left|h_{\kappa}(z)\right|^{1 / 2}} C_{M, h, \mathcal{A}} \varepsilon^{2} \varepsilon^{-1} d z \\
& \geq-\varepsilon C^{\prime}-\varepsilon C^{\prime \prime},
\end{aligned}
$$

for some constants $C_{M, h, \mathcal{A}}, C^{\prime \prime}$, whereas $C^{\prime}$ derives from 4.15. Integrating over $\Omega \times[0, t]$, we conclude as in the $x$-independent case.

Proof of Lemma 4.13. Set $J_{N}:=\{\xi \in \mathbb{R}: N \leq|\xi| \leq N+1\}$. We have

$$
\begin{aligned}
\mathcal{I}(\varepsilon):= & -2 \mathbb{E} \int_{M \times \mathbb{R}} \int_{0}^{t} \partial_{\xi} \psi_{N} \rho_{\varepsilon}^{+}\left(\omega, s_{-}\right)(x, \xi) m_{\varepsilon}(\omega, d s)(x, \xi) d \xi d V_{h}(x) \mid \\
& \leq 4 C(M, h, \mathcal{A}) \mathbb{E} \int_{M \times J_{N}} \int_{0}^{t}\left|m_{\varepsilon}(\omega, d s)(x, \xi)\right| d \xi d V_{h}(x),
\end{aligned}
$$

where $\left|m_{\varepsilon}(\omega, d s)(x, \xi)\right|$ is the total variation of the $s$-measure $m_{\varepsilon}(\omega, d s)(x, \xi)$. Since $s \mapsto m_{\varepsilon}(\omega, s)(x, \xi)$ is non-decreasing, the expression on the right-hand side equals

$$
4 C(M, h, \mathcal{A}) \mathbb{E} \int_{M \times J_{N}} m_{\varepsilon}(\omega, t)(x, \xi) d \xi d V_{h}(x) .
$$

Since $M \times J_{N}$ is compact, weak convergence of measures implies that

$$
\limsup _{\varepsilon \rightarrow 0} \int_{M \times J_{N}} m_{\varepsilon}(\omega, t)(x, \xi) d \xi d V_{h}(x) \leq \int_{[0, t] \times M \times J_{N}} m(d s, d x, d \xi),
$$

for any $(\omega, t) \in \Omega_{T}$ and $N \in \mathbb{N}$. Suppose that there exists a dominant integrable $H=H(\omega)$ such that

$$
\int_{M \times J_{N}} m_{\varepsilon}(\omega, t)(x, \xi) d \xi d V_{h}(x) \leq H(\omega)
$$

uniformly in $\varepsilon$. Then, by the reverse Fatou lemma, we infer

$$
\begin{aligned}
\limsup _{\varepsilon \rightarrow 0} \mathcal{I}(\varepsilon) & \leq 4 C(M, h, \mathcal{A}) \underset{\varepsilon \rightarrow 0}{\limsup } \mathbb{E} \int_{M \times J_{N}} m_{\varepsilon}(\omega, t)(x, \xi) d \xi d V_{h}(x) \\
& \leq 4 C(M, h, \mathcal{A}) \mathbb{E} \limsup _{\varepsilon \rightarrow 0} \int_{M \times J_{N}} m_{\varepsilon}(\omega, t)(x, \xi) d \xi d V_{h}(x) \\
& \leq 4 C(M, h, \mathcal{A}) \mathbb{E} \int_{[0, t] \times M \times J_{N}} m(d s, d x, d \xi),
\end{aligned}
$$

valid for any $t \in[0, T]$ and $N \in \mathbb{N}$. Since the kinetic measure $m$ vanishes for large $\xi$, the last quantity converges to zero as $N \rightarrow \infty$.

We are hence left to find a suitable $H$. We argue like this: let $\kappa \in \mathcal{A},(\omega, t) \in \Omega_{T}$, and $\varepsilon<\bar{\varepsilon}$. For $\psi \in C_{c}^{0}\left(\mathbb{R}^{n} \times \mathbb{R}\right), 0 \leq \psi \leq 1$, we compute (functions are identified with measures)

$$
\begin{aligned}
\left(\left(\alpha_{\kappa} m\right)_{\sharp}\right)_{\varepsilon}(\omega, t)(\psi) & =\int_{\mathbb{R}^{n} \times \mathbb{R}} \psi_{\varepsilon}(z, \xi)\left(\alpha_{\kappa} m\right)_{\sharp}(\omega, t)(d z, d \xi) \\
& \leq\left\|\psi_{\varepsilon}\right\|_{L^{\infty}}\left(\alpha_{\kappa} m\right)_{\sharp}(\omega, t)\left(\mathbb{R}^{n} \times \mathbb{R}\right) \\
& \leq\|\psi\|_{L^{\infty}}\left(\alpha_{\kappa} m\right)_{\sharp}(\omega, t)\left(\mathbb{R}^{n} \times \mathbb{R}\right) \\
& \leq\left(\alpha_{\kappa} m\right)_{\sharp}(\omega, t)\left(\mathbb{R}^{n} \times \mathbb{R}\right),
\end{aligned}
$$


where, as usual, $(\cdots)_{\varepsilon}$ means convolution between $\cdots$ and $\phi_{\varepsilon}$. Thus, taking the supremum over such $\psi$, we infer

$$
\left(\left(\alpha_{\kappa} m\right)_{\sharp}\right)_{\varepsilon}(\omega, t)\left(\mathbb{R}^{n} \times \mathbb{R}\right) \leq\left(\alpha_{\kappa} m\right)_{\sharp}(\omega, t)\left(\mathbb{R}^{n} \times \mathbb{R}\right) .
$$

On the other hand, $\left(\alpha_{\kappa} m\right)_{\sharp}(\omega, t)\left(\mathbb{R}^{n} \times \mathbb{R}\right)=\left(\alpha_{\kappa} m\right)_{\sharp}(\omega, t)\left(\tilde{X}_{\kappa} \times \mathbb{R}\right)$, and thus

$$
\left(\left(\alpha_{\kappa} m\right)_{\sharp}\right)_{\varepsilon}(\omega, t)\left(\tilde{X}_{\kappa} \times \mathbb{R}\right) \leq\left(\alpha_{\kappa} m\right)_{\sharp}(\omega, t)\left(\tilde{X}_{\kappa} \times \mathbb{R}\right),
$$

for any $(\omega, t) \in \Omega_{T}, \varepsilon<\bar{\varepsilon}$ and $\kappa \in \mathcal{A}$. As a consequence,

$$
\begin{gathered}
\int_{M \times \mathbb{R}} \frac{\left(\left(\alpha_{\kappa} m\right)_{\sharp}\right)_{\varepsilon}(\omega, t)(x, \xi)}{\left|h_{\kappa}(x)\right|^{1 / 2}} d \xi d V_{h}(x)=\left(\left(\alpha_{\kappa} m\right)_{\sharp}\right)_{\varepsilon}(\omega, t)\left(\tilde{X}_{\kappa} \times \mathbb{R}\right) \\
\leq\left(\alpha_{\kappa} m\right)_{\sharp}(\omega, t)\left(\tilde{X}_{\kappa} \times \mathbb{R}\right)=\int_{[0, t] \times M \times \mathbb{R}} \alpha_{\kappa}(x) m(d s, d x, d \xi),
\end{gathered}
$$

and, by summing over $\kappa \in \mathcal{A}$,

$$
\int_{M \times \mathbb{R}} m_{\varepsilon}(\omega, t)(x, \xi) d \xi d V_{h}(x) \leq \int_{[0, t] \times M \times \mathbb{R}} m(d s, d x, d \xi) .
$$

Therefore, for all $(\omega, t) \in \Omega_{T}, \varepsilon<\bar{\varepsilon}$, and $N \in \mathbb{N}$,

$$
\begin{aligned}
\int_{M \times J_{N}} m_{\varepsilon}(\omega, t)(x, \xi) d \xi d V_{h}(x) & \leq \int_{M \times \mathbb{R}} m_{\varepsilon}(\omega, t)(x, \xi) d \xi d V_{h}(x) \\
\leq \int_{[0, t] \times M \times \mathbb{R}} m(d s, d x, d \xi) & \leq \int_{[0, T] \times M \times \mathbb{R}} m(d s, d x, d \xi),
\end{aligned}
$$

which is integrable in $\omega$.

\section{Stochastic Parabolic Problem}

As a step towards constructing solutions to (1.1), we study a problem where we add to 1.1 a small diffusion term $\varepsilon \Delta_{h}(\varepsilon>0)$ involving the Laplace-Beltrami operator $\Delta_{h}$ on $(M, h)$. For a $C^{2}(M)$ function $u$,

$$
\Delta_{h} u:=\operatorname{div}_{h} \operatorname{grad}_{h} u=h^{a b}\left(\partial_{a b} u-\Gamma_{a b}^{c} \partial_{c} u\right), \quad \text { in local coordinates. }
$$

For fixed $\varepsilon>0$, we consider the parabolic problem

$$
\begin{aligned}
& d u^{\varepsilon}+\operatorname{div}_{h} f_{x}\left(u^{\varepsilon}\right) d t=\varepsilon \Delta_{h} u^{\varepsilon} d t+B\left(u^{\varepsilon}\right) d W(t), \quad x \in M, t \in(0, T), \\
& u^{\varepsilon}(0, x)=u_{0}^{\varepsilon}(x), \quad x \in M .
\end{aligned}
$$

In this section we assume the following strengthened conditions on the flux $f$, in comparison with 2.2$)$ :

$$
\begin{cases}\left|f_{x}(\xi)\right|_{h} \leq C_{0}\left(1+|\xi|^{r}\right), & |\xi| \leq L, x \in M \\ \left|f_{x}(\xi)\right|_{h} \leq C_{0}|\xi|, & |\xi|>L, x \in M\end{cases}
$$

for some positive constants $C_{0}, r, L$. Moreover, we suppose

$$
\left|f_{x}\left(\xi_{1}\right)-f_{x}\left(\xi_{2}\right)\right|_{h} \leq C_{1}\left|\xi_{1}-\xi_{2}\right|, \quad \xi_{1}, \xi_{2} \in \mathbb{R}, x \in M .
$$

for some constant $C_{1}>0$.

We wish to establish the existence and uniqueness of a solution for suitable initial data $u_{0}^{\varepsilon}$. We interpret (5.1) in the "variational framework", and look for so-called variational solutions [42]. Since $L^{2}(M, h)$ is a separable Hilbert space and $H^{1}(M, h)$ is reflexive and continuously and densely embedded in $L^{2}(M, h)$ [34, we consider the Gelfand triple

$$
V \subset H=H^{*} \subset V^{*}
$$

with $V:=H^{1}(M, h)$ and $H:=L^{2}(M, h)$. As usual, the duality pairing between $V$ and its dual $V^{*}$ will be denoted by $V^{*}\langle\cdot, \cdot\rangle_{V}$; moreover, observe that

$$
V^{*}\langle u, v\rangle_{V}=(u, v)_{H}, \quad u \in H, v \in V,
$$


where $(u, v)_{H}=\int_{M} u v d V_{h}$ is the $L^{2}(M, h)$-scalar product.

For $\varepsilon>0$ and $u \in H^{1}(M, h)$, we define $A^{\varepsilon}(u) \in V^{*}$ by

$$
V^{*}\left\langle A^{\varepsilon}(u), v\right\rangle_{V}:=\int_{M}\left(-\varepsilon \nabla u+f_{x}(u), \nabla v\right)_{h} d V_{h}(x), \quad v \in H^{1}(M, h) .
$$

We observe that, in view of 5.2 , the right-hand side is well-defined, because for any $v \in H^{1}(M, h)$ with $\|v\|_{V} \leq 1$,

$$
\begin{gathered}
\left.\right|_{V^{*}}\left\langle A^{\varepsilon}(u), v\right\rangle_{V} \mid \leq \varepsilon\|\nabla u\|_{L^{2}(M)}\|\nabla v\|_{L^{2}(M)}+C_{0}\left(1+L^{r}\right)\|\nabla v\|_{L^{1}(M)} \\
\quad+C_{0}\|u\|_{L^{2}(M)}\|\nabla v\|_{L^{2}(M)} \\
\leq \varepsilon\|\nabla u\|_{L^{2}(M)}+C_{0}\left(1+L^{r}\right)+C_{0}\|u\|_{L^{2}(M)},
\end{gathered}
$$

where in the first line we used $\|\nabla v\|_{L^{1}(M)} \leq\|\nabla v\|_{L^{2}(M)}(\operatorname{recall} \operatorname{Vol}(M, h)=1)$. We also observe that, in view of (5.3), the map $A^{\varepsilon}$ is Lipschitz from $V$ to $V^{*}$, since

$$
\begin{aligned}
\left\|A^{\varepsilon}\left(u_{1}\right)-A^{\varepsilon}\left(u_{2}\right)\right\|_{V^{*}} & =\sup _{\|V\|_{V} \leq 1} V^{*}\left\langle A^{\varepsilon}\left(u_{1}\right)-A^{\varepsilon}\left(u_{2}\right), v\right\rangle_{V} \\
& \leq \varepsilon\left\|\nabla\left(u_{1}-u_{2}\right)\right\|_{L^{2}(M)}+C_{1}\left\|u_{1}-u_{2}\right\|_{L^{2}(M)} \\
& \leq\left(\varepsilon+C_{1}\right)\left\|u_{1}-u_{2}\right\|_{H^{1}(M, h)} .
\end{aligned}
$$

In particular, $A^{\varepsilon}$ is $\mathcal{B}(V) / \mathcal{B}\left(V^{*}\right)$ measurable, and hence progressively measurable, being independent of $\omega \in \Omega$ and $t \in[0, T]$. The next two lemmas show that the assumptions $(5.2),(5.3), 2.3)$, and $(2.4)$ guarantee that we may use the variational framework of [55] to solve [5.1].

Lemma 5.1. Assume that $f=f_{x}(\xi)$ satisfies $(5.2)$ and (5.3). Then, for any $\varepsilon>0$, the map $A^{\varepsilon}: V \rightarrow V^{*}$ is hemicontinuous, weakly monotone, coercive, and bounded.

Proof. Let us check hemicontinuity. Fix $u, v, w \in H^{1}(M, h)$, and let $\lambda \in \mathbb{R}$. Then

$$
\begin{aligned}
V^{*}\left\langle A^{\varepsilon}(u+\lambda v), w\right\rangle_{V}= & \int_{M}-\varepsilon(\nabla u+\lambda \nabla v, \nabla w)_{h} d V_{h}(x) \\
& +\int_{M}\left(f_{x}(u+\lambda v), \nabla w\right)_{h} d V_{h}(x)=: I_{1}(\lambda)+I_{2}(\lambda) .
\end{aligned}
$$

Clearly, $I_{1}(\lambda)$ is linear in $\lambda$ and hence continuous. For $I_{2}(\lambda)$ we argue as follows: if $\lambda \rightarrow \lambda_{0}$, then, by smoothness of $f_{x}(\xi)$, it clearly follows that

$$
\left(f_{x}(u(x)+\lambda v(x)), \nabla w(x)\right)_{h} \stackrel{\lambda \rightarrow \lambda_{0}}{\longrightarrow}\left(f_{x}\left(u(x)+\lambda_{0} v(x)\right), \nabla w(x)\right)_{h},
$$

for every $x \in M$. In view of 5.2 , we can find a dominating function and conclude via the dominated convergence theorem. Hence, $I_{2}(\lambda)$ is continuous.

To verify weak monotonicity, we take $u, v \in H^{1}(M, h)$ and observe that

$$
\begin{aligned}
& V^{*}\left\langle A^{\varepsilon}(u)-A^{\varepsilon}(v), u-v\right\rangle_{V} \\
& \quad=-\varepsilon\|\nabla u-\nabla v\|_{L^{2}(M)}^{2}+\int_{M}\left(f_{x}(u)-f_{x}(v), \nabla u-\nabla v\right)_{h} d V_{h}(x) \\
& \quad \leq-\varepsilon\|\nabla u-\nabla v\|_{L^{2}(M)}^{2}+C_{1}\|u-v\|_{L^{2}(M)}\|\nabla u-\nabla v\|_{L^{2}(M)}
\end{aligned}
$$

by means of (5.3) and Hölder's inequality. With the help of Cauchy's inequality "with $\varepsilon$ ", we obtain

$$
\begin{aligned}
V^{*}\left\langle A^{\varepsilon}(u)-A^{\varepsilon}(v), u-v\right\rangle_{V} \leq & -\varepsilon\|\nabla u-\nabla v\|_{L^{2}(M)}^{2}+\frac{C_{1}^{2}}{2 \varepsilon}\|u-v\|_{L^{2}(M)}^{2} \\
& +\frac{\varepsilon}{2}\|\nabla u-\nabla v\|_{L^{2}(M)}^{2} \\
& \leq \frac{C_{1}^{2}}{2 \varepsilon}\|u-v\|_{L^{2}(M)}^{2} .
\end{aligned}
$$


Next we examine coercivity. For any $v \in V$, thanks to 5.2 ,

$$
\begin{aligned}
V^{*}\left\langle A^{\varepsilon}(v), v\right\rangle_{V}= & -\varepsilon\|\nabla v\|_{L^{2}(M)}^{2}+\int_{M}\left(f_{x}(v), \nabla v\right)_{h} d V_{h}(x) \\
\leq & -\varepsilon\|\nabla v\|_{L^{2}(M)}^{2}+C_{0}\left(1+L^{r}\right)\|\nabla v\|_{L^{1}(M)} \\
& +C_{0}\|V\|_{L^{2}(M)}\|\nabla v\|_{L^{2}(M)} .
\end{aligned}
$$

Once again by Cauchy's inequality "with $\varepsilon$ ",

$$
\begin{aligned}
V^{*}\left\langle A^{\varepsilon}(v), v\right\rangle_{V} \leq-\varepsilon\|\nabla v\|_{L^{2}(M)}^{2}+\frac{\varepsilon}{4}\|\nabla v\|_{L^{2}(M)}^{2}+\frac{C_{0}^{2}\left(1+L^{r}\right)^{2}}{\varepsilon} \\
\quad+\frac{\varepsilon}{4}\|\nabla v\|_{L^{2}(M)}^{2}+\frac{C_{0}^{2}}{\varepsilon}\|v\|_{L^{2}(M)}^{2} \\
=\left(\frac{C_{0}^{2}}{\varepsilon}+\frac{\varepsilon}{2}\right)\|v\|_{L^{2}(M)}^{2}-\frac{\varepsilon}{2}\|v\|_{H^{1}(M, h)}^{2}+\frac{C_{0}^{2}\left(1+L^{r}\right)^{2}}{\varepsilon} .
\end{aligned}
$$

Finally, it is clear that (5.4) implies, for any $u \in H^{1}(M, h)$,

$$
\begin{aligned}
\left\|A^{\varepsilon}(u)\right\|_{V^{*}} & \leq \varepsilon\|\nabla u\|_{L^{2}(M)}+C_{0}\left(1+L^{r}\right)+C_{0}\|u\|_{L^{2}(M)} \\
& \leq\left(\varepsilon+C_{0}\right)\|u\|_{H^{1}(M, h)}+C_{0}\left(1+L^{r}\right)
\end{aligned}
$$

This concludes the proof of the lemma.

Lemma 5.2. Assume 2.3 and 2.4 hold. Then $B$ is a Lipschitz mapping from $H^{1}(M, h)$ to $L_{2}\left(\mathfrak{U} ; L^{2}(M, h)\right)$.

Proof. From 2.5 we immediately obtain

$$
\left\|B\left(z_{1}\right)-B\left(z_{2}\right)\right\|_{L_{2}\left(\mathfrak{U} ; L^{2}(M, h)\right)}^{2} \leq D_{2}\left\|z_{1}-z_{2}\right\|_{H^{1}(M, h)}^{2},
$$

for any $z_{1}, z_{2} \in H^{1}(M, h)$; hence $B$ is Lipschitz with constant $\sqrt{D_{2}}$.

Therefore, in view of the general results in [42, Theorems $2.1 \& 2.2$, p. 1253], we infer the existence and uniqueness of a variational solution to the stochastic parabolic problem (5.1).

Theorem 5.3 (well-posedness). Suppose conditions 5.2 , (5.3), 2.3), and (2.4) hold. Fix $\varepsilon>0$, and let $u_{0}^{\varepsilon} \in L^{2}\left(\Omega ; L^{2}(M, h)\right)$ be an $\mathcal{F}_{0}$-measurable initial function. Then there exists a unique solution $u^{\varepsilon}$ to (5.1), namely a continuous $L^{2}(M, h)$ valued $\left\{\mathcal{F}_{t}\right\}_{t \in[0, T]}$-adapted process $\left(u^{\varepsilon}(t)\right)_{t \in[0, T]}$ such that $u^{\varepsilon} \in H^{1}(M, h)$ for $\mathbb{P} \otimes d t$ a.e. $(\omega, t) \in \Omega_{T}, u^{\varepsilon} \in L^{2}\left(\Omega_{T} ; H^{1}(M, h)\right) \cap L^{2}\left(\Omega_{T} ; L^{2}(M, h)\right)$, and $\mathbb{P}$-a.s.

$$
\begin{aligned}
u^{\varepsilon}(t)=u_{0}^{\varepsilon}+ & \int_{0}^{t}\left(\int_{M}\left(-\varepsilon \nabla u^{\varepsilon}(s)+f_{x}\left(u^{\varepsilon}(s)\right), \cdot\right)_{h} d V_{h}(x)\right) d s \\
& +\int_{0}^{t} B\left(u^{\varepsilon}(s)\right) d W(s), \quad t \in[0, T]
\end{aligned}
$$

where the equation is understood as equality between elements of $V^{*}$. Moreover,

$$
\mathbb{E}\left[\sup _{t \in[0, T]}\left\|u^{\varepsilon}(t)\right\|_{L^{2}(M)}^{2}\right]<\infty,
$$


and the following Itô formula holds for the square of the $L^{2}(M, h)$-norm:

$$
\begin{aligned}
& \left\|u^{\varepsilon}(t)\right\|_{L^{2}(M)}^{2}=\left\|u_{0}^{\varepsilon}\right\|_{L^{2}(M)}^{2} \\
& \quad+2 \int_{0}^{t}\left(-\varepsilon\left\|\nabla u^{\varepsilon}(s)\right\|_{L^{2}(M)}^{2}+\int_{M}\left(f_{x}\left(u^{\varepsilon}(s)\right), \nabla u^{\varepsilon}(s)\right)_{h} d V_{h}(x)\right) d s \\
& \quad+\int_{0}^{t}\left\|B\left(u^{\varepsilon}(s)\right)\right\|_{L_{2}\left(\mathfrak{U} ; L^{2}(M, h)\right)}^{2} d s \\
& \quad+2 \int_{0}^{t}\left(u^{\varepsilon}(s), B\left(u^{\varepsilon}(s)\right) d W(s)\right)_{L^{2}(M)}, \quad \mathbb{P}-\text { a.s., for any } t \in[0, T] .
\end{aligned}
$$

Remark 5.1. The expression $\int_{M}\left(-\varepsilon \nabla u^{\varepsilon}(s)+f_{x}\left(u^{\varepsilon}(s)\right), \cdot\right)_{h} d V_{h}(x)$ above denotes the linear functional on $V$ induced by $-\varepsilon \nabla u^{\varepsilon}(s)+f_{x}\left(u^{\varepsilon}(s)\right)$.

Fix any $\theta \in H^{1}(M, h)$. It is immediate to see that the following equation holds $\mathbb{P}$-a.s., for any $t \in[0, T]$ :

$$
\begin{aligned}
& \left(u^{\varepsilon}(t), \theta\right)_{L^{2}(M)} \\
& =\left(u_{0}^{\varepsilon}, \theta\right)_{L^{2}(M)}+\int_{0}^{t}\left(\int_{M}\left(-\varepsilon \nabla u^{\varepsilon}(s)+f_{x}\left(u^{\varepsilon}(s)\right), \nabla \theta\right)_{h} d V_{h}(x)\right) d s \\
& +\left(\int_{0}^{t} B\left(u^{\varepsilon}(s)\right) d W(s), \theta\right)_{L^{2}(M)} .
\end{aligned}
$$

Thus, $u^{\varepsilon}$ is a weak solution in the ordinary sense.

\section{Generalized Itô Formula}

Our aim is to establish a generalized Itô formula for weak solutions to a general class of SPDEs on Riemannian manifolds. This result will be used to derive the kinetic formulation as well as to establish a priori $L^{p}(M, h)$-estimates. An analogous formula for the Euclidean case is presented in [17] (see also [41).

The stochastic equations take the general form

$$
\begin{aligned}
& d u=F(t) d t+\operatorname{div}_{h} G(t) d t+\Delta_{h} I(t) d t+H(t) d W(t), \\
& u(0, x)=u_{0}(x),
\end{aligned}
$$

where $t \in(0, T), x \in M$, and $(M, h)$ is an $n$-dimensional $(n \geq 1)$ compact smooth Riemannian manifold, which is connected, oriented, and without boundary. The cylindrical Wiener process $W$ is defined in Section 2 ,

We assume $F \in L^{2}\left(\Omega_{T} ; L^{2}(M, h)\right)$ is predictable. Let us write $\overrightarrow{L^{2}(M, h)}$ for the separable Hilbert space of measurable vector fields on $M$ for which $|\cdot|_{h}$ belongs to $L^{2}(M, h)$. The vector field $G \in L^{2}\left(\Omega_{T} ; \overrightarrow{L^{2}(M, h)}\right)$ is assumed to be predictable. We assume $I \in L^{2}\left(\Omega_{T} ; H^{1}(M, h)\right)$ is $L^{2}(M, h)$-predictable. Moreover, we ask that $H \in L^{2}\left(\Omega_{T} ; L_{2}\left(\mathfrak{U} ; L^{2}(M, h)\right)\right)$ is predictable, and denote $H_{k}:=H e_{k}, k \in \mathbb{N}$.

Given an $\mathcal{F}_{0}$-measurable initial function $u_{0} \in L^{2}\left(\Omega ; L^{2}(M, h)\right)$, a process $u \in$ $L^{2}\left(\Omega ; C^{0}\left(0, T ; L^{2}(M, h)\right)\right) \cap L^{2}\left(\Omega_{T} ; H^{1}(M, h)\right)$ is a weak solution of 6.1) if, for any $\theta \in C^{2}(M)$, the following equation holds $\mathbb{P}$-a.s., for any $t \in[0, T]$,

$$
\begin{aligned}
(u(t), \theta)_{L^{2}(M)}=( & \left.u_{0}, \theta\right)_{L^{2}(M)}+\int_{0}^{t}\left(\int_{M} F(s) \theta(x) d V_{h}(x)\right) d s \\
& -\int_{0}^{t}\left(\int_{M}(G(s), \nabla \theta(x))_{h} d V_{h}(x)\right) d s \\
& +\int_{0}^{t} \int_{M} I(s) \Delta_{h} \theta(x) d V_{h}(x) d s+\left(\int_{0}^{t} H(s) d W(s), \theta\right)_{L^{2}(M)} .
\end{aligned}
$$


Proposition 6.1 (generalized Itô formula). Let

$$
u \in L^{2}\left(\Omega ; C^{0}\left(0, T ; L^{2}(M, h)\right)\right) \cap L^{2}\left(\Omega_{T} ; H^{1}(M, h)\right)
$$

be a weak solution of 6.1. Fix

$$
\psi \in C^{1}(M), \quad S \in C^{2}(\mathbb{R}), \quad S^{\prime \prime} \in L^{\infty}(\mathbb{R}) .
$$

Then, $\mathbb{P}$-a.s., for any $t \in[0, T]$,

$$
\begin{aligned}
& (S(u(t)), \psi)_{L^{2}(M)} \\
& =\left(S\left(u_{0}\right), \psi\right)_{L^{2}(M)}+\int_{0}^{t}\left(\int_{M} S^{\prime}(u(s)) F(s) \psi(x) d V_{h}(x)\right) d s \\
& -\int_{0}^{t}\left(\int_{M}\left(G(s), \nabla\left(S^{\prime}(u(s)) \psi(x)\right)\right)_{h} d V_{h}(x)\right) d s \\
& -\int_{0}^{t} \int_{M}\left(\nabla I(s), \nabla\left(S^{\prime}(u(s)) \psi\right)\right)_{h} d V_{h}(x) d s \\
& \quad+\left(\int_{0}^{t} S^{\prime}(u(s)) H(s) d W(s), \psi\right)_{L^{2}(M)} \\
& \quad+\frac{1}{2} \sum_{k \geq 1} \int_{0}^{t}\left(\int_{M} S^{\prime \prime}(u(s)) H_{k}^{2}(s) \psi(x) d V_{h}(x)\right) d s .
\end{aligned}
$$

Proof. The proof uses ideas from [17]. In our context further complications arise, since the underlying space is not Euclidean but a general Riemannian manifold. We use regularization by means of the heat kernel on $(M, h)$. To this end, let $\left(P_{\tau}\right)_{\tau \geq 0}$ be the heat semigroup on $L^{2}(M, h)$ and $p_{\tau}(x, y)$ its associated heat kernel (for its definition, construction and main properties, we refer to [33, 57]).

Here, we recall that $(x, y, \tau) \mapsto p_{\tau}(x, y)$ is in $C^{\infty}(M \times M \times(0, \infty))$, it is symmetric in $x$ and $y$ for any $\tau>0$, and it is positive. For a function $w \in L^{2}(M, h)$,

$$
P_{\tau} w(x)=\int_{M} p_{\tau}(x, y) w(y) d V_{h}(y), \quad x \in M, \tau>0,
$$

and $P_{\tau} w \in C^{\infty}(M)$. Moreover,

$$
P_{\tau} w \stackrel{L^{2}(M)}{\longrightarrow} w, \quad \text { as } \tau \rightarrow 0^{+},
$$

and

$$
\left\|P_{\tau} w\right\|_{L^{2}(M)} \leq\|w\|_{L^{2}(M)}, \quad \tau>0 .
$$

Finally, the following pointwise bounds hold [14]:

$$
p_{\tau}(x, y) \lesssim \tau^{-n / 2}, \quad\left|\nabla_{y} p_{\tau}(x, y)\right|_{h} \lesssim \tau^{-\frac{n+1}{2}}, \quad\left|\nabla_{y}^{2} p_{\tau}(x, y)\right|_{h} \lesssim \tau^{-\frac{n+4}{2}},
$$

for $x, y \in M$ and $\tau>0$, where $n$ is the dimension of $M$.

In the course of the proof, we will also make use of the heat kernel on forms. We hence begin by making a brief digression (for further details, we see [26, 4, 19]). Denote by $\left(\mathcal{E}_{\tau}\right)_{\tau>0}$ the de Rham-Hodge semigroup on 1-forms, associated to the de Rham-Hodge Laplacian, which by elliptic regularity has a kernel $e(\tau, \cdot, \cdot)$. More precisely, for any $\tau>0, e(\tau, \cdot, \cdot)$ is a double form on $M \times M$, such that for any 1-form $\omega \in L^{2}(M, h)$ and any $p \in M$,

$$
\left(\mathcal{E}_{\tau} \omega\right)(p)=\int_{M} e(\tau, p, q) \wedge \star_{q} \omega(q),
$$

where $\star$ is the Hodge star operator, $q$ is a typical point in $M$ and $\wedge$ is the wedge product between forms. Concretely, in a coordinate patch $\left(U,\left(x^{i}\right)\right)$ around $p$ and in a coordinate patch $\left(U^{\prime},\left(y^{j}\right)\right)$ around $q$, if we write the double form $e(\tau, \cdot, \cdot)$ as

$$
e(\tau, x, y)=\left(e(\tau, x, y)_{i j} d x^{i}\right) d y^{j}
$$


and $\omega$ as $\omega(y)=\omega_{k}(y) d y^{k}$, then the above integral becomes

$$
\left(\mathcal{E}_{\tau} \omega\right)(x)=\left(\int_{M} e(\tau, x, y)_{i j} h^{j k}(y) \omega_{k}(y) d V_{h}(y)\right) d x^{i} .
$$

For a vector field $V$, we denote by $V^{b}$ the 1 -form obtained by lowering an index via the metric $h$; analogously, for a 1 -form $\omega$, we denote by $\omega^{\sharp}$ the vector field obtained by raising an index always via the metric.

We define for a vector field $V$ the following quantity

$$
\mathcal{E}_{\tau} V:=\left(\left(\mathcal{E}_{\tau} V^{b}\right)\right)^{*}
$$

We have the following remarkable properties: for $V \in \overrightarrow{L^{2}(M, h)}$,

- $\mathcal{E}_{\tau} V$ is a smooth vector field for any $\tau>0$,

- $\mathcal{E}_{\tau} V \rightarrow V$ in $\overrightarrow{L^{2}(M, h)}$ as $\tau \rightarrow 0^{+}$,

- $\left\|\mathcal{E}_{\tau} V\right\| \underset{L^{2}(M)}{\longrightarrow} \leq\|V\| \overrightarrow{L^{2}(M)}$, for any $\tau \geq 0$.

Furthermore, in analogy with (6.3), the following local expression holds

$$
\left(\mathcal{E}_{\tau} V\right)(x)=\left(\int_{M} e(\tau, x, y)_{i j} V^{j}(y) d V_{h}(y)\right) h^{i k}(x) \partial_{k}
$$

Our aim now is to compute $\operatorname{div}_{h} \mathcal{E}_{\tau} V$, for fixed $\tau>0$. To this end, we observe that the kernel $e(\tau, x, y)$ is jointly smooth in $x$ and $y$, and that by assumption $V^{j}$ is integrable. Therefore, we are allowed to interchange differentiation and integration, to obtain

$$
\begin{aligned}
\operatorname{div}_{h} \mathcal{E}_{\tau} V(x)= & \int_{M} \partial_{k} e(\tau, x, y)_{i j} V^{j}(y) d V_{h}(y) h^{i k}(x) \\
& +\int_{M} e(\tau, x, y)_{i j} V^{j}(y) d V_{h}(y) \partial_{k} h^{i k}(x) \\
& +\Gamma_{\rho k}^{\rho}(x) \int_{M} e(\tau, x, y)_{i j} V^{j}(y) d V_{h}(y) h^{i k}(x),
\end{aligned}
$$

in local coordinates $x$ (differentiation is carried out in $x$ ).

We now fix $\tau>0$ and $x \in M$, and use $y \mapsto p_{\tau}(x, y)$ as a test function in (6.1), obtaining, $\mathbb{P}$-a.s., for any $t \in[0, T]$,

$$
\begin{aligned}
P_{\tau} u(t)(x)= & P_{\tau} u_{0}(x) \\
& +\int_{0}^{t} P_{\tau} F(s)(x) d s-\int_{0}^{t}\left(\int_{M}\left(G(s), \nabla_{y} p_{\tau}(x, y)\right)_{h} d V_{h}(y)\right) d s \\
& +\int_{0}^{t} \int_{M} I(s) \Delta_{y} p_{\tau}(x, y) d V_{h}(y) d s+\left(\int_{0}^{t} H(s) d W(s), p_{\tau}(x, \cdot)\right)_{L^{2}(M)},
\end{aligned}
$$

where we have employed the stochastic Fubini theorem [13, Thm 4.33]. The last term may be written as

$$
\left(\int_{0}^{t} H(s) d W(s), p_{\tau}(x, \cdot)\right)_{L^{2}(M)}=\sum_{k \geq 1} \int_{0}^{t} P_{\tau} H_{k}(s)(x) d \beta_{k}(s) .
$$

In view of the predictability and integrability assumptions made on $F, G, I, H$, we are allowed to apply the classical Itô formula [13, Thm. 4.32] to $S\left(P_{\tau} u(t)\right)$. We 
multiply the result by $\psi(x)$, obtaining, $\mathbb{P}$-a.s., for any $t \in[0, T], x \in M$, and $\tau>0$,

$$
\begin{aligned}
S\left(P_{\tau} u(t)\right) \psi=S & \left.P_{\tau} u_{0}\right) \psi+\int_{0}^{t} S^{\prime}\left(P_{\tau} u(s)\right) \psi P_{\tau} F(s) d s \\
& -\int_{0}^{t} S^{\prime}\left(P_{\tau} u(s)\right) \psi\left(\int_{M}\left(G(s), \nabla_{y} p_{\tau}(\cdot, y)\right)_{h} d V_{h}(y)\right) d s \\
& +\int_{0}^{t} S^{\prime}\left(P_{\tau} u(s)\right) \psi\left(\int_{M} I(s) \Delta_{y} p_{\tau}(\cdot, y) d V_{h}(y)\right) d s \\
& +\sum_{k \geq 1} \int_{0}^{t} S^{\prime}\left(P_{\tau} u(s)\right) \psi P_{\tau} H_{k}(s) d \beta_{k}(s) \\
& +\frac{1}{2} \sum_{k \geq 1} \int_{0}^{t} S^{\prime \prime}\left(P_{\tau} u(s)\right) \psi\left(P_{\tau} H_{k}(s)\right)^{2} d s .
\end{aligned}
$$

In view of

$$
|S(\xi)| \leq C\left(1+\xi^{2}\right), \quad\left|S^{\prime}(\xi)\right| \leq C(1+|\xi|), \quad \xi \in \mathbb{R},
$$

the pointwise estimates 6.5 on $p_{\tau}$ and the integrability assumptions on $u$, we can integrate the expression above w.r.t. $d V_{h}(x)$, arriving at

$$
\begin{aligned}
\int_{M} S & \left(P_{\tau} u(t)\right) \psi(x) d V_{h}(x)=\int_{M} S\left(P_{\tau} u_{0}\right) \psi(x) d V_{h}(x) \\
& +\int_{0}^{t} \int_{M} S^{\prime}\left(P_{\tau} u(s)\right) \psi(x) P_{\tau} F(s) d V_{h}(x) d s \\
& -\int_{0}^{t} \int_{M} S^{\prime}\left(P_{\tau} u(s)\right) \psi(x)\left(\int_{M}\left(G(s), \nabla_{y} p_{\tau}(x, y)\right)_{h} d V_{h}(y)\right) d V_{h}(x) d s \\
& +\int_{0}^{t} \int_{M} S^{\prime}\left(P_{\tau} u(s)\right) \psi(x)\left(\int_{M} I(s) \Delta_{y} p_{\tau}(x, y) d V_{h}(y)\right) d V_{h}(x) d s \\
& +\int_{M} \sum_{k \geq 1} \int_{0}^{t} S^{\prime}\left(P_{\tau} u(s)\right) \psi(x) P_{\tau} H_{k}(s) d \beta_{k}(s) d V_{h}(x) \\
& +\frac{1}{2} \sum_{k \geq 1} \int_{0}^{t} \int_{M} S^{\prime \prime}\left(P_{\tau} u(s)\right) \psi(x)\left(P_{\tau} H_{k}(s)\right)^{2} d V_{h}(x) d s,
\end{aligned}
$$

$\mathbb{P}$-a.s., for any $t \in[0, T]$, and $\tau>0$. Moreover, we can interchange the integrals appearing in the formula above thanks to the Fubini and stochastic Fubini theorems.

The aim is to prove that, as $\tau \rightarrow 0^{+}$, each term in 6.8 converges $\mathbb{P}$-a.s. to its corresponding term in 6.2). Let us begin with the term on the left hand side: in view of 6.7$)$, it follows that $S(u(t)) \in L^{1}(M, h), \mathbb{P}$-a.s., for any $t \in[0, T]$. Moreover, since $\left|S\left(r_{1}\right)-S\left(r_{2}\right)\right| \leq C\left(\left(1+\left|r_{2}\right|\right)\left|r_{1}-r_{2}\right|+\left|r_{1}-r_{2}\right|^{2}\right)$, for any $r_{1}, r_{2} \in \mathbb{R}$,

$$
\begin{aligned}
& \int_{M}\left|S\left(P_{\tau} u(t)\right)-S(u(t))\right||\psi(x)| d V_{h}(x) \\
& \leq C\|\psi\|_{L^{\infty}} \int_{M}\left|P_{\tau} u(t)-u(t)\right|(1+|u(t)|) d V_{h}(x) \\
& \quad+C\|\psi\|_{L^{\infty}} \int_{M}\left|P_{\tau} u(t)-u(t)\right|^{2} d V_{h}(x) \\
& \leq C\left\|P_{\tau} u(t)-u(t)\right\|_{L^{2}(M)}\|1+|u(t)|\|_{L^{2}(M)}+C\left\|P_{\tau} u(t)-u(t)\right\|_{L^{2}(M)}^{2},
\end{aligned}
$$

and thus, by (6.4), we obtain

$$
\int_{M} S\left(P_{\tau} u(t)\right) \psi(x) d V_{h}(x) \stackrel{\tau \downarrow 0}{\rightarrow} \int_{M} S(u(t)) \psi(x) d V_{h}(x), \quad \mathbb{P} \text {-a.s., } t \in[0, T] .
$$


Similarly, $\mathbb{P}$-a.s., $\int_{M} S\left(P_{\tau} u_{0}\right) \psi(x) d V_{h}(x) \rightarrow \int_{M} S\left(u_{0}\right) \psi(x) d V_{h}(x)$.

In view of (6.7), $S^{\prime}(u(t)) \in L^{2}(M, h), \mathbb{P}$-a.s., $t \in[0, T]$, and

$$
\begin{aligned}
\int_{0}^{t} \int_{M}\left|S^{\prime}\left(P_{\tau} u(t)\right) P_{\tau} F(s)-S^{\prime}(u(t)) F(s)\right||\psi(x)| d V_{h}(x) d s \\
\leq\|\psi\|_{L^{\infty}} \int_{0}^{t} \int_{M}\left|S^{\prime}\left(P_{\tau} u(s)\right)-S^{\prime}(u(s))\right|\left|P_{\tau} F(s)\right| d V_{h}(x) d s \\
\quad+\|\psi\|_{L^{\infty}} \int_{0}^{t} \int_{M}\left|S^{\prime}(u(s))\right|\left|P_{\tau} F(s)-F(s)\right| d V_{h}(x) d s .
\end{aligned}
$$

Since $\left|S^{\prime}\left(r_{1}\right)-S^{\prime}\left(r_{2}\right)\right| \leq\left\|S^{\prime \prime}\right\|_{L^{\infty}}\left|r_{1}-r_{2}\right|$, for any $r_{1}, r_{2} \in \mathbb{R}$, we infer

$$
\begin{aligned}
& \int_{0}^{t} \int_{M}\left|S^{\prime}\left(P_{\tau} u(t)\right) P_{\tau} F(s)-S^{\prime}(u(t)) F(s)\right||\psi(x)| d V_{h}(x) d s \\
& \leq C \int_{0}^{t} \int_{M}\left|P_{\tau} u(s)-u(s)\right|\left|P_{\tau} F(s)\right| d V_{h}(x) d s \\
& \quad+C \int_{0}^{t} \int_{M}\left(1+|(u(s) \mid)| P_{\tau} F(s)-F(s) \mid d V_{h}(x) d s\right. \\
& \leq C \int_{0}^{t}\left\|P_{\tau} u(s)-u(s)\right\|_{L^{2}(M)}\|F(s)\|_{L^{2}(M)} d s \\
&+C \int_{0}^{t}\left\|P_{\tau} F(s)-F(s)\right\|_{L^{2}(M)} d s \\
&+C \int_{0}^{t}\|u(s)\|_{L^{2}(M)}\left\|P_{\tau} F(s)-F(s)\right\|_{L^{2}(M)} d s,
\end{aligned}
$$

where we have used Hölder's inequality to obtain the second inequality. Since $\left\|P_{\tau} u(s)-u(s)\right\|_{L^{2}(M)} \rightarrow 0$, P -a.s., for each $s$, and $\left\|P_{\tau} F(s)-F(s)\right\|_{L^{2}(M)} \rightarrow 0$ for $\mathbb{P} \otimes d t$-a.e. $(\omega, s)$, as $\tau \rightarrow 0^{+}$, we can use the dominated convergence theorem to conclude that the terms above go to zero. Thus, $\mathbb{P}$-a.s., for any $t \in[0, T]$,

$$
\int_{0}^{t} \int_{M} S^{\prime}\left(P_{\tau} u(s)\right) \psi(x) P_{\tau} F(s) d V_{h}(x) d s \stackrel{\tau \downarrow 0}{\rightarrow} \int_{0}^{t} \int_{M} S^{\prime}(u(s)) \psi(x) F(s) d V_{h}(x) d s .
$$

Let us now deal with the last term in 6.8. We have

$$
\begin{aligned}
\sum_{k \geq 1} \int_{0}^{t} \int_{M}\left|S^{\prime \prime}\left(P_{\tau} u(s)\right)\left(P_{\tau} H_{k}(s)\right)^{2}-S^{\prime \prime}(u(s)) H_{k}^{2}(s)\right||\psi(x)| d V_{h}(x) d s \\
\leq\|\psi\|_{L^{\infty}} \sum_{k \geq 1} \int_{0}^{t} \int_{M}\left|S^{\prime \prime}\left(P_{\tau} u(s)\right)\right|\left|\left(P_{\tau} H_{k}(s)\right)^{2}-H_{k}^{2}(s)\right| d V_{h}(x) d s \\
\quad+\|\psi\|_{L^{\infty}} \sum_{k \geq 1} \int_{0}^{t} \int_{M}\left|S^{\prime \prime}\left(P_{\tau} u(s)\right)-S^{\prime \prime}(u(s))\right|\left|H_{k}^{2}(s)\right| d V_{h}(x) d s \\
=: I_{1}+I_{2} .
\end{aligned}
$$

By the general fact $\left\|a^{2}-b^{2}\right\|_{L^{1}} \leq\|a-b\|_{L^{2}}\|a+b\|_{L^{2}}$,

$$
\begin{aligned}
I_{1} & \leq\|\psi\|_{L^{\infty}}\left\|S^{\prime \prime}\right\|_{L^{\infty}} \sum_{k \geq 1} \int_{0}^{t}\left\|\left(P_{\tau} H_{k}(s)\right)^{2}-H_{k}^{2}(s)\right\|_{L^{1}(M)} d s \\
& \leq C \sum_{k \geq 1} \int_{0}^{t}\left\|P_{\tau} H_{k}(s)-H_{k}(s)\right\|_{L^{2}(M)}\left\|P_{\tau} H_{k}(s)+H_{k}(s)\right\|_{L^{2}(M)} d s .
\end{aligned}
$$

We have $\left\|P_{\tau} H_{k}(s)-H_{k}(s)\right\|_{L^{2}(M)} \rightarrow 0$ as $\tau \rightarrow 0^{+}$for $\mathbb{P} \otimes d t$-a.e. $(\omega, s)$. Furthermore, $\left\|P_{\tau} H_{k}(s) \pm H_{k}(s)\right\|_{L^{2}(M)} \leq 2\left\|H_{k}(s)\right\|_{L^{2}(M)}$ for $\mathbb{P} \otimes d t$-a.e. $(\omega, s)$ and (by 
assumption) $H \in L^{2}\left(\Omega_{T} ; L_{2}\left(\mathfrak{U} ; L^{2}(M, h)\right)\right)$. Therefore, by the dominated convergence theorem, $I_{1} \rightarrow 0$ as $\tau \rightarrow 0^{+}$, $\mathbb{P}$-a.s., $t \in[0, T]$.

For the term $I_{2}$ we proceed in this way: since $P_{\tau} u(s) \rightarrow u(s)$ in $L^{2}(M, h)$ as $\tau \rightarrow 0^{+}, \mathbb{P}$-a.s., for a.e. $s$, we infer by Lemma 8.1 that

$$
S^{\prime \prime}\left(P_{\tau} u(s)\right) \stackrel{\tau \downarrow 0}{\rightarrow} S^{\prime \prime}(u(s)) \text { in } L^{1}(M, h), \quad \mathbb{P} \text {-a.s., } s \in[0, t] .
$$

Let $\left(\tau_{j}\right)_{j}$ be a sequence tending to 0 as $j \rightarrow \infty$. We extract a subsequence $\left(\tau_{j_{i}}\right)_{i}$. By the reverse dominated convergence theorem, we can extract a further subsequence (not relabelled) such that $\left|S^{\prime \prime}\left(P_{\tau_{j_{i}}} u(s)(x)\right)-S^{\prime \prime}(u(s)(x))\right| \rightarrow 0$ as $i \rightarrow \infty, \mathbb{P}$-a.s., for a.e. $x \in M$ and $s \in[0, t]$. Thus, $\sum_{k}\left|H_{k}^{2}(s)(x)\right|\left|S^{\prime \prime}\left(P_{\tau_{j_{i}}} u(s)(x)\right)-S^{\prime \prime}(u(s)(x))\right|$ converges to zero. Besides, $\sum_{k}\left|H_{k}^{2}(s)(x)\right|\left|S^{\prime \prime}\left(P_{\tau_{j_{i}}} u(s)(x)\right)-S^{\prime \prime}(u(s)(x))\right|$ can be bounded by $2\left\|S^{\prime \prime}\right\|_{L^{\infty}} \sum_{k \geq 1} H_{k}^{2}(s)(x)$, P-a.s., for a.e. $x \in M$ and $s \geq 0$. In view of our assumptions, the latter term belongs to $L^{1}(M, h), \mathbb{P}$-a.s., for a.e. $s \geq 0$. By the dominated convergence theorem, we infer $\sum_{k}\left|H_{k}^{2}(s)\right|\left|S^{\prime \prime}\left(P_{\tau_{j_{i}}} u(s)\right)-S^{\prime \prime}(u(s))\right| \rightarrow$ 0 in $L^{1}(M, h)$ as $i \rightarrow \infty, \mathbb{P}$-a.s., for a.e. $s \in[0, t]$. This is true for the original sequence $\left(\tau_{j}\right)_{j}$ as well. In other words,

$$
\left\|\sum_{k \geq 1}\left|H_{k}^{2}(s)\right|\left|S^{\prime \prime}\left(P_{\tau_{j_{i}}} u(s)\right)-S^{\prime \prime}(u(s))\right|\right\|_{L^{1}(M)} \stackrel{\tau \downarrow 0}{\rightarrow} 0, \quad \mathbb{P} \text {-a.s., } s \in[0, t] .
$$

This last quantity is bounded by $2\left\|S^{\prime \prime}\right\|_{L^{\infty}}\|H(s)\|_{L_{2}\left(\mathfrak{U} ; L^{2}(M, h)\right)}^{2}$, which is $\mathbb{P} \otimes d t$ integrable by assumption. By dominated convergence, we eventually conclude that

$$
I_{2} \stackrel{\tau \downarrow 0}{\rightarrow} 0, \quad \mathbb{P} \text {-a.s., } t \in[0, T] .
$$

Next we consider the stochastic term. First of all, by the Burkholder-DavisGundy inequality 2.6 and 6.7),

$$
\begin{aligned}
& \mathcal{I}:=\mathbb{E} \sup _{0 \leq t \leq T}\left|\sum_{k \geq 1} \int_{0}^{t} \int_{M} S^{\prime}(u(s)) H_{k}(s) \psi(x) d V_{h}(x) d \beta_{k}(s)\right| \\
& \leq C \mathbb{E}\left(\int_{0}^{T} \sum_{k \geq 1}\left|\int_{M} S^{\prime}(u(s)) H_{k}(s) \psi(x) d V_{h}(x)\right|^{2} d s\right)^{\frac{1}{2}} \\
& \leq\left(\int_{0}^{T}\left\|S^{\prime}(u(s))\right\|_{L^{2}(M)}^{2}\|H(s)\|_{L_{2}\left(\mathfrak{U} ; L^{2}(M, h)\right)}^{2} d s\right)^{\frac{1}{2}} \\
& \leq C \mathbb{E}\left(\int_{0}^{T}\left(1+\|u(s)\|_{L^{2}(M)}^{2}\right)\|H(s)\|_{L_{2}\left(\mathfrak{U} ; L^{2}(M, h)\right)}^{2} d s\right)^{\frac{1}{2}} \\
& \leq C \mathbb{E}\left(\int_{0}^{T}\|u(s)\|_{L^{2}(M)}^{2}\|H(s)\|_{L_{2}\left(\mathfrak{U} ; L^{2}(M, h)\right)}^{2} d s\right)^{\frac{1}{2}} \\
&+C \mathbb{E}\left(\int_{0}^{T}\|H(s)\|_{L_{2}\left(\mathfrak{U} ; L^{2}(M, h)\right)}^{2} d s\right)^{\frac{1}{2}},
\end{aligned}
$$


where we have used the inequality $\sqrt{x+y} \leq \sqrt{x}+\sqrt{y}$, valid for $x, y \geq 0$. Therefore, by Hölder's inequality and the integrability assumptions on $u$ and $H$,

$$
\begin{aligned}
& \mathcal{I} \leq C \mathbb{E}\left(\sup _{0 \leq s \leq T}\|u(s)\|_{L^{2}(M)}^{2} \int_{0}^{T}\|H(s)\|_{L_{2}\left(\mathfrak{U} ; L^{2}(M, h)\right)}^{2} d s\right)^{\frac{1}{2}} \\
& +C\left(\mathbb{E} \int_{0}^{T}\|H(s)\|_{L_{2}\left(\mathfrak{U} ; L^{2}(M, h)\right)}^{2} d s\right)^{\frac{1}{2}} \\
& \leq C\left(\mathbb{E} \int_{0}^{T}\|H(s)\|_{L_{2}\left(\mathfrak{U} ; L^{2}(M, h)\right)}^{2} d s\right)^{\frac{1}{2}}\left[1+\left(\mathbb{E} \sup _{0 \leq s \leq T}\|u(s)\|_{L^{2}(M)}^{2}\right)^{\frac{1}{2}}\right],
\end{aligned}
$$

which is finite. Thus, the stochastic integral in $\sqrt{6.2}$ is well defined. Therefore, arguing as above,

$$
\begin{aligned}
\mathbb{E} \sup _{0 \leq t \leq T}\left|\sum_{k \geq 1} \int_{0}^{t} \int_{M}\left[S^{\prime}\left(P_{\tau} u(s)\right) P_{\tau} H_{k}(s)-S^{\prime}(u(s)) H_{k}(s)\right] \psi(x) d V_{h}(x) d \beta_{k}(s)\right| \\
\leq C \mathbb{E}\left(\int_{0}^{T}\left\|S^{\prime}\left(P_{\tau} u(s)\right)-S^{\prime}(u(s))\right\|_{L^{2}(M)}^{2}\left\|P_{\tau} H(s)\right\|_{L_{2}\left(\mathfrak{U} ; L^{2}(M, h)\right)}^{2} d s\right)^{\frac{1}{2}} \\
\quad+C \mathbb{E}\left(\int_{0}^{T}\left\|S^{\prime}(u(s))\right\|_{L^{2}(M)}^{2}\left\|P_{\tau} H(s)-H(s)\right\|_{L_{2}\left(\mathfrak{U} ; L^{2}(M, h)\right)}^{2} d s\right)^{\frac{1}{2}} \\
=: J_{1}+J_{2} .
\end{aligned}
$$

We have

$$
\left.J_{1} \leq C \mathbb{E}\left(\int_{0}^{T} \| P_{\tau} u(s)\right)-u(s)\left\|_{L^{2}(M)}^{2}\right\| H(s) \|_{L_{2}\left(\mathfrak{U} ; L^{2}(M, h)\right)}^{2} d s\right)^{\frac{1}{2}} .
$$

The term $\left.\| P_{\tau} u(s)\right)-u(s)\left\|_{L^{2}(M)}^{2}\right\| H(s) \|_{L_{2}\left(\mathfrak{U} ; L^{2}(M, h)\right)}^{2}$ is dominated by the quantity $2 \sup _{0 \leq s \leq T}\|u(s)\|_{L^{2}(M)}^{2}\|H(s)\|_{L_{2}\left(\mathfrak{U} ; L^{2}(M, h)\right)}^{2}$ for $\mathbb{P} \otimes d s$ a.e. $(\omega, s)$, which is integrable in time, $\mathbb{P}$-almost surely. Moreover, $\left.\| P_{\tau} u(s)\right)-u(s) \|_{L^{2}(M)}^{2} \rightarrow 0$ as $\tau \rightarrow 0^{+}$, for $\mathbb{P} \otimes d s$ a.e. $(\omega, s)$. Hence, by the dominated convergence theorem,

$$
\left.\left(\int_{0}^{T} \| P_{\tau} u(s)\right)-u(s)\left\|_{L^{2}(M)}^{2}\right\| H(s) \|_{L_{2}\left(\mathfrak{U} ; L^{2}(M, h)\right)}^{2} d s\right)^{\frac{1}{2}} \stackrel{\tau \downarrow 0}{\rightarrow} 0, \quad \mathbb{P} \text {-almost surely. }
$$

This last quantity is bounded uniformly in $\tau$ by

$$
2\left(\sup _{0 \leq s \leq T}\|u(s)\|_{L^{2}(M)}^{2}\right)^{\frac{1}{2}}\left(\int_{0}^{T}\|H(s)\|_{L_{2}\left(\mathfrak{U} ; L^{2}(M, h)\right)}^{2} d s\right)^{\frac{1}{2}}, \quad \mathbb{P} \text {-a.s. }
$$

which is $\mathbb{P}$-integrable by assumption. Applying again the dominated convergence theorem, the conclusion is that

$$
J_{1} \rightarrow 0, \text { as } \tau \rightarrow 0^{+}
$$


Because $\left\|P_{\tau} H(s)-H(s)\right\|_{L_{2}\left(\mathfrak{U} ; L^{2}(M, h)\right)}^{2} \rightarrow 0$ for $\mathbb{P} \otimes d s$ a.e. $(\omega, s)$, the same conclusion holds true for $J_{2}$ as well. Therefore, up to a subsequence, we obtain

$$
\begin{aligned}
\sum_{k \geq 1} \int_{0}^{t} & \int_{M} S^{\prime}\left(P_{\tau} u(s)\right) P_{\tau} H_{k}(s) \psi(x) d V_{h}(x) d \beta_{k}(s) \\
& \stackrel{\tau \downarrow 0}{\longrightarrow}\left(\int_{0}^{t} S^{\prime}(u(s)) H(s) d W(s), \psi\right)_{L^{2}(M)}, \quad \mathbb{P} \text {-almost surely. }
\end{aligned}
$$

We are going to examine the remaining terms:

$$
A(\tau):=-\int_{0}^{t} \int_{M} S^{\prime}\left(P_{\tau} u(s)(x)\right) \psi(x)\left(\int_{M}\left(G(s), \nabla_{y} p_{\tau}(x, y)\right)_{h} d V_{h}(y)\right) d V_{h}(x) d s
$$

and

$$
B(\tau):=\int_{0}^{t} \int_{M} S^{\prime}\left(P_{\tau} u(s)\right) \psi(x)\left(\int_{M} I(s) \Delta_{y} p_{\tau}(x, y) d V_{h}(y)\right) d V_{h}(x) d s .
$$

We temporarily assume that

- for $\mathbb{P} \otimes d t$-a.e. $(\omega, t) \in \Omega_{T}, G(\omega, t)$ is a $C^{1}$ vector field (so that $\operatorname{div}_{h} G$ exists for $\mathbb{P} \otimes d t$-a.e. $\left.(\omega, t) \in \Omega_{T}\right)$,

- $\operatorname{div}_{h} G \in L^{2}\left(\Omega_{T} ; L^{2}(M, h)\right)$.

- for $\mathbb{P} \otimes d t$-a.e. $(\omega, t) \in \Omega_{T}, I(\omega, t)$ is in $C^{2}(M)$ (so that $\Delta_{h} I$ exists for $\mathbb{P} \otimes d t$-a.e. $\left.(\omega, t) \in \Omega_{T}\right)$, and

- $\Delta_{h} I \in L^{2}\left(\Omega_{T} ; L^{2}(M, h)\right)$.

As a result we are now allowed to integrate by parts in the expressions above.

For the first term we obtain, $\mathbb{P}$-a.s.,

$$
\begin{aligned}
A_{\tau} & =\int_{0}^{t} \int_{M} S^{\prime}\left(P_{\tau} u(s)(x)\right) \psi(x)\left(\int_{M} \operatorname{div}_{h, y} G(s, y) p_{\tau}(x, y) d V_{h}(y)\right) d V_{h}(x) d s \\
& =\int_{0}^{t} \int_{M} S^{\prime}\left(P_{\tau} u(s)(x)\right) \psi(x) P_{\tau}\left(\operatorname{div}_{h, y} G(s)\right)(x) d V_{h}(x) d s .
\end{aligned}
$$

For this reason, arguing exactly as was done with the $F$-term, we conclude that this quantity converges as $\tau \rightarrow 0^{+}$to

$$
\begin{aligned}
\int_{0}^{t} & \int_{M} S^{\prime}(u(s)(x)) \psi(x)\left(\operatorname{div}_{h, y} G(s)\right)(x) d V_{h}(x) d s \\
& =\int_{0}^{t} \int_{M} S^{\prime}(u(s)(x)) \psi(x) \operatorname{div}_{h} G(s)(x) d V_{h}(x) d s
\end{aligned}
$$

$\mathbb{P}$-a.s., $t \in[0, T]$. Observe that, in view of the integrability assumptions made on $u$ and (6.7), the chain rule for Sobolev functions implies $S^{\prime}(u(s)) \psi \in H^{1}(M, h)$ for $\mathbb{P} \otimes d t$ a.e. $(\omega, s) \in \Omega_{T}$, a fact that enables us to integrate by parts in the last expression. Summarizing, under the additional smoothness assumptions made above on $G$, we have proved that

$$
A_{\tau} \stackrel{\tau \downarrow 0}{\rightarrow}-\int_{0}^{t} \int_{M}\left(G(s), \nabla\left(S^{\prime}(u(s)) \psi\right)\right)_{h} d V_{h}(x) d s, \quad \mathbb{P} \text {-a.s., } t \in[0, T] .
$$

A completely analogous result holds for $B_{\tau}$ :

$$
B_{\tau} \stackrel{\tau \downarrow 0}{\rightarrow}-\int_{0}^{t} \int_{M}\left(\nabla I(s), \nabla\left(S^{\prime}(u(s)) \psi\right)\right)_{h} d V_{h}(x) d s, \quad \mathbb{P} \text {-a.s., } t \in[0, T] .
$$

Recapping the story so far, the generalized Itô formula holds under additional smoothness assumptions on $G$ and $I$. To establish the formula in the general case, we must apply a regularization procedure, using the heat kernel for $I$ and the heat kernel on forms for $G$. 
Assume $G \in L^{2}\left(\Omega_{T} ; \overrightarrow{L^{2}(M, h)}\right), I \in L^{2}\left(\Omega_{T} ; H^{1}(M, h)\right)$ are $L^{2}(M, h)$-predictable. Consider an arbitrary sequence $\left(\tau_{l}\right)_{l} \subset(0,1)$ with $\tau_{l} \rightarrow 0^{+}$as $l \rightarrow \infty$. We start by regularizing $G$. Indeed, $\mathcal{E}_{\tau_{l}} G(s)$ is a smooth vector field for $\mathbb{P} \otimes d t$ a.e. $(\omega, s) \in \Omega_{T}$. Obviously, $\mathcal{E}_{\tau_{l}} G \in L^{2}\left(\Omega_{T} ; \overrightarrow{L^{2}(M, h)}\right)$ is predictable. Finally, $\operatorname{div}_{h} \mathcal{E}_{\tau_{l}} G$ exists and is in $L^{2}(M, h)$ for $\mathbb{P} \otimes d t$ a.e. $(\omega, s) \in \Omega_{T}$. To show that $\operatorname{div}_{h} \mathcal{E}_{\tau_{l}} G$ is in $L^{2}\left(\Omega_{T} ; L^{2}(M, h)\right)$ we proceed like this: for any $\gamma \in C^{\infty}(M)$, consider the $\mathbb{R}$-valued stochastic process

$$
\Omega_{T} \ni(\omega, t) \mapsto \Lambda_{\gamma}=\left(\operatorname{div}_{h} \mathcal{E}_{\tau_{l}} G(t), \gamma\right)_{L^{2}(M)}=-\left(\mathcal{E}_{\tau_{l}} G(t), \nabla \gamma\right) \overrightarrow{L^{2}(M)} .
$$

Since (by assumption) $G$ is predictable and $\mathcal{E}_{\tau_{l}}$ is a continuous from $\overrightarrow{L^{2}(M, h)}$ to itself, it follows that $\mathcal{E}_{\tau_{l}} G$ is predictable. Moreover, by continuity of the scalar product, we immediately infer that $\Lambda_{\gamma}$ is predictable for any $\gamma \in C^{\infty}(M)$ as well. Because $L^{2}(M, h)$ is separable and $\overline{C^{\infty}(M)}{ }^{L^{2}(M)}=L^{2}(M, h)$, the image of $C^{\infty}(M)$ under the Riesz isomorphism of $L^{2}(M, h)$ is a norming set, and thus, in view of 20. Cor. 4, Ch. 2], we conclude that $\operatorname{div}_{h} \mathcal{E}_{\tau_{l}} G$ is predictable. In particular, it is $\mathcal{F} \otimes \mathcal{B}([0, T]) / \mathcal{B}\left(L^{2}(M)\right)$ measurable. Given a Banach space $X$, we recall that a subset $G \subset X^{*}$ is norming if $\|x\|=\sup _{x^{*} \in G}\left|\left\langle x^{*}, x\right\rangle\right| /\left\|x^{*}\right\|$ for each $x \in X$.

By means of $(6.6)$, we may write locally, for $\mathbb{P} \otimes d t$-a.e. $(\omega, s) \in \Omega_{T}$,

$$
\begin{aligned}
\operatorname{div}_{h} & \mathcal{E}_{\tau_{l}} G(s)(x)=\int_{M} \partial_{k} e\left(\tau_{l}, x, y\right)_{i j} G^{j}(s, y) d V_{h}(y) h^{i k}(x) \\
& +\int_{M} e\left(\tau_{l}, x, y\right)_{i j} G^{j}(s, y) d V_{h}(y)\left(\partial_{k} h^{i k}(x)+\Gamma_{\rho k}^{\rho}(x) h^{i k}(x)\right) .
\end{aligned}
$$

Hence, for each fixed $l \in \mathbb{N}$, there is a constant $C\left(M, h, \tau_{l}\right)>0$ such that

$$
\left|\operatorname{div}_{h} \mathcal{E}_{\tau_{l}} G(s)(x)\right| \leq C\left(M, h, \tau_{l}\right)\|G(s)\|_{\overrightarrow{L^{2}(M, h)}},
$$

for $\mathbb{P} \otimes d t$-a.e. $(\omega, s) \in \Omega_{T}$ and all $x$ in the coordinate patch. Therefore,

$$
\int_{M}\left|\operatorname{div}_{h} \mathcal{E}_{\tau_{l}} G(s)(x)\right|^{2} d V_{h}(x) \leq C(M, h)\|G(s)\| \frac{2}{L^{2}(M, h)},
$$

for $\mathbb{P} \otimes d t$ a.e. $(\omega, s) \in \Omega_{T}$, and the required integrability is a consequence of $G \in L^{2}\left(\Omega_{T} ; L^{2}(M, h)\right)$.

Concerning the $I$ term, we apply $P_{\tau_{l}}$ to it. Exploiting the general fact

$$
\left\|P_{\tau_{l}} w\right\|_{H^{1}(M, h)} \leq\|w\|_{H^{1}(M, h)}, \quad w \in H^{1}(M, h),
$$

and arguing as before, we can show that $P_{\tau_{l}} I \in L^{2}\left(\Omega_{T} ; H^{1}(M, h)\right)$ and $P_{\tau_{l}} I, \Delta_{h} P_{\tau_{l}} I$ are $L^{2}(M)$-predictable. To verify the required integrability, we need access to an explicit expression for $\Delta_{h} P_{\tau_{l}} I$. According to [33, Theorem 7.20], locally it holds

$$
\begin{aligned}
\Delta_{h} P_{\tau_{l}} I(s)(x)= & h^{a b}(x)\left(\partial_{a b} P_{\tau_{l}} I(s)(x)-\Gamma_{a b}^{c}(x) \partial_{c} P_{\tau_{l}} I(s)(x)\right) \\
= & h^{a b}(x) \int_{M} \partial_{a b} p_{\tau_{l}}(x, y) I(s, y) d V_{h}(y) \\
& \quad-h^{a b}(x) \Gamma_{a b}^{c}(x) \int_{M} \partial_{c} p_{\tau_{l}}(x, y) I(s, y) d V_{h}(y) \\
= & h^{a b}(x) \int_{M}\left(\partial_{a b} p_{\tau_{l}}(x, y)-\Gamma_{a b}^{c}(x) \partial_{c} p_{\tau_{l}}(x, y)\right) I(s, y) d V_{h}(y) \\
= & \int_{M} \Delta_{h, x} p_{\tau_{l}}(x, y) I(s, y) d V_{h}(y) .
\end{aligned}
$$

Hence, in view of the integrability assumptions made on $I$ and repeating earlier arguments, we infer $\Delta_{h} P_{\tau_{l}} I \in L^{2}\left(\Omega_{T} ; L^{2}(M, h)\right)$. 
We are thus allowed to apply Itô's formula 6.2 with $\mathcal{E}_{\tau_{l}} G$ and $P_{\tau_{l}} I$, which will hold for any $t \in[0, T]$ and all $\omega \in \Omega_{l}$ for some $\Omega_{l}$ with $\mathbb{P}\left(\Omega_{l}\right)=1$. Set

$$
\Omega_{0}:=\bigcap_{l \in \mathbb{N}} \Omega_{l}, \quad \mathbb{P}\left(\Omega_{0}\right)=1,
$$

and fix $\omega \in \Omega_{0}$ and $t \in[0, T]$. To conclude the proof, we are required to check that

$$
\int_{0}^{t} \int_{M}\left(\mathcal{E}_{\tau_{l}} G(s), \nabla\left(S^{\prime}(u(s)) \psi\right)\right)_{h} d V_{h} d s \stackrel{\tau \downarrow 0}{\rightarrow} \int_{0}^{t} \int_{M}\left(G(s), \nabla\left(S^{\prime}(u(s)) \psi\right)\right)_{h} d V_{h} d s
$$

and

$\int_{0}^{t} \int_{M}\left(\nabla P_{\tau_{l}} I(s), \nabla\left(S^{\prime}(u(s)) \psi\right)\right)_{h} d V_{h} d s \stackrel{\tau \downarrow 0}{\rightarrow} \int_{0}^{t} \int_{M}\left(\nabla I(s), \nabla\left(S^{\prime}(u(s)) \psi\right)\right)_{h} d V_{h} d s$.

Regarding the first convergence claim,

$$
\begin{aligned}
& \int_{0}^{t} \int_{M}\left|\left(\mathcal{E}_{\tau_{l}} G(s)-G(s), \nabla\left(S^{\prime}(u(s)) \psi\right)\right)_{h}\right| d V_{h} d s \\
& \leq \int_{0}^{t} \int_{M}\left|\left(\mathcal{E}_{\tau_{l}} G(s)-G(s), \nabla u(s)\right)_{h} S^{\prime \prime}(u(s)) \psi\right| d V_{h} d s \\
& \quad+\int_{0}^{t} \int_{M}\left|\left(\mathcal{E}_{\tau_{l}} G(s)-G(s), \nabla \psi\right)_{h} S^{\prime}(u(s))\right| d V_{h} d s \\
& \leq\|\psi\|_{L^{\infty}}\left\|S^{\prime \prime}\right\|_{L^{\infty}} \int_{0}^{t}\left\|\mathcal{E}_{\tau_{l}} G(s)-G(s)\right\|_{L^{2}(M)}\|u(s)\|_{H^{1}(M, h)} d s \\
& \quad+C(M, h)\|\nabla \psi\|_{L^{\infty}} \int_{0}^{t}\left\|\mathcal{E}_{\tau_{l}} G(s)-G(s)\right\|_{L^{2}(M)}\left\|S^{\prime}(u(s))\right\|_{L^{2}(M)} d s \\
& \leq C \int_{0}^{t}\left\|\mathcal{E}_{\tau_{l}} G(s)-G(s)\right\|_{L^{2}(M)}^{\longrightarrow}\left(1+\|u(s)\|_{H^{1}(M, h)}\right) d s .
\end{aligned}
$$

The integrand in the last term converges to zero as $l \rightarrow \infty$, for $\mathbb{P} \otimes d t$-a.e. $(\omega, s)$, and it is dominated by $C(M, h)\|G(s)\|_{\overrightarrow{L^{2}(M)}}\left(1+\|u(s)\|_{H^{1}(M, h)}\right)$, which is integrable on $\Omega_{T}$. Thus, the integral converges to zero by the dominated convergence theorem.

In a completely analogous way, using the fact that $P_{\tau_{l}} w \rightarrow w$ in $H^{1}(M, h)$, for $w \in H^{1}(M, h)$, we see that also the second convergence claim holds. This concludes the proof of Proposition 6.1.

\section{Existence RESUlT}

In this section we will prove the existence part of Theorem 3.2 . We will achieve this by arguing that (weak) solutions to the parabolic problem (5.1) converge as $\varepsilon \rightarrow 0$ to a generalized kinetic solution of 1.1 . In view of the already established rigidity/uniqueness results (cf. Section 4), the existence claim will follow from this.

Complying with Section 5 we will without loss generality assume that the flux $f$ satisfies the conditions $(5.2)$ and $(5.3)$. These conditions can be replaced by (2.2) at the expense of carrying out an additional approximation (and convergence) argument. We leave the details to the interested reader.

7.1. Kinetic formulation of parabolic SPDE. We start by writing the kinetic equation (in weak form) linked to the parabolic problem (5.1).

Proposition 7.1 (kinetic formulation). Fix $\varepsilon>0$ and $u_{0} \in L^{\infty}(M, h)$. Let $u^{\varepsilon}$ be the unique weak solution of (5.1), with initial data $\left.u^{\varepsilon}\right|_{t=0}=u_{0}$, given by Theorem 
5.3. Then $\rho^{\varepsilon}:=\mathbb{I}_{u^{\varepsilon}>\xi}$ satisfies

$$
\begin{aligned}
\int_{0}^{T} & \int_{M} \int_{\mathbb{R}} \rho^{\varepsilon} \partial_{t} \psi d \xi d V_{h}(x) d t+\int_{M} \int_{\mathbb{R}} \rho_{0} \psi(0, x, \xi) d \xi d V_{h}(x) \\
& +\int_{0}^{T} \int_{M} \int_{\mathbb{R}} \rho^{\varepsilon}\left(f_{x}^{\prime}(\xi), \nabla \psi\right)_{h} d \xi d V_{h}(x) d t \\
& +\varepsilon \int_{0}^{T} \int_{M} \int_{\mathbb{R}} \rho^{\varepsilon} \Delta_{h} \psi d \xi d V_{h}(x) d t=m^{\varepsilon}\left(\partial_{\xi} \psi\right) \\
& -\sum_{k \geq 1} \int_{0}^{T} \int_{M} \int_{\mathbb{R}} g_{k}(x, \xi) \psi \nu_{\omega, t, x}^{\varepsilon}(d \xi) d V_{h}(x) d \beta_{k}(t) \\
& -\frac{1}{2} \int_{0}^{T} \int_{M} \int_{\mathbb{R}} \partial_{\xi} \psi G^{2}(x, \xi) \nu_{\omega, t, x}^{\varepsilon}(d \xi) d V_{h}(x) d t, \quad \text { P-almost surely, }
\end{aligned}
$$

$\forall \psi \in C_{c}^{\infty}([0, T) \times M \times \mathbb{R})$, where $\rho_{0}:=\mathbb{I}_{u_{0}>\xi}$ and, for $\Psi \in C_{b}^{0}([0, T] \times M \times \mathbb{R})$,

$$
\nu_{\omega, t, x}^{\varepsilon}=\delta_{u^{\varepsilon}(\omega, t, x)}, m^{\varepsilon}(\Psi)=\int_{0}^{T} \int_{M} \int_{\mathbb{R}} \varepsilon\left|\nabla u^{\varepsilon}(t)\right|_{h}^{2} \Psi(t, x, \xi) \delta_{u^{\varepsilon}(t)}(d \xi) d V_{h}(x) d t .
$$

Proof. As the proof is fairly standard, we keep the level of detail at a minimum. We use the generalized Itô formula (cf. Proposition 6.1) with $F \equiv 0, G(s)=-f_{x}\left(u^{\varepsilon}(s)\right.$ ), $I(s)=\varepsilon u^{\varepsilon}(s), H(s)=B\left(u^{\varepsilon}(s)\right), \psi \in \mathcal{D}(M)$, and the nonlinear function $S(\xi)=$ $\int_{-\infty}^{\xi} \lambda(r) d r, \lambda \in \mathcal{D}(\mathbb{R})$. Taking into account 2.1), the result is the following equation which holds $\mathbb{P}$-a.s., for any $t \in[0, T]$ :

$$
\begin{aligned}
\int_{M}\left\langle\rho^{\varepsilon}(t), \lambda\right\rangle_{\mathcal{D}^{\prime}(\mathbb{R})} \psi(x) d V_{h}(x)-\int_{M}\left\langle\rho_{0}, \lambda\right\rangle_{\mathcal{D}^{\prime}(\mathbb{R})} \psi(x) d V_{h}(x) \\
=\int_{0}^{t} \int_{M}\left\langle\partial_{\xi}\left(\varepsilon\left|\nabla u^{\varepsilon}(s)\right|_{h}^{2} \delta_{u^{\varepsilon}(s)}\right), \lambda\right\rangle_{\mathcal{D}^{\prime}(\mathbb{R})} \psi(x) d V_{h}(x) d s \\
-\varepsilon \int_{0}^{t} \int_{M}\left(\nabla\left\langle\rho^{\varepsilon}(s), \lambda\right\rangle_{\mathcal{D}^{\prime}(\mathbb{R})}, \nabla \psi\right)_{h} d V_{h}(x) d s \\
-\int_{0}^{t} \int_{M} \operatorname{div}_{h}\left\langle\rho^{\varepsilon}(s), f_{x}^{\prime}(\cdot) \lambda\right\rangle_{\mathcal{D}^{\prime}(\mathbb{R})} \psi(x) d V_{h}(x) d s \\
\quad+\sum_{k \geq 1} \int_{0}^{t} \int_{M}\left\langle\delta_{u^{\varepsilon}(s)}, \lambda g_{k}(x, \cdot)\right\rangle_{\mathcal{D}^{\prime}(\mathbb{R})} \psi(x) d V_{h}(x) d \beta_{k}(s) \\
-\frac{1}{2} \int_{0}^{t} \int_{M}\left\langle\partial_{\xi}\left(\delta_{u^{\varepsilon}(s)} G^{2}(x, \cdot)\right), \lambda\right\rangle_{\mathcal{D}^{\prime}(\mathbb{R})} \psi(x) d V_{h}(x) d s .
\end{aligned}
$$

As the topological tensor product $\mathcal{D}(M) \otimes \mathcal{D}(\mathbb{R})$ is a dense subspace of $\mathcal{D}(M \times \mathbb{R})$ (cf. [19, p. 38]), the formula remains valid for any $\psi \in \mathcal{D}(M \times \mathbb{R})$. Indeed, after some simple manipulations, the following equation holds $\mathbb{P}$-a.s., for any $t \in[0, T]$, and for any $\psi \in \mathcal{D}(M \times \mathbb{R})$ :

$$
\begin{aligned}
& \int_{M \times \mathbb{R}} \rho^{\varepsilon}(t) \psi(x, \xi) d \xi d V_{h}(x)-\int_{M \times \mathbb{R}} \rho_{0} \psi(x, \xi) d \xi d V_{h}(x) \\
&=- \int_{0}^{t} \int_{M} \varepsilon\left|\nabla u^{\varepsilon}(s)\right|_{h}^{2}\left(\partial_{\xi} \psi\right)\left(x, u^{\varepsilon}(s)\right) d V_{h}(x) d s \\
&+\varepsilon \int_{0}^{t} \int_{M \times \mathbb{R}} \rho^{\varepsilon}(s) \Delta_{h} \psi d \xi d V_{h}(x) d s \\
&+\int_{0}^{t} \int_{M \times \mathbb{R}} \rho^{\varepsilon}(s)\left(f_{x}^{\prime}(\xi) \nabla \psi(x, \xi)\right)_{h} d \xi d V_{h}(x) d s
\end{aligned}
$$




$$
\begin{aligned}
& +\sum_{k \geq 1} \int_{0}^{t} \int_{M} g_{k}\left(x, u^{\varepsilon}(s)\right) \psi\left(x, u^{\varepsilon}(s)\right) d V_{h}(x) d \beta_{k}(s) \\
& +\frac{1}{2} \int_{0}^{t} \int_{M}\left(\partial_{\xi} \psi\right)\left(x, u^{\varepsilon}(s)\right) G^{2}\left(x, u^{\varepsilon}(s)\right) d V_{h}(x) d s .
\end{aligned}
$$

We multiply this equation by $\partial_{t} \theta$ and integrate over $[0, T]$, with $\theta \in \mathcal{D}(-T, T)$. The stochastic term is handled using "integration by parts". The final result is

$$
\begin{gathered}
\int_{0}^{T} \int_{M \times \mathbb{R}} \rho^{\varepsilon}(t) \psi(x, \xi) \partial_{t} \theta(t) d \xi d V_{h}(x) d t+\int_{M \times \mathbb{R}} \rho_{0} \psi(x, \xi) \theta(0) d \xi d V_{h}(x) \\
=\int_{0}^{T} \int_{M} \varepsilon\left|\nabla u^{\varepsilon}(t)\right|_{h}^{2}\left(\partial_{\xi} \psi\right)\left(x, u^{\varepsilon}(t)\right) \theta(t) d V_{h}(x) d t \\
-\varepsilon \int_{0}^{T} \int_{M \times \mathbb{R}} \rho^{\varepsilon}(t) \Delta_{h} \psi \theta(t) d \xi d V_{h}(x) d t \\
-\int_{0}^{T} \int_{M \times \mathbb{R}} \rho^{\varepsilon}(t)\left(f_{x}^{\prime}(\xi) \nabla \psi(x, \xi)\right)_{h} \theta(t) d \xi d V_{h}(x) d t \\
\quad-\sum_{k \geq 1} \int_{0}^{T} \int_{M} g_{k}\left(x, u^{\varepsilon}(t)\right) \psi\left(x, u^{\varepsilon}(t)\right) \theta(t) d V_{h}(x) d \beta_{k}(t) \\
-\frac{1}{2} \int_{0}^{T} \int_{M}\left(\partial_{\xi} \psi\right)\left(x, u^{\varepsilon}(t)\right) G^{2}\left(x, u^{\varepsilon}(t)\right) \theta(t) d V_{h}(x) d t .
\end{gathered}
$$

By density of tensor products, this equation continue to hold for any test function $\psi \in \mathcal{D}((-T, T) \times M \times \mathbb{R})$. Finally, if we are given a function $\psi \in \mathcal{D}([0, T) \times M \times \mathbb{R})$, we extend it to a function belonging to $\mathcal{D}((-T, T) \times M \times \mathbb{R})$ using the Whitney extension theorem. The equation holds for this extension too.

7.2. A priori $L^{p}$ estimates. Our goal is to use the generalized Itô formula in Proposition 6.1 to establish an $\varepsilon$-independent $L^{p}$ bound on the weak solution to the parabolic SPDE (5.1). Before we can do that we need two auxiliary lemmas, which make use of the geometric compatibility condition (2.1).

Lemma 7.2. Suppose $f=f_{x}(\xi)$ is a smooth geometry-compatible vector field on $M$, smoothly depending on $\xi \in \mathbb{R}$. For $u \in C^{1}(M)$ and $S \in C^{2}(\mathbb{R})$ with $S^{\prime \prime} \in L^{\infty}(\mathbb{R})$,

$$
\int_{M}\left(f_{x}(u), \nabla S^{\prime}(u)\right)_{h} d V_{h}(x)=\int_{M}\left(f_{x}(u), \nabla u\right)_{h} S^{\prime \prime}(u) d V_{h}(x)=0 .
$$

Proof. Following [7], we define

$$
F_{x}(\xi):=\int_{0}^{\xi} S^{\prime}(w) \partial_{w} f_{x}(w) d w \in T_{x} M, \quad x \in M, \xi \in \mathbb{R} .
$$

The geometry compatibility condition 2.1) implies the following pointwise identity:

$$
S^{\prime}(u(x)) \operatorname{div}_{h} f_{x}(u(x))=\operatorname{div}_{h} F_{x}(u(x)), \quad x \in M .
$$

Therefore, the divergence theorem implies

$$
\begin{aligned}
& \int_{M}\left(f_{x}(u), \nabla u\right)_{h} S^{\prime \prime}(u) d V_{h}(x)=\int_{M}\left(f_{x}(u), \nabla S^{\prime}(u)\right)_{h} d V_{h}(x) \\
& =-\int_{M} S^{\prime}(u(x)) \operatorname{div}_{h} f_{x}(u(x)) d V_{h}(x)=-\int_{M} \operatorname{div}_{h} F_{x}(u(x)) d V_{h}(x)=0 .
\end{aligned}
$$

Lemma 7.3. Suppose $f=f_{x}(\xi)$ is a smooth geometry-compatible vector field on $M$, smoothly depending on $\xi \in \mathbb{R}$, such that $\sqrt{5.2}$ and 5.3 hold. The conclusion of Lemma 7.2 remains valid for any $u \in H^{1}(M, h)$. 
Proof. By definition of $H^{1}(M, h)$, we can find a sequence $\left(u_{m}\right)_{m} \subset C^{1}(M)$ such that $u_{m} \rightarrow u$ in $H^{1}(M, h)$ as $m \rightarrow \infty$. By Lemma 7.2.

$$
\int_{M}\left(f_{x}\left(u_{m}\right), \nabla u_{m}\right)_{h} S^{\prime \prime}\left(u_{m}\right) d V_{h}(x)=0, \quad m \in \mathbb{N} .
$$

Let us write this equation as

$$
\begin{aligned}
0= & \int_{M}\left(f_{x}\left(u_{m}\right), \nabla u_{m}\right)_{h} S^{\prime \prime}\left(u_{m}\right) d V_{h}(x) \\
= & \int_{M}\left(f_{x}\left(u_{m}\right)-f_{x}(u), \nabla u_{m}\right)_{h} S^{\prime \prime}\left(u_{m}\right) d V_{h}(x) \\
& \quad+\int_{M}\left(f_{x}(u), \nabla u_{m}-\nabla u\right)_{h} S^{\prime \prime}\left(u_{m}\right) d V_{h}(x) \\
& \quad+\int_{M}\left(f_{x}(u), \nabla u\right)_{h}\left(S^{\prime \prime}\left(u_{m}\right)-S^{\prime \prime}(u)\right) d V_{h}(x) \\
& +\int_{M}\left(f_{x}(u), \nabla u\right)_{h} S^{\prime \prime}(u) d V_{h}(x) \\
=: A_{1} & +A_{2}+A_{3}+\int_{M}\left(f_{x}(u), \nabla u\right)_{h} S^{\prime \prime}(u) d V_{h}(x) .
\end{aligned}
$$

The lemma follows if we can show that $A_{1}, A_{2}, A_{3} \rightarrow 0$ as $m \rightarrow \infty$.

Clearly,

$$
\left|A_{1}\right| \leq C_{1}\left\|S^{\prime \prime}\right\|_{L^{\infty}}\left\|u-u_{m}\right\|_{L^{2}(M)} \sup _{m}\left\|\nabla u_{m}\right\|_{L^{2}(M)},
$$

where $C_{1}$ is the constant in $(5.3)$. Therefore, $A_{1} \rightarrow 0$ as $m \rightarrow \infty$.

In a similar way, employing (5.2),

$$
\begin{aligned}
\left|A_{2}\right| \leq C_{0}\left\|S^{\prime \prime}\right\|_{L^{\infty}}((1+ & \left.L^{r}\right)\left\|\nabla u_{m}-\nabla u\right\|_{L^{1}(M)} \\
& \left.+\|u\|_{L^{2}(M)}\left\|\nabla u-\nabla u_{m}\right\|_{L^{2}(M)}\right),
\end{aligned}
$$

and hence $A_{2} \rightarrow 0$ as $m \rightarrow \infty$.

For the remaining term, we argue like this:

$$
\begin{aligned}
\left|A_{3}\right| \leq & C_{0} \int_{M}\left(1+L^{r}\right)|\nabla u|_{h}\left|S^{\prime \prime}\left(u_{m}\right)-S^{\prime \prime}(u)\right| d V_{h}(x) \\
& +C_{0} \int_{M}|u||\nabla u|_{h}\left|S^{\prime \prime}\left(u_{m}\right)-S^{\prime \prime}(u)\right| d V_{h}(x) \\
\leq & C_{0}\left(1+L^{r}\right)\|\nabla u\|_{L^{2}(M)}\left\|S^{\prime \prime}\left(u_{m}\right)-S^{\prime \prime}(u)\right\|_{L^{2}(M)} \\
& +C_{0} \int_{M}|u||\nabla u|_{h}\left|S^{\prime \prime}\left(u_{m}\right)-S^{\prime \prime}(u)\right| d V_{h}(x) .
\end{aligned}
$$

By invoking Lemma 8.1 (in the appendix), $S^{\prime \prime}\left(u_{m}\right) \rightarrow S^{\prime \prime}(u)$ in $L^{p}(M, h)$ as $m \rightarrow \infty$, for any $p \in[1, \infty)$. Therefore,

$$
\left|A_{3}\right| \leq o(1)+C_{0} \int_{M}|u||\nabla u|_{h}\left|S^{\prime \prime}\left(u_{m}\right)-S^{\prime \prime}(u)\right| d V_{h}(x),
$$

where $o(1) \rightarrow 0$ as $m \rightarrow \infty$. Let $p_{n}$ and $q_{n}$ be defined by

$$
p_{n}= \begin{cases}2, & \text { if } n=1 \\ \geq 1, & \text { if } n=2 \\ n, & \text { if } n>2\end{cases}
$$

and $\frac{1}{q_{n}}+\frac{1}{2}+\frac{1}{p_{n}}=1$, where $n$ is the dimension of the manifold. Therefore, by the generalized Hölder inequality and the Sobolev embedding,

$$
\left|A_{3}\right| \leq o(1)+C_{0}\|u\|_{L^{q_{n}}(M)}\|\nabla u\|_{L^{2}(M)}\left\|S^{\prime \prime}\left(u_{m}\right)-S^{\prime \prime}(u)\right\|_{L^{p_{n}(M)}},
$$


and thus $A_{3} \rightarrow 0$ as $m \rightarrow \infty$, again thanks to Lemma 8.1

Fix $\varepsilon>0$, and consider $\mathcal{F}_{0}$-measurable initial datum $u_{0}^{\varepsilon} \in L^{2}\left(\Omega ; L^{2}(M, h)\right)$. Denote by $u^{\varepsilon}$ the corresponding weak (variational) solution given by Theorem 5.3 . In what follows, we derive some basic energy estimates that will allow us to show (weak) convergence of $u^{\varepsilon}$ as $\varepsilon \rightarrow 0$.

Proposition 7.4. There exists an $\varepsilon$-independent constant $K>0$ such that

$$
\mathbb{E} \sup _{0 \leq t \leq T}\left\|u^{\varepsilon}(t)\right\|_{L^{2}(M)}^{2}+\varepsilon \mathbb{E} \int_{0}^{T}\left\|\nabla u^{\varepsilon}(t)\right\|_{L^{2}(M)}^{2} d t \leq K\left(1+\mathbb{E}\left\|u_{0}^{\varepsilon}\right\|_{L^{2}(M)}^{2}\right) .
$$

Proof. From equation (5.5), it follows that

$$
\begin{aligned}
\mathbb{E} & \left\|u^{\varepsilon}(t)\right\|_{L^{2}(M)}^{2}=\mathbb{E}\left\|u_{0}^{\varepsilon}\right\|_{L^{2}(M)}^{2} \\
& +2 \int_{0}^{t} \mathbb{E}\left(-\varepsilon\left\|\nabla u^{\varepsilon}(s)\right\|_{L^{2}(M)}^{2}+\int_{M}\left(f_{x}\left(u^{\varepsilon}(s)\right), \nabla u^{\varepsilon}(s)\right)_{h} d V_{h}(x)\right) d s \\
& +\int_{0}^{t} \mathbb{E}\left\|B\left(u^{\varepsilon}(s)\right)\right\|_{L_{2}\left(\mathfrak{U} ; L^{2}(M, h)\right)}^{2} d s, \quad t \in[0, T]
\end{aligned}
$$

For additional details, see [55, Remark 4.2.8]. Because $u^{\varepsilon}$ belongs to $H^{1}(M, h)$ for $\mathbb{P} \otimes d t$-a.e. $(\omega, s) \in \Omega_{T}$, it follows from Lemma 7.3 with $S(\xi)=\xi^{2}$ that

$$
\int_{0}^{t} \mathbb{E} \int_{M}\left(f_{x}\left(u^{\varepsilon}(s)\right), \nabla u^{\varepsilon}(s)\right)_{h} d V_{h}(x) d s=0 .
$$

Using this and 2.3 , we obtain

$$
\begin{aligned}
\mathbb{E}\left\|u^{\varepsilon}(t)\right\|_{L^{2}(M)}^{2} & \leq \mathbb{E}\left\|u_{0}^{\varepsilon}\right\|_{L^{2}(M)}^{2}+\int_{0}^{t} \mathbb{E}\left\|B\left(u^{\varepsilon}(s)\right)\right\|_{L_{2}\left(\mathfrak{U} ; L^{2}(M, h)\right)}^{2} d s \\
& \leq \mathbb{E}\left\|u_{0}^{\varepsilon}\right\|_{L^{2}(M)}^{2}+D_{1} \int_{0}^{t} \mathbb{E}\left(1+\left\|u^{\varepsilon}(s)\right\|_{L^{2}(M)}^{2}\right) d s \\
& =\mathbb{E}\left\|u_{0}^{\varepsilon}\right\|_{L^{2}(M)}^{2}+D_{1} t+\int_{0}^{t} D_{1} \mathbb{E}\left\|u^{\varepsilon}(s)\right\|_{L^{2}(M)}^{2} d s .
\end{aligned}
$$

Note that $t \mapsto \mathbb{E}\left\|u^{\varepsilon}(t)\right\|_{L^{2}(M)}^{2}$ is continuous, and that $t \mapsto \mathbb{E}\left\|u_{0}^{\varepsilon}\right\|_{L^{2}(M)}^{2}+D_{1} t$ is non-decreasing and positive. Hence, an application of Gronwall's inequality yields

$$
\mathbb{E}\left\|u^{\varepsilon}(t)\right\|_{L^{2}(M)}^{2} \leq e^{D_{1} t}\left(\mathbb{E}\left\|u_{0}^{\varepsilon}\right\|_{L^{2}(M)}^{2}+D_{1} t\right), \quad t \in[0, T] .
$$

Let us now return to (5.5). Using once more (2.3) and Lemma 7.3 , taking the supremum and then the expectation, and making use of the elementary inequality $\sup _{t} \alpha(t)+\sup _{t} \beta(t) \leq 2 \sup _{t} \gamma(t)$ valid for positive functions $\alpha(t), \beta(t)$ satisfying $\alpha(t)+\beta(t) \leq \gamma(t)$, we obtain

$$
\begin{aligned}
\mathbb{E} \sup _{0 \leq t \leq T}\left\|u^{\varepsilon}(t)\right\|_{L^{2}(M)}^{2}+2 \varepsilon \mathbb{E} \int_{0}^{T}\left\|\nabla u^{\varepsilon}(s)\right\|_{L^{2}(M)}^{2} d s \\
\leq 2 \mathbb{E}\left\|u_{0}^{\varepsilon}\right\|_{L^{2}(M)}^{2}+2 D_{1} T+2 D_{1} \int_{0}^{T} \mathbb{E}\left\|u^{\varepsilon}(s)\right\|_{L^{2}(M)}^{2} d s \\
+4 \mathbb{E} \sup _{0 \leq t \leq T}\left|\sum_{k \geq 1} \int_{0}^{t} \int_{M} u^{\varepsilon}(s) g_{k}\left(x, u^{\varepsilon}(s)\right) d V_{h}(x) d \beta_{k}(s)\right| .
\end{aligned}
$$

By the Burkholder-Davis-Gundy inequality (2.6), arguing as in the proof of Proposition 6.1 and employing Cauchy's inequality, we can bound the last term by

$$
\frac{1}{2} \mathbb{E} \sup _{0 \leq t \leq T}\left\|u^{\varepsilon}(t)\right\|_{L^{2}(M)}^{2}+C\left[1+\int_{0}^{T} \mathbb{E}\left\|u^{\varepsilon}(s)\right\|_{L^{2}(M)}^{2} d s\right],
$$


where $C$ depends on $D_{1}, T$ but not $\varepsilon, C_{0}, r, L, C_{1}, D_{2}$. Thus, for a new constant $C$,

$$
\begin{aligned}
& \frac{1}{2} \mathbb{E} \sup _{0 \leq t \leq T}\left\|u^{\varepsilon}(t)\right\|_{L^{2}(M)}^{2}+2 \varepsilon \mathbb{E} \int_{0}^{T}\left\|\nabla u^{\varepsilon}(s)\right\|_{L^{2}(M)}^{2} d s \\
& \quad \leq 2 \mathbb{E}\left\|u_{0}^{\varepsilon}\right\|_{L^{2}(M)}^{2}+C\left(1+\int_{0}^{T} \mathbb{E}\left\|u^{\varepsilon}(s)\right\|_{L^{2}(M)}^{2} d s\right) .
\end{aligned}
$$

Combining this with 7.2 , we arrive at the claim (7.1).

With similar reasoning, this time using our generalized Itô formula 6.2 , it is possible to derive a related bound for the $L^{p}$-norm.

Proposition 7.5. Fix $p \in(2, \infty)$. There exists an $\varepsilon$-independent constant $K_{p}>0$ such that

$$
\begin{aligned}
& \mathbb{E} \sup _{0 \leq t \leq T}\left\|u^{\varepsilon}(t)\right\|_{L^{p}(M)}^{p}+\varepsilon \mathbb{E} \int_{0}^{T} \int_{M}\left|u^{\varepsilon}(t)\right|^{p-2}\left|\nabla u^{\varepsilon}(t)\right|_{h}^{2} d V_{h}(x) d t \\
& \quad \leq K_{p}\left(1+\mathbb{E}\left\|u_{0}^{\varepsilon}\right\|_{L^{p}(M)}^{p}\right) .
\end{aligned}
$$

Remark 7.1. The bound 7.3 implies

$$
\mathbb{E}\left(\underset{t \in[0, T]}{\operatorname{ess} \sup _{M}} \int_{\mathbb{R}} \int_{\mathbb{R}}|\xi|^{p} \nu_{\omega, t, x}^{\varepsilon}(d \xi) d V_{h}(x)\right) \leq C_{p}
$$

for some $\varepsilon$-independent constant $C_{p}>0$, where the "Dirac delta" Young measure $\nu_{\omega, t, x}^{\varepsilon}$ is defined in Proposition 7.1

Proof. To show the assertion we would like to employ the generalized Itô formula with $\psi \equiv 1$ and nonlinear function $S(\xi)=|\xi|^{p}$. Unfortunately this cannot be done directly as the second derivative of $S$ is not bounded. We are therefore forced to utilize a suitable approximation of $S$, following an idea from [18.

For $m \in \mathbb{N}$, define $S_{m} \in C^{2}(\mathbb{R})$ by

$$
S_{m}(\xi)=\left\{\begin{array}{ll}
|\xi|^{p}, & |\xi| \leq m \\
m^{p-2}\left\{\frac{p(p-1)}{2} \xi^{2}-p(p-2) m|\xi|+\frac{(p-1)(p-2)}{2} m^{2}\right\}, & |\xi|>m
\end{array} .\right.
$$

The following elementary properties can be easily verified:

$$
\begin{aligned}
& \left|\xi S_{m}^{\prime}(\xi)\right| \leq p S_{m}(\xi), \\
& \left|S_{m}^{\prime}(\xi)\right| \leq p\left(1+S_{m}(\xi)\right), \\
& \left|S_{m}^{\prime}(\xi)\right| \leq|\xi| S_{m}^{\prime \prime}(\xi), \\
& \xi^{2} S_{m}^{\prime \prime}(\xi) \leq p(p-1) S_{m}(\xi), \\
& S_{m}^{\prime \prime}(\xi) \leq p(p-1)\left(1+S_{m}(\xi)\right) .
\end{aligned}
$$

Before applying Proposition 6.1, we make a few preliminary observations. Since $u^{\varepsilon}$ is $L^{2}(M, h)$-predictable and $B$ is Lipschitz on $L^{2}(M, h)$, it follows that $B\left(u^{\varepsilon}\right)$ is $L_{2}\left(\mathfrak{U} ; L^{2}(M, h)\right)$-predictable. By means of $(5.3)$, we see that also $f_{x}\left(u^{\varepsilon}\right)$ is $\overrightarrow{L^{2}(M, h)}$ predictable. Moreover, if we utilize test functions in $(5.6)$ that are in $C^{2}(M)$, we are allowed to integrate by parts the term involving $\nabla u^{\varepsilon}$, obtaining

$$
\int_{0}^{t} \int_{M} \varepsilon u^{\varepsilon}(s) \Delta_{h} \theta d V_{h}(x) d s .
$$

Setting $F \equiv 0, G(s):=-f_{x}\left(u^{\varepsilon}(s)\right), I(s):=\varepsilon u^{\varepsilon}(s)$, and $H(s):=B\left(u^{\varepsilon}(s)\right)$, we see that all the assumptions in Proposition 6.1 are satisfied. As a result, we can 
apply the generalized Itô formula with $S_{m}(\cdot)$ and $\psi \equiv 1$, with the result that

$$
\begin{aligned}
& \int_{M} S_{m}\left(u^{\varepsilon}(t)\right) d V_{h}(x)=\int_{M} S_{m}\left(u_{0}^{\varepsilon}\right) d V_{h}(x) \\
& +\int_{0}^{t} \int_{M}\left(-\varepsilon \nabla u^{\varepsilon}(s)+f_{x}\left(u^{\varepsilon}(s)\right), \nabla S_{m}^{\prime}\left(u^{\varepsilon}(s)\right)\right)_{h} d V_{h}(x) d s \\
& \quad+\left(\int_{0}^{t} S_{m}^{\prime}\left(u^{\varepsilon}(s)\right) B\left(u^{\varepsilon}(s)\right) d W(s), 1\right)_{L^{2}(M)} \\
& \quad+\frac{1}{2} \sum_{k \geq 1} \int_{0}^{t} \int_{M} S_{m}^{\prime \prime}\left(u^{\varepsilon}(s)\right) g_{k}^{2}\left(x, u^{\varepsilon}(s)\right) d V_{h}(x) d s
\end{aligned}
$$

$\mathbb{P}$-a.s., for any $t \in[0, T]$.

In view of Lemma 7.3 , also in the present case the integral involving the flux $f$ vanishes. Furthermore, since $S_{m}^{\prime \prime} \geq 0$, we see that

$$
\begin{aligned}
& \int_{0}^{t} \int_{M}\left(-\varepsilon \nabla u^{\varepsilon}(s), \nabla S_{m}^{\prime}\left(u^{\varepsilon}(s)\right)\right)_{h} d V_{h}(x) d s \\
& \quad=-\varepsilon \int_{0}^{t} \int_{M}\left|\nabla u^{\varepsilon}(s)\right|_{h}^{2} S_{m}^{\prime \prime}\left(u^{\varepsilon}(s)\right) d V_{h}(x) d s \leq 0 .
\end{aligned}
$$

By making use of 7.4 and 2.3,

$$
\begin{aligned}
& \frac{1}{2} \int_{0}^{t} \int_{M} S_{m}^{\prime \prime}\left(u^{\varepsilon}(s)\right) G^{2}\left(x, u^{\varepsilon}(s)\right) d V_{h}(x) d s \\
& \quad \leq \frac{D_{1}}{2} \int_{0}^{t} \int_{M} S_{m}^{\prime \prime}\left(u^{\varepsilon}(s)\right)\left(1+\left|u^{\varepsilon}(s)\right|^{2}\right) d V_{h}(x) d s \\
& \quad \leq D_{1} p(p-1) \int_{0}^{t} \int_{M}\left(\frac{1}{2}+S_{m}\left(u^{\varepsilon}(s)\right)\right) d V_{h}(x) d s
\end{aligned}
$$

Utilizing our findings in 7.5 and then taking the expectation, we obtain, for any $t \in[0, T]$,

$$
\begin{aligned}
\mathbb{E} \int_{M} S_{m}\left(u^{\varepsilon}(t)\right) d V_{h}(x) \leq \mathbb{E} \int_{M} S_{m}\left(u_{0}^{\varepsilon}\right) d V_{h}(x) \\
+\mathbb{E} \sum_{k \geq 1} \int_{0}^{t} \int_{M} S_{m}^{\prime}\left(u^{\varepsilon}(s)\right) g_{k}\left(x, u^{\varepsilon}(s)\right) d V_{h}(x) d \beta_{k}(s) \\
\quad+D_{1} p(p-1) \frac{t}{2}+\int_{0}^{t} D_{1} p(p-1) \mathbb{E} \int_{M} S_{m}\left(u^{\varepsilon}(s)\right) d V_{h}(x) d s .
\end{aligned}
$$

In view of the Burkholder-Davis-Gundy inequality (2.6), arguing as in the proof of Proposition 7.4 it follows that the second term on the right-hand side of the inequality can be bounded as follows:

$$
\begin{aligned}
& \mathbb{E} \sup _{0 \leq t \leq T}\left|\sum_{k \geq 1} \int_{0}^{t} \int_{M} S_{m}^{\prime}\left(u^{\varepsilon}(s)\right) g_{k}\left(x, u^{\varepsilon}(s)\right) d V_{h}(x) d \beta_{k}(s)\right| \\
& \quad \leq C\left(1+\mathbb{E} \sup _{0 \leq t \leq T}\left\|u^{\varepsilon}(t)\right\|_{L^{2}(M)}^{2}\right),
\end{aligned}
$$

where the constant $C$ depends on $D_{1}, T, p$ but not $m, \varepsilon, C_{0}, r, L, C_{1}, D_{2}$. From Proposition 7.4 and the simple fact that $\left\|u^{\varepsilon}\right\|_{L^{2}(M)}^{2} \leq 1+\left\|u^{\varepsilon}\right\|_{L^{p}(M)}^{p}(p>2)$, the last term may be bounded by

$$
C\left(1+\mathbb{E}\left\|u_{0}^{\varepsilon}\right\|_{L^{p}(M)}^{p}\right)
$$


for some new constant $C$.

Noting that $S_{m}(\xi) \leq|\xi|^{p}$, we summarize our computations as follows:

$$
\begin{aligned}
\mathbb{E} \int_{M} & S_{m}\left(u^{\varepsilon}(t)\right) d V_{h}(x) \leq \mathbb{E}\left\|u_{0}^{\varepsilon}\right\|_{L^{p}(M)}^{p}+C\left(1+\mathbb{E}\left\|u_{0}^{\varepsilon}\right\|_{L^{p}(M)}^{p}\right) \\
& +D_{1} p(p-1) \frac{t}{2}+\int_{0}^{t} D_{1} p(p-1) \mathbb{E} \int_{M} S_{m}\left(u^{\varepsilon}(s)\right) d V_{h}(x) d s .
\end{aligned}
$$

As in the proof of Proposition 7.4, we apply the Gronwall inequality to obtain

$$
\mathbb{E} \int_{M} S_{m}\left(u^{\varepsilon}(t)\right) d V_{h}(x) \leq C\left(1+\mathbb{E}\left\|u_{0}^{\varepsilon}\right\|_{L^{p}(M)}^{p}\right), \quad t \in[0, T],
$$

and hence, by Fatou's lemma,

$$
\mathbb{E}\left\|u^{\varepsilon}(t)\right\|_{L^{p}(M)}^{p} \leq C\left(1+\mathbb{E}\left\|u_{0}^{\varepsilon}\right\|_{L^{p}(M)}^{p}\right) .
$$

Let us return to 7.5 . Taking into account the previous computations,

$$
\begin{gathered}
\int_{M} S_{m}\left(u^{\varepsilon}(t)\right) d V_{h}(x)+\varepsilon \int_{0}^{t} \int_{M}\left|\nabla u^{\varepsilon}(s)\right|_{h}^{2} S_{m}^{\prime \prime}\left(u^{\varepsilon}(s)\right) d V_{h}(x) d s \\
\leq\left\|u_{0}^{\varepsilon}\right\|_{L^{p}(M)}^{p}+\sum_{k \geq 1} \int_{0}^{t} \int_{M} S_{m}^{\prime}\left(u^{\varepsilon}(s)\right) g_{k}\left(x, u^{\varepsilon}(s)\right) d V_{h}(x) d \beta_{k}(s) \\
+D_{1} p(p-1) \frac{t}{2}+\int_{0}^{t} D_{1} p(p-1)\left\|u^{\varepsilon}(s)\right\|_{L^{p}(M)}^{p} d s
\end{gathered}
$$

$\mathbb{P}$-a.s., for any $t \in[0, T]$. Note that $S_{m}$ is convex and, for any $\xi \in \mathbb{R}, S_{m}(\xi) \rightarrow|\xi|^{p}$ and $S_{m}^{\prime \prime}(\xi) \rightarrow p(p-1)|\xi|^{p-2}$ as $m \rightarrow \infty$. As a result of this, the superadditivity of liminf, Fatou's lemma, and taking the supremum over $[0, T]$, we obtain

$$
\begin{aligned}
& \sup _{0 \leq t \leq T}\left\|u^{\varepsilon}(t)\right\|_{L^{p}(M)}^{p}+\varepsilon p(p-1) \int_{0}^{T} \int_{M}\left|u^{\varepsilon}(s)\right|^{p-2}\left|\nabla u^{\varepsilon}(s)\right|_{h}^{2} d V_{h}(x) d s \\
& \quad \leq 2\left\|u_{0}^{\varepsilon}\right\|_{L^{p}(M)}^{p}+D_{1} p(p-1) T+\int_{0}^{T} 2 D_{1} p(p-1)\left\|u^{\varepsilon}(s)\right\|_{L^{p}(M)}^{p} d s+\mathcal{I},
\end{aligned}
$$

$\mathbb{P}$-a.s., for any $t \in[0, T]$, where

$$
\mathcal{I}:=2 \liminf _{m \rightarrow \infty} \sup _{0 \leq t \leq T}\left|\sum_{k \geq 1} \int_{0}^{t} \int_{M} S_{m}^{\prime}\left(u^{\varepsilon}(s)\right) g_{k}\left(x, u^{\varepsilon}(s)\right) d V_{h}(x) d \beta_{k}(s)\right| .
$$

Note that $u^{\varepsilon}$ admits an $L^{r}(M, h)$-continuous modification for any $r \in(2, p)$, and thus for $p$ as well. This is a consequence of the fact that this is already known for $p=2$, the $L^{p}$ estimate 7.6 , and a standard interpolation argument.

Using the Fatou lemma and arguing as we have done several times before,

$$
\mathbb{E} \mathcal{I} \leq C\left(1+\mathbb{E}\left\|u_{0}^{\varepsilon}\right\|_{L^{p}(M)}^{p}\right)
$$

Taking the expectation in $(7.7)$, noting that $\varepsilon p(p-1)>\varepsilon$, we thus obtain

$$
\begin{aligned}
& \mathbb{E} \sup _{0 \leq t \leq T}\left\|u^{\varepsilon}(t)\right\|_{L^{p}(M)}^{p}+\varepsilon \mathbb{E} \int_{0}^{T} \int_{M}\left|u^{\varepsilon}(s)\right|^{p-2}\left|\nabla u^{\varepsilon}(s)\right|_{h}^{2} d V_{h}(x) d s \\
& \leq 2 \mathbb{E}\left\|u_{0}^{\varepsilon}\right\|_{L^{p}(M)}^{p}+D_{1} p(p-1) T+\int_{0}^{T} 2 D_{1} p(p-1) \mathbb{E}\left\|u^{\varepsilon}(s)\right\|_{L^{p}(M)}^{p} d s \\
& \quad+C\left(1+\mathbb{E}\left\|u_{0}^{\varepsilon}\right\|_{L^{p}(M)}^{p}\right) .
\end{aligned}
$$

Thanks to (7.6), we conclude that there is a constant $K_{p}>0$, depending on $D_{1}$ and $T$ but independent of $\varepsilon, C_{0}, r, L, C_{1}, D_{2}$, such that $(7.3)$ holds. 
7.3. Bounds on kinetic measure. In view of Propositions 7.4 and 7.5 , there is an $\varepsilon$-independent constant $C_{p}$ such that

$$
\mathbb{E} \int_{[0, T] \times M \times \mathbb{R}}|\xi|^{p} m^{\varepsilon}(d s, d x, d \xi) \leq C_{p}, \quad p \in[2, \infty),
$$

where the "parabolic" kinetic measure $m^{\varepsilon}$ is defined in Proposition 7.1

We also have the improved estimate

$$
\left.\left.\mathbb{E}\left|\int_{[0, T] \times M \times \mathbb{R}}\right| \xi\right|^{2 p} m^{\varepsilon}(d s, d x, d \xi)\right|^{2} \leq C_{p}, \quad p \in[0, \infty),
$$

To derive this estimate we replicate the proof of Proposition 7.5 , this time with $S_{m}(\xi) \rightarrow|\xi|^{q}$ as $m \rightarrow \infty$, where $q:=2 p+2$. Indeed, we obtain

$$
\begin{aligned}
& \int_{0}^{T} \int_{M} \varepsilon\left|\nabla u^{\varepsilon}(s)\right|_{h}^{2} S_{m}^{\prime \prime}\left(u^{\varepsilon}(s)\right) d V_{h}(x) d s \\
& \leq\left\|u_{0}\right\|_{L^{q}(M)}^{q}+\sum_{k \geq 1} \int_{0}^{T} \int_{M} S_{m}^{\prime}\left(u^{\varepsilon}(s)\right) g_{k}\left(x, u^{\varepsilon}(s)\right) d V_{h}(x) d \beta_{k}(s) \\
& \quad+C_{q}\left(1+\int_{0}^{T}\left\|u^{\varepsilon}(s)\right\|_{L^{q}(M)}^{q} d s\right), \quad \text { P-almost surely }
\end{aligned}
$$

We square and then take the expectation. By the Itô isometry,

$$
\begin{aligned}
& \mathcal{D}(m):=\left.\left.\mathbb{E}\left|\int_{0}^{T} \int_{M} \varepsilon\right| \nabla u^{\varepsilon}(s)\right|_{h} ^{2} S_{m}^{\prime \prime}\left(u^{\varepsilon}(s)\right) d V_{h}(x) d s\right|^{2} \\
& \leq C+C \mathbb{E}\left\|u_{0}\right\|_{L^{q}(M)}+C \mathbb{E} \int_{0}^{T} \sum_{k \geq 1}\left|\int_{M} S_{m}^{\prime}\left(u^{\varepsilon}(s)\right) g_{k}\left(x, u^{\varepsilon}(s)\right) d V_{h}(x)\right|^{2} d s \\
&+C \mathbb{E}\left|\int_{0}^{T}\left\|u^{\varepsilon}(s)\right\|_{L^{q}(M)}^{q} d s\right|^{2},
\end{aligned}
$$

where the constant $C$ is independent of $\varepsilon$. Making use of $\|\cdot\|_{L^{q}(M)} \leq\|\cdot\|_{L^{2 q}(M)}$ and Proposition 7.5, we arrive at

$$
\mathcal{D}(m) \leq C+C \mathbb{E} \int_{0}^{T} \sum_{k \geq 1}\left|\int_{M} S_{m}^{\prime}\left(u^{\varepsilon}(s)\right) g_{k}\left(x, u^{\varepsilon}(s)\right) d V_{h}(x)\right|^{2} d s .
$$

for a new constant $C$. By Jensen's inequality, elementary properties of the function $S_{m}$, cf. 7.4, 2.3, and again Proposition 7.5, we can bound the second term on the right-hand side by a constant. The final result is

$$
\mathcal{D}(m) \leq C_{q},
$$

for some positive constant $C_{q}$ independent of $\varepsilon$ (and $m$ ).

Seeing that $0 \leq S_{m}^{\prime \prime}(\xi) \uparrow q(q-1)|\xi|^{q-2}$ pointwise as $m \rightarrow \infty$, the monotone convergence theorem implies

$$
\lim _{m \rightarrow \infty} \mathcal{D}(m)=\mathcal{D}, \quad \mathbb{P} \text {-almost surely, }
$$

where $\mathcal{D}=\mathcal{D}(\omega) \in[0, \infty]$ is defined by

$$
\mathcal{D}:=\left.\left.\left|\int_{0}^{T} \int_{M} \varepsilon\right| \nabla u^{\varepsilon}(s)\right|_{h} ^{2} q(q-1)\left|u^{\varepsilon}(s)\right|^{q-2} d V_{h}(x) d s\right|^{2} .
$$


On the other hand, since $q=2 p+2 \geq 2$, Propositions 7.4 and 7.5 ensure that $\mathcal{D}<\infty$ (P-a.s.). In particular, $\liminf _{m \rightarrow \infty} \mathcal{D}(m)=\mathcal{D} \in[0, \infty)$ (P्P-a.s.). By means of Fatou's Lemma and $(7.9), \mathbb{E} \mathcal{D} \leq C_{q}$, that is,

$$
\left.\left.\mathbb{E}\left|\int_{0}^{T} \int_{M} \varepsilon\right| \nabla u^{\varepsilon}(s)\right|_{h} ^{2}\left|u^{\varepsilon}(s)\right|^{2 p} d V_{h}(x) d s\right|^{2} \leq C_{p}, \quad p \in[0, \infty) .
$$

The claim (7.8) follows from this.

7.4. Existence part of Theorem 3.2. Following [15, Section 4] closely, using the a priori bounds derived in the preceding paragraphs, $\rho^{\varepsilon}=\mathbb{I}_{u^{\varepsilon}>\xi}$ converges weakly- $\star$ to some $\rho$ in $L^{\infty}(\Omega \times(0, T) \times M \times \mathbb{R})$, along a subsequence as $\varepsilon \rightarrow 0$ (not relabeled). Moreover, $m^{\varepsilon}$ converges weakly-^ to some $m$ in $L^{2}(\Omega ; \mathcal{M})$, where $\mathcal{M}$ denotes the space of bounded Borel measures on $(0, T) \times M \times \mathbb{R}$. The limit $(\rho, m)$ constitutes a generalized kinetic solution according to Definition 3.3. Thanks to Proposition 4.1. $\rho$ is actually a kinetic function, that is, $\rho=\mathbb{I}_{u>\xi}$ for some $u$. The function $u$ is a kinetic solution according to Definition 3.2. Moreover, $u^{\varepsilon}=\int_{\mathbb{R}}\left(\rho^{\varepsilon}-\mathbb{I}_{0>\xi}\right) d \xi$ converges strongly to $u=\int_{\mathbb{R}}\left(\rho-\mathbb{I}_{0>\xi}\right) d \xi$ in $L^{p}(\Omega \times[0, T] \times M)$ :

$$
\lim _{\varepsilon \rightarrow 0} \mathbb{E}\left\|u^{\varepsilon}-u\right\|_{L^{p}([0, T] \times M)}^{p}=0, \quad \forall p \in[1, \infty) .
$$

Since the kinetic solution $u$ is unique, cf. Theorem 3.2 , the entire sequence converges. For the details, we refer again to 15 .

\section{APpEndix}

Lemma 8.1. Let $F \in C_{b}^{0}(\mathbb{R})$. Suppose $\left(u_{j}\right)_{j} \subset L^{2}(M, h)$ converges to $u$ in $L^{2}(M, h)$. Then $F\left(u_{j}\right) \rightarrow F(u)$ for $j \rightarrow \infty$ in $L^{p}(M, h)$, for any $p \in[1, \infty[$.

Proof. We pick any subsequence $\left(j_{k}\right)_{k}$. Then the reverse dominated convergence theorem guarantees that there exists $\left(j_{k_{l}}\right)_{l}$ such that we have pointwise convergence for a.e. $x \in M$. Therefore, by continuity of $F$, we obtain

$$
\left|F\left(u_{j_{k_{l}}}(x)\right)-F(u(x))\right|^{p} \rightarrow 0, \quad \text { as } l \rightarrow \infty,
$$

for a.e. $x \in M$. Moreover, $\left|F\left(u_{j_{k_{l}}}(x)\right)-F(u(x))\right|^{p} \leq 2^{p}\|F\|_{L^{\infty}}^{p}$, and thus by dominated convergence $F\left(u_{j_{k}}\right)$ tends in $L^{p}(M, h)$ to $F(u)$ as $l \rightarrow \infty$. Since $\left(j_{k}\right)_{k}$ was arbitrary, the same result must hold for the whole sequence.

Lemma 8.2. Let $\psi$ be in $\mathcal{D}(\mathbb{R})$ and $\psi \geq 0$. Let $\mu$ and $\nu$ be finite Borel measures on $\mathbb{R}$. Let $\eta$ be a standard mollifier on $\mathbb{R}$ and set $\eta_{\varepsilon}(\cdot):=\varepsilon^{-1} \eta(\dot{\bar{\varepsilon}})$ for $\varepsilon$ positive. Then

$$
\int_{\mathbb{R}} \psi(u) \int_{\mathbb{R}^{2}}|\xi-\gamma|^{2} \eta_{\varepsilon}(u-\xi) \eta_{\varepsilon}(u-\gamma) \mu \otimes \nu(d \xi, d \gamma) d u \leq C \varepsilon
$$

where $C=4\|\psi\|_{L^{\infty}}\|\eta\|_{L^{\infty}}(\mu \otimes \nu)\left(\mathbb{R}^{2}\right)$ 
Proof. By direct computation, setting $S_{y}:=B_{1}(0) \cap B_{1}(y)$ for $y \in \mathbb{R}$, we see that

$$
\begin{aligned}
& \int_{\mathbb{R}} \psi(u) \int_{\mathbb{R}^{2}}|\xi-\gamma|^{2} \eta_{\varepsilon}(u-\xi) \eta_{\varepsilon}(u-\gamma) \mu \otimes \nu(d \xi, d \gamma) d u \\
& =\int_{\mathbb{R}^{2}}|\xi-\gamma|^{2}\left[\int_{\mathbb{R}} \psi(u) \eta_{\varepsilon}(u-\xi) \eta_{\varepsilon}(u-\gamma) d u\right] \mu \otimes \nu(d \xi, d \gamma) \\
& =\int_{\mathbb{R}^{2}}|\xi-\gamma|^{2}\left[\int_{S_{\frac{\gamma-\xi}{\varepsilon}}} \psi(\varepsilon v+\xi) \eta(v) \varepsilon^{-1} \eta\left(v+\frac{\xi-\gamma}{\varepsilon}\right) d v\right] \mu \otimes \nu(d \xi, d \gamma) \\
& =\int_{|\gamma-\xi| \leq 2 \varepsilon}|\xi-\gamma|^{2}\left[\int_{S_{\frac{\gamma-\xi}{\varepsilon}}} \psi(\varepsilon v+\xi) \eta(v) \varepsilon^{-1} \eta\left(v+\frac{\xi-\gamma}{\varepsilon}\right) d v\right] \mu \otimes \nu(d \xi, d \gamma) \\
& \leq\|\psi\|_{L^{\infty}}\|\eta\|_{L^{\infty}} \varepsilon^{-1} \int_{|\gamma-\xi| \leq 2 \varepsilon}|\xi-\gamma|^{2} \mu \otimes \nu(d \xi, d \gamma) \leq C \varepsilon
\end{aligned}
$$

\section{REFERENCES}

[1] P. Amorim, M. Ben-Artzi, and P. G. LeFloch. Hyperbolic conservation laws on manifolds: total variation estimates and the finite volume method. Methods Appl. Anal., 12(3):291-323, 2005.

[2] P. Amorim, P. G. LeFloch, and B. Okutmustur. Finite volume schemes on Lorentzian manifolds. Commun. Math. Sci., 6(4):1059-1086, 2008.

[3] T. Aubin. Some nonlinear problems in Riemannian geometry. Springer Monographs in Mathematics. Springer-Verlag, Berlin, 1998.

[4] D. Bakry. étude des transformations de Riesz dans les variétés riemanniennes à courbure de Ricci minorée. In Séminaire de Probabilités, XXI, volume 1247 of Lecture Notes in Math., pages 137-172. Springer, Berlin, 1987.

[5] C. Bauzet, G. Vallet, and P. Wittbold. The Cauchy problem for conservation laws with a multiplicative stochastic perturbation. J. Hyperbolic Differ. Equ., 9(4):661-709, 2012.

[6] A. Beljadid and P. G. LeFloch. A central-upwind geometry-preserving method for hyperbolic conservation laws on the sphere. Commun. Appl. Math. Comput. Sci., 12(1):81-107, 2017.

[7] M. Ben-Artzi and P. G. LeFloch. Well-posedness theory for geometry-compatible hyperbolic conservation laws on manifolds. Ann. Inst. H. Poincaré Anal. Non Linéaire, 24(6):989-1008, 2007.

[8] M. Ben-Artzi, J. Falcovitz, and P. G. LeFloch. Hyperbolic conservation laws on the sphere. A geometry-compatible finite volume scheme. J. Comput. Phys., 228(16):5650-5668, 2009.

[9] I. H. Biswas, K. H. Karlsen, and A. K. Majee. Conservation laws driven by Lévy white noise. J. Hyperbolic Differ. Equ., 12(3):581-654, 2015.

[10] G.-Q. Chen, Q. Ding, and K. H. Karlsen. On nonlinear stochastic balance laws. Arch. Ration. Mech. Anal., 204(3):707-743, 2012.

[11] C. M. Dafermos. Hyperbolic conservation laws in continuum physics, volume 325 of Grundlehren der Mathematischen Wissenschaften [Fundamental Principles of Mathematical Sciences]. Springer-Verlag, Berlin, fourth edition, 2016.

[12] A.-L. Dalibard. Kinetic formulation for heterogeneous scalar conservation laws. Ann. Inst. H. Poincaré Anal. Non Linéaire, 23(4):475-498, 2006.

[13] G. Da Prato and J. Zabczyk. Stochastic equations in infinite dimensions, volume 152 of Encyclopedia of Mathematics and its Applications. Cambridge University Press, Cambridge, second edition, 2014.

[14] E. B. Davies. Pointwise bounds on the space and time derivatives of heat kernels. J. Operator Theory, 21(2):367-378, 1989.

[15] A. Debussche and J. Vovelle. Scalar conservation laws with stochastic forcing. J. Funct. Anal., 259(4):1014-1042, 2010.

[16] A. Debussche and J. Vovelle. Invariant measure of scalar first-order conservation laws with stochastic forcing. Probab. Theory Related Fields, 163(3-4):575-611, 2015.

[17] A. Debussche, M. Hofmanová, and J. Vovelle. Degenerate parabolic stochastic partial differential equations: Quasilinear case. Ann. Probab., 44(3):1916-1955, 2016.

[18] L. Denis, A. Matoussi, and L. Stoica. $L^{p}$ estimates for the uniform norm of solutions of quasilinear SPDE's. Probab. Theory Related Fields, 133(4):437-463, 2005. 
[19] G. de Rham. Differentiable manifolds, volume 266 of Grundlehren der Mathematischen Wissenschaften. Springer-Verlag, Berlin, 1984.

[20] J. Diestel and J. J. Uhl, Jr. Vector measures. American Mathematical Society, Providence, R.I., 1977. With a foreword by B. J. Pettis, Mathematical Surveys, No. 15.

[21] R. J. DiPerna and P.-L. Lions. Ordinary differential equations, transport theory and Sobolev spaces. Invent. Math., 98(3):511-547, 1989.

[22] S. Dotti and J. Vovelle. Convergence of Approximations to Stochastic Scalar Conservation Laws. Arch. Ration. Mech. Anal., 230(2):539-591, 2018.

[23] S. Dotti and J. Vovelle. Convergence of the Finite Volume Method for scalar conservation laws with multiplicative noise: an approach by kinetic formulation. ArXiv e-prints, Nov. 2016.

[24] G. Dziuk, D. Kröner, and T. Müller. Scalar conservation laws on moving hypersurfaces. Interfaces Free Bound., 15(2):203-236, 2013.

[25] W. E, K. Khanin, A. Mazel, and Y. Sinai. Invariant measures for Burgers equation with stochastic forcing. Ann. of Math. (2), 151(3):877-960, 2000.

[26] D. Elworthy. Geometric aspects of diffusions on manifolds. In École d'Été de Probabilités de Saint-Flour XV-XVII, 1985-87, volume 1362 of Lecture Notes in Math., pages 277-425. Springer, Berlin, 1988.

[27] J. Feng and D. Nualart. Stochastic scalar conservation laws. J. Funct. Anal., 255(2):313-373, 2008.

[28] B. Gess and P. E. Souganidis. Scalar conservation laws with multiple rough fluxes. Commun. Math. Sci., 13(6):1569-1597, 2015.

[29] B. Gess and P. E. Souganidis. Long-time behavior, invariant measures, and regularizing effects for stochastic scalar conservation laws. Comm. Pure Appl. Math., 70(8):1562-1597, 2017.

[30] J. Giesselmann. A convergence result for finite volume schemes on Riemannian manifolds. M2AN Math. Model. Numer. Anal., 43(5):929-955, 2009.

[31] J. Giesselmann and T. Müller. Geometric error of finite volume schemes for conservation laws on evolving surfaces. Numer. Math., 128(3):489-516, 2014.

[32] M. Graf, M. Kunzinger, and D. Mitrovic. Well-posedness theory for degenerate parabolic equations on Riemannian manifolds. J. Differential Equations, 263:4787-4825, Oct. 2017.

[33] A. Grigor'yan. Heat kernel and analysis on manifolds, volume 47 of AMS/IP Studies in Advanced Mathematics. American Mathematical Society, 2009.

[34] E. Hebey. Nonlinear analysis on manifolds: Sobolev spaces and inequalities, volume 5 of Courant Lecture Notes in Mathematics. American Mathematical Society, 1999.

[35] M. Hofmanová. Degenerate parabolic stochastic partial differential equations. Stochastic Process. Appl., 123(12):4294-4336, 2013.

[36] K. H. Karlsen and E. B. Storrøsten. On stochastic conservation laws and Malliavin calculus. J. Funct. Anal., 272:421-497, 2017.

[37] K. H. Karlsen and E. B. Storrøsten. Analysis of a splitting method for stochastic balance laws. IMA J. Numer. Anal., 38(1):1-56, 2018.

[38] J. U. Kim. On a stochastic scalar conservation law. Indiana Univ. Math. J., 52(1):227-256, 2003.

[39] K. Kobayasi and D. Noboriguchi. A stochastic conservation law with nonhomogeneous Dirichlet boundary conditions. Acta Math. Vietnam., 41(4):607-632, 2016.

[40] D. Kröner, T. Müller, and L. M. Strehlau. Traces for functions of bounded variation on manifolds with applications to conservation laws on manifolds with boundary. SIAM J. Math. Anal., 47(5):3944-3962, 2015.

[41] N. V. Krylov. A relatively short proof of Itô's formula for SPDEs and its applications. Stochastic Partial Differential Equations: Analysis and Computations, 1(1):152-174, 2013.

[42] N. Krylov and B. Rozovskii. Stochastic evolution equations. Journal of Mathematical Sciences, 16(4):1233-1277, 71981.

[43] J. M. Lee. Introduction to smooth manifolds, volume 218 of Graduate Texts in Mathematics. Springer-Verlag, New York, 2003.

[44] P. G. LeFloch and H. Makhlof. A geometry-preserving finite volume method for compressible fluids on Schwarzschild spacetime. Commun. Comput. Phys., 15(3):827-852, 2014.

[45] P. G. LeFloch and B. Okutmustur. Hyperbolic conservation laws on spacetimes. A finite volume scheme based on differential forms. Far East J. Math. Sci. (FJMS), 31(1):49-83, 2008.

[46] P. G. LeFloch, B. Okutmustur, and W. Neves. Hyperbolic conservation laws on manifolds. An error estimate for finite volume schemes. Acta Math. Sin. (Engl. Ser.), 25(7):1041-1066, 2009.

[47] D. Lengeler and T. Müller. Scalar conservation laws on constant and time-dependent Riemannian manifolds. J. Differential Equations, 254(4):1705-1727, 2013. 
[48] G. Lv and J.-L. Wu. Renormalized entropy solutions of stochastic scalar conservation laws with boundary condition. J. Funct. Anal., 271(8):2308-2338, 2016.

[49] P.-L. Lions, B. Perthame, and P. E. Souganidis. Stochastic averaging lemmas for kinetic equations. In Séminaire Laurent Schwartz-Équations aux dérivées partielles et applications. Année 2011-2012, Sémin. Équ. Dériv. Partielles, pages Exp. No. XXVI, 17. École Polytech., Palaiseau, 2013.

[50] P.-L. Lions, B. Perthame, and P. Souganidis. Scalar conservation laws with rough (stochastic) fluxes. Stoch. Partial Differ. Equ. Anal. Comput., 1(4):664-686, 2013.

[51] P.-L. Lions, B. Perthame, and P. E. Souganidis. Scalar conservation laws with rough (stochastic) fluxes: the spatially dependent case. Stoch. Partial Differ. Equ. Anal. Comput., 2(4):517-538, 2014.

[52] E. Y. Panov. On the Cauchy problem for a first-order quasilinear equation on a manifold. Differ. Uravn., 33(2):257-266, 287, 1997.

[53] E. Y. Panov. On the Dirichlet problem for first order quasilinear equations on a manifold. Trans. Amer. Math. Soc., 363(5):2393-2446, 2011.

[54] B. Perthame. Kinetic formulation of conservation laws, volume 21 of Oxford Lecture Series in Mathematics and its Applications. Oxford University Press, Oxford, 2002.

[55] C. Prévôt and M. Röckner. A concise course on stochastic partial differential equations, volume 1905 of Lecture Notes in Mathematics. Springer, Berlin, 2007.

[56] P. Protter. Stochastic integration and differential equations, volume 21 of Applications of Mathematics (New York). Springer-Verlag, Berlin, 1990.

[57] R. S. Strichartz. Analysis of the Laplacian on the complete Riemannian manifold. J. Funct. Anal., 52(1):48-79, 1983.

[58] G. Vallet and P. Wittbold. On a stochastic first-order hyperbolic equation in a bounded domain. Infin. Dimens. Anal. Quantum Probab. Relat. Top., 12(4):613-651, 2009.

(Luca Galimberti)

DEPARTMENT OF MATHEMATICS

UNIVERSITY OF OSLO

P.O. Box 1053, BLINDERN

N-0316 Oslo, Norway

E-mail address: lucaga@math.uio.no

(Kenneth Hvistendahl Karlsen)

DEPARTMENT OF MATHEMATICS

UNIVERSITY OF OSLO

P.O. Box 1053, Blindern

N-0316 Oslo, Norway

E-mail address: kennethkarlsen@me.com 